\title{
Hybrid Extragradient Methods for Finding Zeros of Accretive Operators and Solving Variational Inequality and Fixed Point Problems in Banach Spaces
}

\author{
Lu-Chuan Ceng ${ }^{1,2}$ and Ching-Feng Wen ${ }^{3}$ \\ ${ }^{1}$ Department of Mathematics, Shanghai Normal University, Shanghai 200234, China \\ ${ }^{2}$ Scientific Computing Key Laboratory of Shanghai Universities, Shanghai 200234, China \\ ${ }^{3}$ Center for Fundamental Science, Kaohsiung Medical University, Kaohsiung 807, Taiwan \\ Correspondence should be addressed to Ching-Feng Wen; cfwen@kmu.edu.tw
}

Received 8 July 2013; Accepted 11 August 2013

Academic Editor: Jen-Chih Yao

Copyright (C) 2013 L.-C. Ceng and C.-F. Wen. This is an open access article distributed under the Creative Commons Attribution License, which permits unrestricted use, distribution, and reproduction in any medium, provided the original work is properly cited.

\begin{abstract}
We introduce and analyze hybrid implicit and explicit extragradient methods for finding a zero of an accretive operator and solving a general system of variational inequalities and a fixed point problem of an infinite family of nonexpansive self-mappings in a uniformly convex Banach space $X$ which has a uniformly Gateaux differentiable norm. We establish some strong convergence theorems for hybrid implicit and explicit extra-gradient algorithms under suitable assumptions. Furthermore, we derive the strong convergence of hybrid implicit and explicit extragradient algorithms for finding a common element of the set of zeros of an accretive operator and the common fixed point set of an infinite family of nonexpansive self-mappings and a self-mapping whose complement is strictly pseudocontractive and strongly accretive in $X$. The results presented in this paper improve, extend, supplement, and develop the corresponding results announced in the earlier and very recent literature.
\end{abstract}

\section{Introduction}

Let $X$ be a real Banach space whose dual space is denoted by $X^{*}$. Let $U=\{x \in X:\|x\|=1\}$ denote the unite sphere of $X$. A Banach space $X$ is said to be uniformly convex if, for each $\epsilon \in(0,2]$, there exists $\delta>0$ such that for all $x, y \in U$,

$$
\|x-y\| \geq \epsilon \Longrightarrow \frac{\|x+y\|}{2} \leq 1-\delta .
$$

It is known that a uniformly convex Banach space is reflexive and strict convex. The normalized duality mapping $J: X \rightarrow$ $2^{X^{*}}$ is defined by

$$
J(x)=\left\{x^{*} \in X^{*}:\left\langle x, x^{*}\right\rangle=\|x\|^{2}=\left\|x^{*}\right\|^{2}\right\}, \quad \forall x \in X,
$$

where $\langle\cdot, \cdot\rangle$ denotes the generalized duality pairing. It is an immediate consequence of the Hahn-Banach theorem that $J(x)$ is nonempty for each $x \in X$. Moreover, it is known that $J$ is single-valued if and only if $X$ is smooth, whereas if $X$ is uniformly smooth, then $J$ is norm-to-norm uniformly continuous on bounded subsets of $X$. If $X$ has a uniformly Gateaux differentiable norm, then the duality mapping $J$ is norm-to-weak ${ }^{*}$ uniformly continuous on bounded subsets of $X$; see for example [1].

Let $C$ be a nonempty closed convex subset of a real Banach space $X$. A mapping $T: C \rightarrow C$ is called nonexpansive if

$$
\|T x-T y\| \leq\|x-y\|, \quad \forall x, y \in C .
$$

The set of fixed points of $T$ is denoted by $\operatorname{Fix}(T)$. We use the notation $\rightarrow$ to indicate the weak convergence and $\rightarrow$ to indicate the strong convergence.

Recall that (possibly multivalued) operator $A \subset E \times E$ with domain $D(A)$ and range $R(A)$ in $E$ is accretive if, for each $x_{i} \in D(A)$ and $y_{i} \in A x_{i}(i=1,2)$, there exists a $j\left(x_{1}-x_{2}\right) \in$ $J\left(x_{1}-x_{2}\right)$ such that $\left\langle y_{1}-y_{2},\left(x_{1}-x_{2}\right)\right\rangle \geq 0$ (here $J$ is the duality mapping). An accretive operator $A$ is said to satisfy the range condition if $\overline{D(A)} \subset R(I+r A)$ for all $r>0$. An accretive 
operator $A$ is $m$-accretive if $R(I+r A)=E$ for each $r>0$. If $A$ is an accretive operator which satisfies the range condition, then we can define, for each $r>0$, a mapping $J_{r}: R(I+r A) \rightarrow$ $D(A)$ defined by $J_{r}=(I+r A)^{-1}$, which is called the resolvent of $A$. We know that $J_{r}$ is nonexpansive and $\operatorname{Fix}\left(J_{r}\right)=A^{-1} 0$ for all $r>0$. Hence

$$
\operatorname{Fix}\left(J_{r}\right)=A^{-1} 0=\{z \in D(A): 0 \in A z\}
$$

If $A^{-1} 0 \neq \emptyset$, then the inclusion $0 \in A z$ is solvable.

The following resolvent identity is well known to us; see [2], where more details on accretive operators can be found.

Proposition 1 (resolvent identity). For $\lambda>0, \mu>0$, and $x \in$ $X$,

$$
J_{\lambda} x=J_{\mu}\left(\frac{\mu}{\lambda} x+\left(1-\frac{\mu}{\lambda}\right) J_{\lambda} x\right) .
$$

Recently, Aoyama et al. [3] studied the following iterative scheme in a uniformly convex Banach space having a uniformly Gareaux differentiable norm: for resolvents $J_{r_{n}}$ of an accretive operator $A$ such that $A^{-1} 0 \neq \emptyset$ and $\overline{D(A)} \subset C \subset$ $\bigcap_{r>0} R(I+r A)$ and $\left\{\alpha_{n}\right\} \subset(0,1)$,

$$
\begin{gathered}
x_{0}=x \in C, \\
x_{n+1}=\alpha_{n} x+\left(1-\alpha_{n}\right) J_{r_{n}} x_{n} .
\end{gathered}
$$

They proved that the sequence $\left\{x_{n}\right\}$ generated by (6) converges strongly to a zero of $A$ under appropriate assumptions on $\left\{\alpha_{n}\right\}$ and $\left\{r_{n}\right\}$. Subsequently, Ceng et al. [4] introduced and analyzed the following composite iterative scheme in either a uniformly smooth Banach space or a reflexive Banach space having a weakly sequentially continuous duality mapping,

$$
\begin{gathered}
x_{0}=x \in E, \\
y_{n}=\alpha_{n} u+\left(1-\alpha_{n}\right) J_{r_{n}} x_{n}, \\
x_{n+1}=\left(1-\beta_{n}\right) y_{n}+\beta_{n} J_{r_{n}} y_{n},
\end{gathered}
$$

where $u \in \overline{D(A)}$ is an arbitrary (but fixed) element, under the following control conditions:

(H1) $\lim _{n \rightarrow \infty} \alpha_{n}=0$;

(H2) $\sum_{n=0}^{\infty} \alpha_{n}=\infty$, or, equivalently, $\prod_{n=0}^{\infty}\left(1-\alpha_{n}\right)=0$;

(H3) $\sum_{n=1}^{\infty}\left|\alpha_{n}-\alpha_{n-1}\right|<\infty$;

(H4) $r_{n} \geq \varepsilon$, for all $n \geq 0$, for some $\varepsilon>0$ and $\sum_{n=1}^{\infty} \mid r_{n}-$ $r_{n-1} \mid<\infty$;

(H5) $\beta_{n} \in[0, a)$ for some $a \in(0,1)$ and $\sum_{n=1}^{\infty}\left|\beta_{n}-\beta_{n-1}\right|<$ $\infty$.

Furthermore, as the viscosity approximation method, Jung [5] purposed and analyzed the following composite iterative scheme for finding a zero of an accretive operator $A$ : for resolvent $J_{r_{n}}$ of an accretive operator $A$ such that $A^{-1} 0 \neq \emptyset$ and $\overline{D(A)} \subset C \subset \bigcap_{r>0} R(I+r A), f \in \Xi_{C}\left(\Xi_{C}\right.$ denotes the set of all contractions on $C)$ and $\left\{\alpha_{n}\right\},\left\{\beta_{n}\right\} \subset(0,1)$,

$$
\begin{gathered}
x_{0}=x \in C, \\
y_{n}=\alpha_{n} f\left(x_{n}\right)+\left(1-\alpha_{n}\right) J_{r_{n}} x_{n}, \\
x_{n+1}=\left(1-\beta_{n}\right) y_{n}+\beta_{n} J_{r_{n}} y_{n} .
\end{gathered}
$$

He established the strong convergence of the sequence $\left\{x_{n}\right\}$ generated by (8) to a zero of $A$ under certain appropriate conditions.

Theorem 2 (see [5, Theorem 3.1]). Let $X$ be a strictly convex and reflexive Banach space having a uniformly Gateaux differentiable norm. Let $C$ be a nonempty closed convex subset of $X$ and $A \subset X \times X$ an accretive operator in $X$ such that $A^{-1} 0 \neq \emptyset$ and $\overline{D(A)} \subset C \subset \bigcap_{r>0} R(I+r A)$. Let $\left\{\alpha_{n}\right\}$ and $\left\{\beta_{n}\right\}$ be sequences in $(0,1)$ which satisfy the following conditions:
(C1) $\lim _{n \rightarrow \infty} \alpha_{n}=0$ and $\sum_{n=0}^{\infty} \alpha_{n}=\infty$;
(B1) $\beta_{n} \in[0, a)$ for some $0<a<1$ for all $n \geq 0$.

Let $f \in \Xi_{C}$ and $x_{0} \in C$ be chosen arbitrarily. Let $\left\{x_{n}\right\}$ be a sequence generated by (8) for $r_{n}>0$. If $\left\{x_{n}\right\}$ is asymptotically regular, that is, $\lim _{n \rightarrow \infty}\left\|x_{n+1}-x_{n}\right\|=0$, then $\left\{x_{n}\right\}$ converges strongly to $q \in A^{-1} 0$, which is the unique solution of the variational inequality problem (VIP)

$$
\langle(I-f) q, J(q-p)\rangle \leq 0, \quad \forall f \in \Xi_{C}, p \in A^{-1} 0 .
$$

On the other hand, we first recall the following concepts.

Definition 3. Let $C$ be a nonempty closed convex subset of a real Banach space $X$ and let $A: C \rightarrow X$ be a mapping of $C$ into $X$. Then $A$ is said to be:

(i) accretive if, for each $x, y \in C$, there exists $j(x-y) \in$ $J(x-y)$ such that

$$
\langle A x-A y, j(x-y)\rangle \geq 0,
$$

where $J$ is the normalized duality mapping;

(ii) $\alpha$-strongly accretive if, for each $x, y \in C$, there exists $j(x-y) \in J(x-y)$ such that

$$
\langle A x-A y, j(x-y)\rangle \geq \alpha\|x-y\|^{2},
$$

for some $\alpha \in(0,1)$;

(iii) $\beta$-inverse-strongly-accretive if, for each $x, y \in C$, there exists $j(x-y) \in J(x-y)$ such that

$$
\langle A x-A y, j(x-y)\rangle \geq \beta\|A x-A y\|^{2},
$$

for some $\beta>0$;

(iv) $\lambda$-strictly pseudocontractive [6] if, for each $x, y \in C$, there exists $j(x-y) \in J(x-y)$ such that

$$
\langle A x-A y, j(x-y)\rangle \leq\|x-y\|^{2}-\lambda\|x-y-(A x-A y)\|^{2}
$$

for some $\lambda \in(0,1)$. 
It is worth emphasizing that the definition of the inverse strongly accretive mapping is based on that of the inverse strongly monotone mapping, which was studied by so many authors; see, for example, [7-9].

Very recently, Cai and $\mathrm{Bu}[10]$ considered the following general system of variational inequalities (GSVI) in a real smooth Banach space $X$, which involves finding $\left(x^{*}, y^{*}\right) \in$ $C \times C$ such that

$$
\begin{aligned}
& \left\langle\mu_{1} B_{1} y^{*}+x^{*}-y^{*}, J\left(x-x^{*}\right)\right\rangle \geq 0, \quad \forall x \in C, \\
& \left\langle\mu_{2} B_{2} x^{*}+y^{*}-x^{*}, J\left(x-y^{*}\right)\right\rangle \geq 0, \quad \forall x \in C,
\end{aligned}
$$

where $C$ is a nonempty, closed, and convex subset of $X$, $B_{1}, B_{2}: C \rightarrow X$ are two nonlinear mappings, and $\mu_{1}$ and $\mu_{2}$ are two positive constants. Here the set of solutions of GSVI (14) is denoted by $\operatorname{GSVI}\left(C, B_{1}, B_{2}\right)$. In particular, if $X=H$, a real Hilbert space, then GSVI (14) reduces to the following GSVI of finding $\left(x^{*}, y^{*}\right) \in C \times C$ such that

$$
\begin{array}{ll}
\left\langle\mu_{1} B_{1} y^{*}+x^{*}-y^{*}, x-x^{*}\right\rangle \geq 0, & \forall x \in C, \\
\left\langle\mu_{2} B_{2} x^{*}+y^{*}-x^{*}, x-y^{*}\right\rangle \geq 0, & \forall x \in C,
\end{array}
$$

where $\mu_{1}$ and $\mu_{2}$ are two positive constants. The set of solutions of problem (15) is still denoted by $\operatorname{GSVI}\left(C, B_{1}, B_{2}\right)$. In particular, if $B_{1}=B_{2}=A$, then problem (15) reduces to the new system of variational inequalities (NSVI), introduced and studied by Verma [11]. Furthermore, if $x^{*}=y^{*}$ additionally, then the NSVI reduces to the classical variational inequality problem (VIP) of finding $x^{*} \in C$ such that

$$
\left\langle A x^{*}, x-x^{*}\right\rangle \geq 0, \quad \forall x \in C .
$$

The solution set of the VIP $(16)$ is denoted by $\operatorname{VI}(C, A)$. Variational inequality theory has been studied quite extensively and has emerged as an important tool in the study of a wide class of obstacle, unilateral, free, moving, equilibrium problems. It is now well known that the variational inequalities are equivalent to the fixed point problems, the origin of which can be traced back to Lions and Stampacchia [12]. This alternative formulation has been used to suggest and analyze projection iterative method for solving variational inequalities under the conditions that the involved operator must be strongly monotone and Lipschitz continuous.

Recently, Ceng et al. [13] transformed problem (15) into a fixed point problem in the following way.

Lemma 4 (see [13]). For a given $\bar{x}, \bar{y} \in C,(\bar{x}, \bar{y})$ is a solution of problem (15) if and only if $\bar{x}$ is a fixed point of the mapping $G: C \rightarrow C$ defined by

$$
\begin{aligned}
G(x)=P_{C}[ & P_{C}\left(x-\mu_{2} B_{2} x\right) \\
& \left.-\mu_{1} B_{1} P_{C}\left(x-\mu_{2} B_{2} x\right)\right], \quad \forall x \in C,
\end{aligned}
$$

where $\bar{y}=P_{C}\left(\bar{x}-\mu_{2} B_{2} \bar{x}\right)$ and $P_{C}$ is the projection of $H$ onto $C$.

In particular, if the mapping $B_{i}: C \rightarrow H$ is $\beta_{i}$-inverse strongly monotone for $i=1,2$, then the mapping $G$ is nonexpansive provided $\mu_{i} \in\left(0,2 \beta_{i}\right)$ for $i=1,2$.
In 1976, Korpelevič [14] proposed an iterative algorithm for solving the VIP (16) in Euclidean space $\mathbf{R}^{n}$ as follows:

$$
\begin{gathered}
y_{n}=P_{C}\left(x_{n}-\tau A x_{n}\right), \\
x_{n+1}=P_{C}\left(x_{n}-\tau A y_{n}\right), \quad n \geq 0,
\end{gathered}
$$

with $\tau>0$ a given number, which is known as the extragradient method (see also [15]). The literature on the VIP is vast and Korpelevich's extragradient method has received great attention given by many authors, who improved it in various ways; see, for example, $[10,13,16-23]$ the and references therein, to name but a few.

In particular, whenever $X$ is still a real smooth Banach space, $B_{1}=B_{2}=A$, and $x^{*}=y^{*}$, then GSVI (17) reduces to the variational inequality problem (VIP) of finding $x^{*} \in C$ such that

$$
\left\langle A x^{*}, J\left(x-x^{*}\right)\right\rangle \geq 0, \quad \forall x \in C .
$$

which was considered by Aoyama et al. [24]. Note that VIP (19) is connected with the fixed point problem for nonlinear mapping (see, e.g., $[15,25])$, the problem of finding a zero point of a nonlinear operator (see, e.g., $[1,26]$ ), and so on. It is clear that VIP (19) extends VIP (16) from Hilbert spaces to Banach spaces.

In order to find a solution of VIP (19), Aoyama et al. [24] introduced the following iterative scheme for an accretive operator $A$ :

$$
x_{n+1}=\alpha_{n} x_{n}+\left(1-\alpha_{n}\right) \Pi_{C}\left(x_{n}-\lambda_{n} A x_{n}\right), \quad \forall n \geq 1,
$$

where $\Pi_{C}$ is a sunny nonexpansive retraction from $X$ onto $C$. Then they proved a weak convergence theorem.

Beyond doubt, it is an interesting and valuable problem of constructing some algorithms with strong convergence for solving GSVI (14) which contains VIP (19) as a special case. Very recently, Cai and $\mathrm{Bu}[10]$ constructed an iterative algorithm for solving GSVI (14) and a common fixed point problem of an infinite family of nonexpansive mappings in a uniformly convex and 2-uniformly smooth Banach space. They proved the strong convergence of the proposed algorithm by virtue of the following inequality in a 2-uniformly smooth Banach space $X$.

Lemma 5 (see [27]). Let $X$ be a 2-uniformly smooth Banach space. Then

$$
\|x+y\|^{2} \leq\|x\|^{2}+2\langle y, J(x)\rangle+2\|\kappa y\|^{2}, \quad \forall x, y \in X,
$$

where $\kappa$ is the 2-uniformly smooth constant of $X$ and $J$ is the normalized duality mapping from $X$ into $X^{*}$.

Define the mapping $G: C \rightarrow C$ as follows:

$$
G(x):=\Pi_{C}\left(I-\mu_{1} B_{1}\right) \Pi_{C}\left(I-\mu_{2} B_{2}\right) x, \quad \forall x \in C .
$$

The fixed point set of $G$ is denoted by $\Omega$. Then their strong convergence theorem on the proposed method is stated as follows. 
Theorem 6 (see [10, Theorem 3.1]). Let $C$ be a nonempty closed convex subset of a uniformly convex and 2-uniformly smooth Banach space $X$. Let $\Pi_{C}$ be a sunny nonexpansive retraction from $X$ onto $C$. Let the mapping $B_{i}: C \rightarrow X$ be $\beta_{i}$-inverse strongly accretive with $0<\mu_{i}<\beta_{i} / \kappa^{2}$ for $i=1,2$. Let $f$ be a contraction of $C$ into itself with coefficient $\delta \in(0,1)$. Let $\left\{S_{n}\right\}_{n=1}^{\infty}$ be an infinite family of nonexpansive mappings of $C$ into itself such that $F=\bigcap_{i=1}^{\infty} \operatorname{Fix}\left(S_{i}\right) \cap \Omega \neq \emptyset$, where $\Omega$ is the fixed point set of the mapping $G$ defined by (22). For arbitrarily given $x_{1} \in C$, let $\left\{x_{n}\right\}$ be the sequence generated by

$$
\begin{gathered}
x_{n+1}=\beta_{n} x_{n}+\left(1-\beta_{n}\right) S_{n} y_{n}, \\
y_{n}=\alpha_{n} f\left(x_{n}\right)+\left(1-\alpha_{n}\right) z_{n}, \\
z_{n}=\Pi_{C}\left(u_{n}-\mu_{1} B_{1} u_{n}\right), \\
u_{n}=\Pi_{C}\left(x_{n}-\mu_{2} B_{2} x_{n}\right), \quad \forall n \geq 1 .
\end{gathered}
$$

Suppose that $\left\{\alpha_{n}\right\}$ and $\left\{\beta_{n}\right\}$ are two sequences in $(0,1)$ satisfying the following conditions:

$$
\begin{aligned}
& \text { (i) } \lim _{n \rightarrow \infty} \alpha_{n}=0 \text { and } \sum_{n=1}^{\infty} \alpha_{n}=\infty \text {; } \\
& \text { (ii) } 0<\liminf _{n \rightarrow \infty} \beta_{n} \leq \lim \sup _{n \rightarrow \infty} \beta_{n}<1 \text {. }
\end{aligned}
$$

Assume that $\sum_{n=1}^{\infty} \sup _{x \in D}\left\|S_{n+1} x-S_{n} x\right\|<\infty$ for any bounded subset $D$ of $C$ and let $S$ be a mapping of $C$ into $X$ defined by $S x=\lim _{n \rightarrow \infty} S_{n} x$ for all $x \in C$ and suppose that $\operatorname{Fix}(S)=$ $\bigcap_{n=1}^{\infty} \operatorname{Fix}\left(S_{n}\right)$. Then $\left\{x_{n}\right\}$ converges strongly to $q \in F$, which solves the following VIP:

$$
\langle q-f(q), J(q-p)\rangle \leq 0, \quad \forall p \in F .
$$

Corollary 7 (see [10, Corollary 3.2]). Let C be a nonempty closed convex subset of a uniformly convex and 2-uniformly smooth Banach space $X$. Let $\Pi_{C}$ be a sunny nonexpansive retraction from $X$ onto $C$. Let the mapping $B_{i}: C \rightarrow X$ be $\beta_{i}$-inverse strongly accretive with $0<\mu_{i}<\beta_{i} / \kappa^{2}$ for $i=1,2$. Let $f$ be a contraction of $C$ into itself with coefficient $\delta \in(0,1)$. Let $S$ be a nonexpansive mapping of $C$ into itself such that $F=\operatorname{Fix}(S) \cap \Omega \neq \emptyset$, where $\Omega$ is the fixed point set of the mapping $G$ defined by (22). For arbitrarily given $x_{1} \in C$, let $\left\{x_{n}\right\}$ be the sequence generated by

$$
\begin{gathered}
x_{n+1}=\beta_{n} x_{n}+\left(1-\beta_{n}\right) S y_{n}, \\
y_{n}=\alpha_{n} f\left(x_{n}\right)+\left(1-\alpha_{n}\right) z_{n}, \\
z_{n}=\Pi_{C}\left(u_{n}-\mu_{1} B_{1} u_{n}\right), \\
u_{n}=\Pi_{C}\left(x_{n}-\mu_{2} B_{2} x_{n}\right), \quad \forall n \geq 1 .
\end{gathered}
$$

Suppose that $\left\{\alpha_{n}\right\}$ and $\left\{\beta_{n}\right\}$ are two sequences in $(0,1)$ satisfying the following conditions:

(i) $\lim _{n \rightarrow \infty} \alpha_{n}=0$ and $\sum_{n=1}^{\infty} \alpha_{n}=\infty$;

(ii) $0<\liminf _{n \rightarrow \infty} \beta_{n} \leq \lim \sup _{n \rightarrow \infty} \beta_{n}<1$.

Then $\left\{x_{n}\right\}$ converges strongly to $q \in F$, which solves the following VIP:

$$
\langle q-f(q), J(q-p)\rangle \leq 0, \quad \forall p \in F
$$

We remark that in Theorem 6, the Banach space $X$ is both uniformly convex and 2-uniformly smooth. According to Lemma 5, the 2-uniform smoothness of $X$ guarantees the nonexpansivity of the mapping $I-\mu_{i} B_{i}$ for $\alpha_{i}$-inversestrongly accretive mapping $B_{i}: C \rightarrow X$ with $0 \leq \mu_{i} \leq \alpha_{i} / \kappa^{2}$ for $i=1,2$, and hence the composite mapping $G: C \rightarrow C$ is nonexpansive where $G=\Pi_{C}\left(I-\mu_{1} B_{1}\right) \Pi_{C}\left(I-\mu_{2} B_{2}\right)$. In the meantime, for the convenience of implementing the argument techniques in [13], they have applied the following inequality in a real smooth and uniform convex Banach space $X$.

Proposition 8 (see [28]). Let $X$ be a real smooth and uniform convex Banach space and let $r>0$. Then there exists a strictly increasing, continuous, and convex function $g:[0,2 r] \rightarrow \mathbf{R}$, $g(0)=0$ such that

$$
g(\|x-y\|) \leq\|x\|^{2}-2\langle x, J(y)\rangle+\|y\|^{2}, \quad \forall x, y \in B_{r},
$$

where $B_{r}=\{x \in X:\|x\| \leq r\}$.

Let $C$ be a nonempty closed convex subset of a uniformly convex Banach space $X$ which has a uniformly Gateaux differentiable norm. Let $\Pi_{C}$ be a sunny nonexpansive retraction from $X$ onto $C$. Motivated and inspired by the research going on this area, we introduce and analyze hybrid implicit and explicit extragradient methods for finding a zero of an accretive operator $A \subset X \times X$ such that $\overline{D(A)} \subset C \subset$ $\bigcap_{r>0} R(I+r A)$ and solving GSVI (14) and a fixed point problem of an infinite family of nonexpansive self-mappings on $C$. We establish some strong convergence theorems for hybrid implicit and explicit extragradient algorithms under suitable assumptions. Furthermore, we derive the strong convergence of hybrid implicit and explicit extragradient algorithms for finding a common element of the set of zeros of an accretive operator and the common fixed point set of an infinite family of nonexpansive self-mappings on $C$ and a self-mapping whose complement is strictly pseudocontractive and strongly accretive on $C$. The results presented in this paper improve, extend, supplement, and develop the corresponding results announced in the earlier and very recent literature; see, for example, $[5,10,13,16]$.

\section{Preliminaries}

Let $X$ be a real Banach space. $X$ is said to be smooth if the limit

$$
\lim _{t \rightarrow 0} \frac{\|x+t y\|-\|x\|}{t},
$$

exists for all $x, y \in U$; in this case, $X$ is also said to have a Gateaux differentiable norm. $X$ is said to have a uniformly Gateaux differentiable norm if for each $y \in U$, the limit is attained uniformly for $x \in U$. Moreover, it is said to be uniformly smooth if this limit is attained uniformly for $x, y \in$ $U$. The norm of $X$ is said to be the Frechet differential if, for each $x \in U$, this limit is attained uniformly for $y \in U$. In 
addition, we define a function $\rho:[0, \infty) \rightarrow[0, \infty)$ called the modulus of smoothness of $X$ as follows:

$$
\begin{aligned}
\rho(\tau)=\sup \{ & \frac{1}{2}(\|x+y\|+\|x-y\|) \\
& -1: x, y \in X,\|x\|=1,\|y\|=\tau\} .
\end{aligned}
$$

It is known that $X$ is uniformly smooth if and only if $\lim _{\tau \rightarrow 0} \rho(\tau) / \tau=0$. Let $q$ be a fixed real number with $1<q \leq$ 2. Then a Banach space $X$ is said to be $q$-uniformly smooth if there exists a constant $c>0$ such that $\rho(\tau) \leq c \tau^{q}$ for all $\tau>0$. As pointed out in [29], no Banach space is $q$-uniformly smooth for $q>2$.

We list some lemmas that will be used in the sequel. Lemma 9 can be found in [30]. Lemma 10 is an immediate consequence of the subdifferential inequality of the function $(1 / 2)\|\cdot\|^{2}$.

Lemma 9. Let $\left\{s_{n}\right\}$ be a sequence of nonnegative real numbers satisfying

$$
s_{n+1} \leq\left(1-\alpha_{n}\right) s_{n}+\alpha_{n} \beta_{n}+\gamma_{n}, \quad \forall n \geq 0,
$$

where $\left\{\alpha_{n}\right\},\left\{\beta_{n}\right\}$, and $\left\{\gamma_{n}\right\}$ satisfy the following conditions:

(i) $\left\{\alpha_{n}\right\} \subset[0,1]$ and $\sum_{n=0}^{\infty} \alpha_{n}=\infty$;

(ii) $\limsup _{n \rightarrow \infty} \beta_{n} \leq 0$;

(iii) $\gamma_{n} \geq 0$, for all $n \geq 0$, and $\sum_{n=0}^{\infty} \gamma_{n}<\infty$.

Then $\lim \sup _{n \rightarrow \infty} s_{n}=0$.

Lemma 10. In a smooth Banach space $X$, there holds the inequality

$$
\|x+y\|^{2} \leq\|x\|^{2}+2\langle y, J(x+y)\rangle, \quad \forall x, y \in X .
$$

Lemma 11 (see [6]). Let $C$ be a nonempty closed convex subset of a real smooth Banach space $X$ and let $A: C \rightarrow X$ be a $\lambda$ strictly pseudocontractive mapping. Then $A$ is Lipschitz continuous with constant $1+1 / \lambda$.

Proof. Since $A: C \rightarrow X$ is a $\lambda$-strictly pseudocontractive mapping, we have for all $x, y \in C$

$$
\langle A x-A y, J(x-y)\rangle \leq\|x-y\|^{2}-\lambda\|x-y-(A x-A y)\|^{2},
$$

and hence

$$
\begin{aligned}
\lambda\|(I-A) x-(I-A) y\|^{2} \\
\quad \leq\langle(I-A) x-(I-A) y, J(x-y)\rangle .
\end{aligned}
$$

This yields

$$
\|(I-A) x-(I-A) y\| \leq \frac{1}{\lambda}\|x-y\|, \quad \forall x, y \in C .
$$

Therefore,

$$
\begin{aligned}
\|A x-A y\| & \leq\|x-y\|+\|(I-A) x-(I-A) y\| \\
& \leq\left(1+\frac{1}{\lambda}\right)\|x-y\|, \quad \forall x, y \in C .
\end{aligned}
$$

Let $D$ be a subset of $C$ and let $\Pi$ be a mapping of $C$ into $D$. Then $\Pi$ is said to be sunny if

$$
\Pi[\Pi(x)+t(x-\Pi(x))]=\Pi(x),
$$

whenever $\Pi(x)+t(x-\Pi(x)) \in C$ for $x \in C$ and $t \geq 0$. A mapping $\Pi$ of $C$ into itself is called a retraction if $\Pi^{2}=\Pi$. If a mapping $\Pi$ of $C$ into itself is a retraction, then $\Pi(z)=z$ for every $z \in R(\Pi)$ where $R(\Pi)$ is the range of $\Pi$. A subset $D$ of $C$ is called a sunny nonexpansive retract of $C$ if there exists a sunny nonexpansive retraction from $C$ onto $D$. The following lemma concerns the sunny nonexpansive retraction.

Lemma 12 (see [31]). Let $C$ be a nonempty closed convex subset of a real smooth Banach space X. Let D be a nonempty subset of $C$. Let $\Pi$ be a retraction of $C$ onto $D$. Then the following are equivalent:

(i) $\Pi$ is sunny and nonexpansive;

(ii) $\|\Pi(x)-\Pi(y)\|^{2} \leq\langle x-y, J(\Pi(x)-\Pi(y))\rangle$, for all $x, y \in C$;

(iii) $\langle x-\Pi(x), J(y-\Pi(x))\rangle \leq 0$, for all $x \in C, y \in D$.

It is well known that if $X=H$ a Hilbert space, then a sunny nonexpansive retraction $\Pi_{C}$ is coincident with the metric projection from $X$ onto $C$; that is, $\Pi_{C}=P_{C}$. If $C$ is a nonempty closed convex subset of a strictly convex and uniformly smooth Banach space $X$ and if $T: C \rightarrow C$ is a nonexpansive mapping with the fixed point set $\operatorname{Fix}(T) \neq \emptyset$, then the set $\operatorname{Fix}(T)$ is a sunny nonexpansive retract of $C$.

Lemma 13 (see [32]). Let $X$ be a uniformly convex Banach space and $B_{r}=\{x \in X:\|x\| \leq r\}, r>0$. Then there exists a continuous, strictly increasing, and convex function $g:[0$, $\infty] \rightarrow[0, \infty], g(0)=0$ such that

$$
\begin{aligned}
\|\alpha x+\beta y+\gamma z\|^{2} \leq & \alpha\|x\|^{2}+\beta\|y\|^{2} \\
& +\gamma\|z\|^{2}-\alpha \beta g(\|x-y\|)
\end{aligned}
$$

for all $x, y, z \in B_{r}$ and all $\alpha, \beta, \gamma \in[0,1]$ with $\alpha+\beta+\gamma=1$.

Lemma 14 (see [33]). Let $C$ be a nonempty closed convex subset of a Banach space $X$. Let $S_{0}, S_{1}, \ldots$ be a sequence of mappings of $C$ into itself. Suppose that $\sum_{n=1}^{\infty} \sup \left\{\| S_{n} x-\right.$ $\left.S_{n-1} x \|: x \in C\right\}<\infty$. Then for each $y \in C,\left\{S_{n} y\right\}$ converges strongly to some point of $C$. Moreover, let $S$ be a mapping of $C$ into itself defined by $S y=\lim _{n \rightarrow \infty} S_{n} y$ for all $y \in C$. Then $\lim _{n \rightarrow \infty} \sup \left\{\left\|S x-S_{n} x\right\|: x \in C\right\}=0$.

Let $C$ be a nonempty closed convex subset of a Banach space $X$ and let $T: C \rightarrow C$ be a nonexpansive mapping with $\operatorname{Fix}(T) \neq \emptyset$. As previously mentioned, let $\Xi_{C}$ be the set of all contractions on $C$. For $t \in(0,1)$ and $f \in \Xi_{C}$, let $x_{t} \in C$ be the unique fixed point of the contraction $x \mapsto t f(x)+(1-t) T x$ on $C$; that is,

$$
x_{t}=t f\left(x_{t}\right)+(1-t) T x_{t}
$$


Lemma 15 (see [15]). Let $X$ be a reflexive and strictly convex Banach space with a uniformly Gateaux differentiable norm. Let $C$ a nonempty closed convex subset of $X, T: C \rightarrow C$ a nonexpansive mapping with $\operatorname{Fix}(T) \neq \emptyset$, and $f \in \Xi_{C}$. Then the net $\left\{x_{t}\right\}$ defined by $x_{t}=t f\left(x_{t}\right)+(1-t) T x_{t}$ converges strongly to a point in $\operatorname{Fix}(T)$. If we define a mapping $Q: \Xi_{C} \rightarrow \operatorname{Fix}(T)$ by $Q(f):=s-\lim _{t \rightarrow 0} x_{t}$, for all $f \in \Xi_{C}$, then $Q(f)$ solves the following VIP:

$$
\begin{array}{r}
\langle(I-f) Q(f), J(Q(f)-p)\rangle \leq 0, \\
\forall f \in \Xi_{C}, p \in \operatorname{Fix}(T) .
\end{array}
$$

Lemma 16 (see [34]). Let $C$ be a nonempty closed convex subset of a strictly convex Banach space $X$. Let $\left\{T_{n}\right\}_{n=0}^{\infty}$ be a sequence of nonexpansive mappings on $C$. Suppose $\bigcap_{n=0}^{\infty} \operatorname{Fix}\left(T_{n}\right)$ is nonempty. Let $\left\{\lambda_{n}\right\}$ be a sequence of positive numbers with $\sum_{n=0}^{\infty} \lambda_{n}=1$. Then a mapping $S$ on $C$ defined by $S x=\sum_{n=0}^{\infty} \lambda_{n} T_{n} x$ for $x \in C$ is well defined, nonexpansive and $\operatorname{Fix}(S)=\bigcap_{n=0}^{\infty} \operatorname{Fix}\left(T_{n}\right)$ holds.

Lemma 17 (see [27]). Given a number $r>0$. A real Banach space $X$ is uniformly convex if and only if there exists a continuous strictly increasing function $g:[0, \infty) \rightarrow[0, \infty)$, $g(0)=0$, such that

$$
\begin{aligned}
\|\lambda x+(1-\lambda) y\|^{2} \leq & \lambda\|x\|^{2}+(1-\lambda)\|y\|^{2} \\
& -\lambda(1-\lambda) g(\|x-y\|)
\end{aligned}
$$

for all $\lambda \in[0,1]$ and $x, y \in X$ such that $\|x\| \leq r$ and $\|y\| \leq r$.

Lemma 18 (see [24]). Let $C$ be a nonempty closed convex subset of a smooth Banach space $X$. Let $\Pi_{C}$ be a sunny nonexpansive retraction from $X$ onto $C$ and let $A$ be an accretive operator of $C$ into $X$. Then, for all $\lambda>0$,

$$
\operatorname{VI}(C, A)=\operatorname{Fix}\left(\Pi_{C}(I-\lambda A)\right) .
$$

Lemma 19. Let $C$ be a nonempty closed convex subset of a smooth Banach space $X$ and let the mapping $B_{i}: C \rightarrow X$ be $\zeta_{i}$ strictly pseudocontractive and $\theta_{i}$ strongly accretive with $\theta_{i}+\zeta_{i} \geq 1$ for $i=1,2$. Then, for $\mu_{i} \in(0,1]$, we have

$$
\begin{aligned}
&\left\|\left(I-\mu_{i} B_{i}\right) x-\left(I-\mu_{i} B_{i}\right) y\right\| \\
& \leq\left\{\sqrt{\frac{1-\theta_{i}}{\zeta_{i}}}+\left(1-\mu_{i}\right)\left(1+\frac{1}{\zeta_{i}}\right)\right\} \\
& \times\|x-y\|, \quad \forall x, y \in C,
\end{aligned}
$$

for $i=1$, 2. In particular, if $1-\left(\zeta_{i} /\left(1+\zeta_{i}\right)\right)\left(1-\sqrt{\left(1-\theta_{i}\right) / \zeta_{i}}\right) \leq$ $\mu_{i} \leq 1$, then $I-\mu_{i} B_{i}$ is nonexpansive for $i=1,2$.

Proof. Taking into account the $\zeta_{i}$-strict pseudocontractivity of $B_{i}$, by Lemma 11 we derive for every $x, y \in C$

$$
\left\|B_{i} x-B_{i} y\right\| \leq\left(1+\frac{1}{\zeta_{i}}\right)\|x-y\| .
$$

Utilizing the $\theta_{i}$-strong accretivity and $\zeta_{i}$-strict pseudocontractivity of $B_{i}$, we get

$$
\begin{aligned}
\zeta_{i} \|(I & \left.-B_{i}\right) x-\left(I-B_{i}\right) y \|^{2} \\
& \leq\|x-y\|^{2}-\left\langle B_{i} x-B_{i} y, J(x-y)\right\rangle \\
& \leq\left(1-\theta_{i}\right)\|x-y\|^{2} .
\end{aligned}
$$

So, we have

$$
\left\|\left(I-B_{i}\right) x-\left(I-B_{i}\right) y\right\| \leq \sqrt{\frac{1-\theta_{i}}{\zeta_{i}}}\|x-y\| .
$$

Therefore, for $\mu_{i} \in(0,1]$, we have

$$
\begin{aligned}
\|(I- & \left.\mu_{i} B_{i}\right) x-\left(I-\mu_{i} B_{i}\right) y \| \\
\leq & \left\|\left(I-B_{i}\right) x-\left(I-B_{i}\right) y\right\| \\
& +\left(1-\mu_{i}\right)\left\|B_{i} x-B_{i} y\right\| \\
\leq & \sqrt{\frac{1-\theta_{i}}{\zeta_{i}}}\|x-y\|+\left(1-\mu_{i}\right)\left(1+\frac{1}{\zeta_{i}}\right)\|x-y\| \\
= & \left\{\sqrt{\frac{1-\theta_{i}}{\zeta_{i}}}+\left(1-\mu_{i}\right)\left(1+\frac{1}{\zeta_{i}}\right)\right\}\|x-y\| .
\end{aligned}
$$

Since $1-\left(\zeta_{i} /\left(1+\zeta_{i}\right)\right)\left(1-\sqrt{\left(1-\theta_{i}\right) / \zeta_{i}}\right) \leq \mu_{i} \leq 1$, it follows immediately that

$$
\sqrt{\frac{1-\theta_{i}}{\zeta_{i}}}+\left(1-\mu_{i}\right)\left(1+\frac{1}{\zeta_{i}}\right) \leq 1
$$

This implies that $I-\mu_{i} B_{i}$ is nonexpansive for $i=1,2$.

Lemma 20. Let $C$ be a nonempty closed convex subset of a smooth Banach space $X$. Let $\Pi_{C}$ be a sunny nonexpansive retraction from $X$ onto $C$ and let the mapping $B_{i}: C \rightarrow X$ be $\zeta_{i}$ strictly pseudocontractive and $\theta_{i}$ strongly accretive with $\theta_{i}+\zeta_{i} \geq 1$ for $i=1,2$. Let $G: C \rightarrow C$ be the mapping defined by

$$
\begin{aligned}
G(x)=\Pi_{C}[ & \Pi_{C}\left(x-\mu_{2} B_{2} x\right) \\
& \left.-\mu_{1} B_{1} \Pi_{C}\left(x-\mu_{2} B_{2} x\right)\right], \quad \forall x \in C .
\end{aligned}
$$

If $1-\left(\zeta_{i} /\left(1+\zeta_{i}\right)\right)\left(1-\sqrt{\left(1-\theta_{i}\right) / \zeta_{i}}\right) \leq \mu_{i} \leq 1$, then $G: C \rightarrow C$ is nonexpansive. 
Proof. According to Lemma 19, we know that $I-\mu_{i} B_{i}$ is nonexpansive for $i=1,2$. Hence, for all $x, y \in C$, we have

$$
\begin{gathered}
\|G(x)-G(y)\|=\| \Pi_{C}\left[\Pi_{C}\left(x-\mu_{2} B_{2} x\right)\right. \\
\left.-\mu_{1} B_{1} \Pi_{C}\left(x-\mu_{2} B_{2} x\right)\right] \\
-\Pi_{C}\left[\Pi_{C}\left(y-\mu_{2} B_{2} y\right)\right. \\
\left.\quad-\mu_{1} B_{1} \Pi_{C}\left(y-\mu_{2} B_{2} y\right)\right] \| \\
=\| \Pi_{C}\left(I-\mu_{1} B_{1}\right) \Pi_{C}\left(I-\mu_{2} B_{2}\right) x \\
\quad-\Pi_{C}\left(I-\mu_{1} B_{1}\right) \Pi_{C}\left(I-\mu_{2} B_{2}\right) y \| \\
\leq \|\left(I-\mu_{1} B_{1}\right) \Pi_{C}\left(I-\mu_{2} B_{2}\right) x \\
\quad-\left(I-\mu_{1} B_{1}\right) \Pi_{C}\left(I-\mu_{2} B_{2}\right) y \| \\
\leq\left\|\Pi_{C}\left(I-\mu_{2} B_{2}\right) x-\Pi_{C}\left(I-\mu_{2} B_{2}\right) y\right\| \\
\leq\left\|\left(I-\mu_{2} B_{2}\right) x-\left(I-\mu_{2} B_{2}\right) y\right\| \\
\leq\|x-y\| .
\end{gathered}
$$

This shows that $G: C \rightarrow C$ is nonexpansive. This completes the proof.

Lemma 21. Let $C$ be a nonempty closed convex subset of a smooth Banach space $X$. Let $\Pi_{C}$ be a sunny nonexpansive retraction from $X$ onto $C$ and let $B_{1}, B_{2}: C \rightarrow X$ be two nonlinear mappings. For a given $x^{*}, y^{*} \in C,\left(x^{*}, y^{*}\right)$ is a solution of GSVI (14) if and only if $x^{*}=\Pi_{C}\left(y^{*}-\mu_{1} B_{1} y^{*}\right)$ where $y^{*}=\Pi_{C}\left(x^{*}-\mu_{2} B_{2} x^{*}\right)$.

Proof. We can rewrite GSVI (14) as

$$
\begin{aligned}
& \left\langle x^{*}-\left(y^{*}-\mu_{1} B_{1} y^{*}\right), J\left(x-x^{*}\right)\right\rangle \geq 0, \quad \forall x \in C, \\
& \left\langle y^{*}-\left(x^{*}-\mu_{2} B_{2} x^{*}\right), J\left(x-y^{*}\right)\right\rangle \geq 0, \quad \forall x \in C,
\end{aligned}
$$

which is obviously equivalent to

$$
\begin{aligned}
& x^{*}=\Pi_{C}\left(y^{*}-\mu_{1} B_{1} y^{*}\right), \\
& y^{*}=\Pi_{C}\left(x^{*}-\mu_{2} B_{2} x^{*}\right),
\end{aligned}
$$

because of Lemma 12. This completes the proof.

Remark 22. By Lemma 21, we observe that

$$
x^{*}=\Pi_{C}\left[\Pi_{C}\left(x^{*}-\mu_{2} B_{2} x^{*}\right)-\mu_{1} B_{1} \Pi_{C}\left(x^{*}-\mu_{2} B_{2} x^{*}\right)\right],
$$

which implies that $x^{*}$ is a fixed point of the mapping $G=$ $\Pi_{C}\left(I-\mu_{1} B_{1}\right) \Pi_{C}\left(I-\mu_{2} B_{2}\right)$. Throughout this paper, the set of fixed points of the mapping $G$ is denoted by $\Omega$.

\section{Hybrid Implicit Extragradient Algorithm}

In this section, let $C$ be a nonempty closed convex subset of a uniformly convex Banach space $X$ which has a uniformly
Gateaux differentiable norm. We suggest and analyze a hybrid implicit extragradient algorithm for finding a zero of $A \subset X \times$ $X$ an accretive operator in $X$ with $\overline{D(A)} \subset C \subset \bigcap_{r>0} R(I+$ $r A)$ and solving a general system of variational inequalities and a common fixed point problem of an infinite family of nonexpansive self-mappings in $X$.

Theorem 23. Let $C$ be a nonempty closed convex subset of a uniformly convex Banach space $X$ which has a uniformly Gateaux differentiable norm. Let $\Pi_{C}$ be a sunny nonexpansive retraction from $X$ onto $C$. Let $A \subset X \times X$ be an accretive operator in $X$ such that $\overline{D(A)} \subset C \subset \bigcap_{r>0} R(I+r A)$. Let $B_{i}: C \rightarrow X$ be $\zeta_{i}$ strictly pseudocontractive and $\theta_{i}$ strongly accretive with $\theta_{i}+\zeta_{i} \geq 1$ for each $i=1,2$. Define the mapping $G: C \rightarrow C$ by $G=\Pi_{C}\left(I-\mu_{1} B_{1}\right) \Pi_{C}\left(I-\mu_{2} B_{2}\right)$ where $1-\left(\zeta_{i} /\left(1+\zeta_{i}\right)\right)\left(1-\sqrt{\left(1-\theta_{i}\right) / \zeta_{i}}\right) \leq \mu_{i} \leq 1$ for $i=1,2$. Let $f: C \rightarrow C$ be a contraction with coefficient $\rho \in(0,1)$. Let $\left\{S_{i}\right\}_{i=0}^{\infty}$ be an infinite family of nonexpansive mappings of $C$ into itself such that $F=\bigcap_{i=0}^{\infty} \operatorname{Fix}\left(S_{i}\right) \cap \Omega \cap A^{-1} 0 \neq \emptyset$ where $\Omega=\operatorname{Fix}(G)$. For arbitrarily given $x_{0} \in C$, let $\left\{x_{n}\right\}$ be the sequence generated by

$$
\begin{array}{r}
y_{n}=\alpha_{n} f\left(x_{n}\right)+\beta_{n} x_{n}+\gamma_{n} J_{r_{n}} x_{n}+\delta_{n} S_{n} y_{n}, \\
x_{n+1}=\sigma_{n} y_{n}+\left(1-\sigma_{n}\right) \Pi_{C}\left(I-\mu_{1} B_{1}\right) \\
\times \Pi_{C}\left(I-\mu_{2} B_{2}\right) y_{n}, \quad \forall n \geq 0 .
\end{array}
$$

Suppose that $\left\{r_{n}\right\} \subset(0, \infty),\left\{\sigma_{n}\right\},\left\{\alpha_{n}\right\},\left\{\beta_{n}\right\},\left\{\gamma_{n}\right\},\left\{\delta_{n}\right\} \subset[0,1]$, $\alpha_{n}+\beta_{n}+\gamma_{n}+\delta_{n}=1$ and the following conditions hold:

(i) $\lim _{n \rightarrow \infty} \alpha_{n}=0$ and $\sum_{n=0}^{\infty} \alpha_{n}=\infty$;

(ii) $\left\{\gamma_{n}\right\},\left\{\delta_{n}\right\} \subset[c, d]$ for some $c, d \in(0,1)$;

(iii) $\sum_{n=1}^{\infty}\left(\left|\sigma_{n}-\sigma_{n-1}\right|+\left|\alpha_{n}-\alpha_{n-1}\right|+\left|\beta_{n}-\beta_{n-1}\right|+\left|\gamma_{n}-\gamma_{n-1}\right|+\right.$ $\left.\left|\delta_{n}-\delta_{n-1}\right|\right)<\infty$

(iv) $\sum_{n=1}^{\infty}\left|r_{n}-r_{n-1}\right|<\infty$ and $r_{n} \geq \varepsilon>0$, for all $n \geq 0$ for some $\varepsilon>0$

(v) $0<\liminf _{n \rightarrow \infty} \beta_{n} \leq \limsup _{n \rightarrow \infty} \beta_{n}<1$ and $0<$ $\liminf _{n \rightarrow \infty} \sigma_{n} \leq \limsup _{n \rightarrow \infty} \sigma_{n}<1$.

Assume that $\sum_{n=0}^{\infty} \sup _{x \in D}\left\|S_{n+1} x-S_{n} x\right\|<\infty$ for any bounded subset $D$ of $C$ and let $S$ be a mapping of $C$ into itself defined by $S x=\lim _{n \rightarrow \infty} S_{n} x$ for all $x \in C$ and suppose that $\operatorname{Fix}(S)=$ $\bigcap_{i=0}^{\infty} \operatorname{Fix}\left(S_{i}\right)$. Then $\left\{x_{n}\right\}$ converges strongly to $q \in F$, which solves the following VIP:

$$
\langle q-f(q), J(q-p)\rangle \leq 0, \quad \forall p \in F .
$$

Proof. It is easy to see that (53) can be rewritten as follows:

$$
\begin{gathered}
y_{n}=\alpha_{n} f\left(x_{n}\right)+\beta_{n} J_{r_{n}} x_{n}+\gamma_{n} B x_{n}+\delta_{n} S_{n} y_{n}, \\
x_{n+1}=\sigma_{n} y_{n}+\left(1-\sigma_{n}\right) G y_{n}, \quad \forall n \geq 0,
\end{gathered}
$$

where $G=\Pi_{C}\left(I-\mu_{1} B_{1}\right) \Pi_{C}\left(I-\mu_{2} B_{2}\right)$. By Lemma 20 we know that $G$ is a nonexpansive self-mapping on $C$. 
Now, let us show that the sequence $\left\{x_{n}\right\}$ is bounded. Indeed, take a fixed $p \in F$ arbitrarily. Then from (55), we have

$$
\begin{aligned}
\left\|y_{n}-p\right\| \leq & \alpha_{n}\left\|f\left(x_{n}\right)-p\right\|+\beta_{n}\left\|x_{n}-p\right\| \\
& +\gamma_{n}\left\|J_{r_{n}} x_{n}-p\right\|+\delta_{n}\left\|S_{n} y_{n}-p\right\| \\
\leq & \alpha_{n}\left(\left\|f\left(x_{n}\right)-f(p)\right\|+\|f(p)-p\|\right) \\
& +\beta_{n}\left\|x_{n}-p\right\|+\gamma_{n}\left\|x_{n}-p\right\| \\
& +\delta_{n}\left\|y_{n}-p\right\| \\
\leq & \alpha_{n}\left(\rho\left\|x_{n}-p\right\|+\|f(p)-p\|\right) \\
& +\beta_{n}\left\|x_{n}-p\right\|+\gamma_{n}\left\|x_{n}-p\right\| \\
& +\delta_{n}\left\|y_{n}-p\right\| \\
= & \left(1-\delta_{n}-(1-\rho) \alpha_{n}\right)\left\|x_{n}-p\right\| \\
& +\alpha_{n}\|f(p)-p\|+\delta_{n}\left\|y_{n}-p\right\|,
\end{aligned}
$$

which hence implies that

$$
\begin{aligned}
\left\|y_{n}-p\right\| \leq & \left(1-\frac{(1-\rho) \alpha_{n}}{1-\delta_{n}}\right)\left\|x_{n}-p\right\| \\
& +\frac{\alpha_{n}}{1-\delta_{n}}\|f(p)-p\| .
\end{aligned}
$$

So, we have

$$
\begin{aligned}
\left\|x_{n+1}-p\right\| \leq & \sigma_{n}\left\|y_{n}-p\right\| \\
& +\left(1-\sigma_{n}\right)\left\|G y_{n}-p\right\| \\
\leq & \sigma_{n}\left\|y_{n}-p\right\|+\left(1-\sigma_{n}\right)\left\|y_{n}-p\right\| \\
= & \left\|y_{n}-p\right\| \\
\leq & \left(1-\frac{(1-\rho) \alpha_{n}}{1-\delta_{n}}\right)\left\|x_{n}-p\right\| \\
& +\frac{\alpha_{n}}{1-\delta_{n}}\|f(p)-p\| \\
= & \left(1-\frac{(1-\rho) \alpha_{n}}{1-\delta_{n}}\right)\left\|x_{n}-p\right\| \\
& +\frac{(1-\rho) \alpha_{n}\|f(p)-p\|}{1-\delta_{n}} \frac{\| f}{1-\rho} \\
\leq & \max \left\{\left\|x_{n}-p\right\|, \frac{\|f(p)-p\|}{1-\rho}\right\} .
\end{aligned}
$$

By induction, we obtain

$$
\left\|x_{n}-p\right\| \leq \max \left\{\left\|x_{0}-p\right\|, \frac{\|f(p)-p\|}{1-\rho}\right\}, \quad \forall n \geq 0 .
$$

Hence $\left\{x_{n}\right\}$ is bounded and so are $\left\{y_{n}\right\},\left\{G y_{n}\right\},\left\{f\left(x_{n}\right)\right\},\left\{J_{r_{n}} x_{n}\right\}$, and $\left\{S_{n} y_{n}\right\}$.
Let us show that

$$
\lim _{n \rightarrow \infty}\left\|x_{n+1}-x_{n}\right\|=0
$$

As a matter of fact, observe that $y_{n}$ can be rewritten as follows:

$$
y_{n}=\beta_{n} x_{n}+\left(1-\beta_{n}\right) z_{n},
$$

where $z_{n}=\left(\alpha_{n} f\left(x_{n}\right)+\gamma_{n} J_{r_{n}} x_{n}+\delta_{n} S_{n} y_{n}\right) /\left(1-\beta_{n}\right)$. Observe that

$$
\begin{aligned}
&\left\|z_{n}-z_{n-1}\right\|= \| \frac{\alpha_{n} f\left(x_{n}\right)+\gamma_{n} J_{r_{n}} x_{n}+\delta_{n} S_{n} y_{n}}{1-\beta_{n}} \\
&-\frac{\alpha_{n-1} f\left(x_{n-1}\right)+\gamma_{n-1} J_{r_{n-1}} x_{n-1}+\delta_{n-1} S_{n-1} y_{n-1}}{1-\beta_{n-1}} \| \\
&=\left\|\frac{y_{n}-\beta_{n} x_{n}}{1-\beta_{n}}-\frac{y_{n-1}-\beta_{n-1} x_{n-1}}{1-\beta_{n-1}}\right\| \\
&= \| \frac{y_{n}-\beta_{n} x_{n}}{1-\beta_{n}}-\frac{y_{n-1}-\beta_{n-1} x_{n-1}}{1-\beta_{n}} \\
&+\frac{y_{n-1}-\beta_{n-1} x_{n-1}}{1-\beta_{n}}-\frac{y_{n-1}-\beta_{n-1} x_{n-1}}{1-\beta_{n-1}} \| \\
& \leq\left\|\frac{y_{n}-\beta_{n} x_{n}}{1-\beta_{n}}-\frac{y_{n-1}-\beta_{n-1} x_{n-1}}{1-\beta_{n}}\right\| \\
&+\left\|\frac{y_{n-1}-\beta_{n-1} x_{n-1}}{1-\beta_{n}}-\frac{y_{n-1}-\beta_{n-1} x_{n-1}}{1-\beta_{n-1}}\right\| \\
&= \frac{1}{1-\beta_{n}}\left\|y_{n}-\beta_{n} x_{n}-\left(y_{n-1}-\beta_{n-1} x_{n-1}\right)\right\| \\
&+\left|\frac{1}{1-\beta_{n}}-\frac{1}{1-\beta_{n-1}}\right|\left\|y_{n-1}-\beta_{n-1} x_{n-1}\right\| f\left(x_{n}\right)-f\left(x_{n-1}\right) \| \\
&= \frac{1}{1-\beta_{n}}\left\|y_{n}-\beta_{n} x_{n}-\left(y_{n-1}-\beta_{n-1} x_{n-1}\right)\right\| \\
&+\frac{\mid \beta_{n} x_{n}-J_{r_{n-1}} x_{n-1} \|}{\left(1-\beta_{n-1}\right)\left(1-\beta_{n}\right)}\left\|\alpha_{n}-S_{n-1} y_{n-1}\right\| \\
&+\alpha_{n-1} f\left(x_{n}\right)+\beta_{n-1} x_{n-1} \| \\
& J_{r_{n}} x_{n}+\delta_{n} S_{n} y_{n}
\end{aligned}
$$




$$
\begin{aligned}
& +\left|\alpha_{n}-\alpha_{n-1}\right|\left\|f\left(x_{n-1}\right)\right\| \\
& +\left|\gamma_{n}-\gamma_{n-1}\right|\left\|J_{r_{n-1}} x_{n-1}\right\| \\
& \left.+\left|\delta_{n}-\delta_{n-1}\right|\left\|S_{n-1} y_{n-1}\right\|\right] \\
& +\frac{\left|\beta_{n}-\beta_{n-1}\right|}{\left(1-\beta_{n-1}\right)\left(1-\beta_{n}\right)} \\
& \times\left\|y_{n-1}-\beta_{n-1} x_{n-1}\right\| .
\end{aligned}
$$

On the other hand, if $r_{n-1} \leq r_{n}$, using the resolvent identity in Proposition 1,

$$
J_{r_{n}} x_{n}=J_{r_{n-1}}\left(\frac{r_{n-1}}{r_{n}} x_{n}+\left(1-\frac{r_{n-1}}{r_{n}}\right) J_{r_{n}} x_{n}\right),
$$

we get

$$
\begin{aligned}
\left\|J_{r_{n}} x_{n}-J_{r_{n-1}} x_{n-1}\right\| & \\
= & \left\|J_{r_{n-1}}\left(\frac{r_{n-1}}{r_{n}} x_{n}+\left(1-\frac{r_{n-1}}{r_{n}}\right) J_{r_{n}} x_{n}\right)-J_{r_{n-1}} x_{n-1}\right\| \\
& \leq \frac{r_{n-1}}{r_{n}}\left\|x_{n}-x_{n-1}\right\|+\left(1-\frac{r_{n-1}}{r_{n}}\right)\left\|J_{r_{n}} x_{n}-x_{n-1}\right\| \\
& \leq\left\|x_{n}-x_{n-1}\right\|+\frac{r_{n}-r_{n-1}}{r_{n}}\left\|J_{r_{n}} x_{n}-x_{n-1}\right\| \\
& \leq\left\|x_{n}-x_{n-1}\right\|+\frac{1}{\varepsilon}\left|r_{n}-r_{n-1}\right|\left\|J_{r_{n}} x_{n}-x_{n-1}\right\| .
\end{aligned}
$$

If $r_{n} \leq r_{n-1}$, we derive in a similar way

$$
\begin{aligned}
\left\|J_{r_{n}} x_{n}-J_{r_{n-1}} x_{n-1}\right\| \leq & \left\|x_{n-1}-x_{n}\right\| \\
& +\frac{1}{\varepsilon}\left|r_{n-1}-r_{n}\right|\left\|J_{r_{n-1}} x_{n-1}-x_{n}\right\| .
\end{aligned}
$$

Thus, combining the above cases we obtain

$$
\begin{aligned}
\left\|J_{r_{n}} x_{n}-J_{r_{n-1}} x_{n-1}\right\| \leq & \left\|x_{n-1}-x_{n}\right\| \\
& +M_{0}\left|r_{n-1}-r_{n}\right|, \quad \forall n \geq 1,
\end{aligned}
$$

where $\sup _{n \geq 1}\left\{(1 / \varepsilon)\left(\left\|J_{r_{n}} x_{n}-x_{n-1}\right\|+\left\|J_{r_{n-1}} x_{n-1}-x_{n}\right\|\right)\right\} \leq M_{0}$ for some $M_{0}>0$. Substituting (66) into (62), we have

$$
\begin{aligned}
& \left\|z_{n}-z_{n-1}\right\| \leq \frac{1}{1-\beta_{n}}\left[\alpha_{n}\left\|f\left(x_{n}\right)-f\left(x_{n-1}\right)\right\|\right. \\
& +\gamma_{n}\left(\left\|x_{n-1}-x_{n}\right\|+M_{0}\left|r_{n-1}-r_{n}\right|\right) \\
& +\delta_{n}\left\|S_{n} y_{n}-S_{n-1} y_{n-1}\right\| \\
& +\left|\alpha_{n}-\alpha_{n-1}\right|\left\|f\left(x_{n-1}\right)\right\| \\
& +\left|\gamma_{n}-\gamma_{n-1}\right| \mid\left\|J_{r_{n-1}} x_{n-1}\right\| \\
& \left.+\left|\delta_{n}-\delta_{n-1}\right|\left\|S_{n-1} y_{n-1}\right\|\right] \\
& +\frac{\left|\beta_{n}-\beta_{n-1}\right|}{\left(1-\beta_{n-1}\right)\left(1-\beta_{n}\right)}\left\|y_{n-1}-\beta_{n-1} x_{n-1}\right\| \\
& \leq \frac{1}{1-\beta_{n}}\left[\alpha_{n} \rho\left\|x_{n}-x_{n-1}\right\|\right. \\
& +\gamma_{n}\left(\left\|x_{n-1}-x_{n}\right\|+M_{0}\left|r_{n-1}-r_{n}\right|\right) \\
& +\delta_{n}\left\|S_{n} y_{n}-S_{n} y_{n-1}\right\| \\
& +\left|\alpha_{n}-\alpha_{n-1}\right|\left\|f\left(x_{n-1}\right)\right\| \\
& +\left|\gamma_{n}-\gamma_{n-1}\right||| J_{r_{n-1}} x_{n-1} \| \\
& +\left|\delta_{n}-\delta_{n-1}\right|\left\|S_{n-1} y_{n-1}\right\| \\
& \left.+\delta_{n}\left\|S_{n} y_{n-1}-S_{n-1} y_{n-1}\right\|\right] \\
& +\frac{\left|\beta_{n}-\beta_{n-1}\right|}{\left(1-\beta_{n-1}\right)\left(1-\beta_{n}\right)} \\
& \times \| \alpha_{n-1} f\left(x_{n-1}\right)+\gamma_{n-1} J_{r_{n-1}} x_{n-1} \\
& +\delta_{n-1} S_{n-1} x_{n-1} \| \\
& \leq \frac{1}{1-\beta_{n}}\left[\left(\alpha_{n} \rho+\gamma_{n}\right)\left\|x_{n}-x_{n-1}\right\|\right. \\
& +\delta_{n}\left\|y_{n}-y_{n-1}\right\| \\
& +M\left(\left|\alpha_{n}-\alpha_{n-1}\right|+\left|\gamma_{n}-\gamma_{n-1}\right|\right. \\
& \left.+\left|\delta_{n}-\delta_{n-1}\right|+\left|r_{n-1}-r_{n}\right|\right) \\
& \left.+\delta_{n}\left\|S_{n} y_{n-1}-S_{n-1} y_{n-1}\right\|\right] \\
& +\frac{1}{\left(1-\beta_{n-1}\right)\left(1-\beta_{n}\right)}\left|\beta_{n}-\beta_{n-1}\right| M
\end{aligned}
$$

where $\sup _{n \geq 0}\left\{M_{0}+\left\|f\left(x_{n}\right)\right\|+\left\|J_{r_{n}} x_{n}\right\|+\left\|S_{n} y_{n}\right\|\right\} \leq M$ for some $M>0$. In the meantime, simple calculations show that

$$
\begin{aligned}
y_{n}-y_{n-1}= & \beta_{n}\left(x_{n}-x_{n-1}\right)+\left(1-\beta_{n}\right)\left(z_{n}-z_{n-1}\right) \\
& +\left(\beta_{n}-\beta_{n-1}\right)\left(x_{n-1}-z_{n-1}\right) .
\end{aligned}
$$


Taking into account condition (iv), we may assume, without loss of generality, that $\left\{\beta_{n}\right\} \subset[a, b]$ for some $a, b \in(0,1)$. Hence, from (67) and (68) we deduce that

$$
\begin{aligned}
& \left\|y_{n}-y_{n-1}\right\| \leq \beta_{n}\left\|x_{n}-x_{n-1}\right\| \\
& +\left(1-\beta_{n}\right)\left\|z_{n}-z_{n-1}\right\| \\
& +\left|\beta_{n}-\beta_{n-1}\right|\left\|x_{n-1}-z_{n-1}\right\| \\
& \leq \beta_{n}\left\|x_{n}-x_{n-1}\right\|+\left(1-\beta_{n}\right) \\
& \times\left\{\frac { 1 } { 1 - \beta _ { n } } \left[\left(\alpha_{n} \rho+\gamma_{n}\right)\left\|x_{n}-x_{n-1}\right\|\right.\right. \\
& +\delta_{n}\left\|y_{n}-y_{n-1}\right\| \\
& +M\left(\left|\alpha_{n}-\alpha_{n-1}\right|+\left|\gamma_{n}-\gamma_{n-1}\right|\right. \\
& \left.+\left|\delta_{n}-\delta_{n-1}\right|+\left|r_{n-1}-r_{n}\right|\right) \\
& \left.+\delta_{n}\left\|S_{n} y_{n-1}-S_{n-1} y_{n-1}\right\|\right] \\
& \left.+\frac{1}{\left(1-\beta_{n-1}\right)\left(1-\beta_{n}\right)}\left|\beta_{n}-\beta_{n-1}\right| M\right\} \\
& +\left|\beta_{n}-\beta_{n-1}\right|\left\|x_{n-1}-z_{n-1}\right\| \\
& =\left(\alpha_{n} \rho+\beta_{n}+\gamma_{n}\right)\left\|x_{n}-x_{n-1}\right\| \\
& +\delta_{n}\left\|y_{n}-y_{n-1}\right\| \\
& +M\left(\left|\alpha_{n}-\alpha_{n-1}\right|+\left|\gamma_{n}-\gamma_{n-1}\right|\right. \\
& \left.+\left|\delta_{n}-\delta_{n-1}\right|+\left|r_{n-1}-r_{n}\right|\right) \\
& +\delta_{n}\left\|S_{n} y_{n-1}-S_{n-1} y_{n-1}\right\| \\
& +\frac{1}{1-\beta_{n-1}}\left|\beta_{n}-\beta_{n-1}\right| M \\
& +\left|\beta_{n}-\beta_{n-1}\right|\left\|x_{n-1}-z_{n-1}\right\| \\
& =\left(1-\delta_{n}-\alpha_{n}(1-\rho)\right)\left\|x_{n}-x_{n-1}\right\| \\
& +\delta_{n}\left\|y_{n}-y_{n-1}\right\| \\
& +M\left(\left|\alpha_{n}-\alpha_{n-1}\right|+\left|\gamma_{n}-\gamma_{n-1}\right|\right. \\
& \left.+\left|\delta_{n}-\delta_{n-1}\right|+\left|r_{n-1}-r_{n}\right|\right) \\
& +\delta_{n}\left\|S_{n} y_{n-1}-S_{n-1} y_{n-1}\right\| \\
& +\frac{1}{1-\beta_{n-1}}\left|\beta_{n}-\beta_{n-1}\right| M \\
& +\left|\beta_{n}-\beta_{n-1}\right|\left\|x_{n-1}-z_{n-1}\right\| \\
& \leq\left(1-\delta_{n}-\alpha_{n}(1-\rho)\right)\left\|x_{n}-x_{n-1}\right\| \\
& +\delta_{n}\left\|y_{n}-y_{n-1}\right\| \\
& +\widetilde{M}_{0}\left(\left|\alpha_{n}-\alpha_{n-1}\right|\right. \\
& +\left|\beta_{n}-\beta_{n-1}\right|+\left|\gamma_{n}-\gamma_{n-1}\right|
\end{aligned}
$$

$$
\begin{aligned}
& \left.+\left|\delta_{n}-\delta_{n-1}\right|+\left|r_{n-1}-r_{n}\right|\right) \\
& +\delta_{n}\left\|S_{n} y_{n-1}-S_{n-1} y_{n-1}\right\|,
\end{aligned}
$$

where $\sup _{n \geq 0}\left\{(M /(1-b))+\left\|x_{n}-z_{n}\right\|\right\} \leq \widetilde{M}_{0}$ for some $\widetilde{M}_{0}>0$. Thus, from condition (ii) we immediately get

$$
\begin{aligned}
\left\|y_{n}-y_{n-1}\right\| \leq & \left(1-\frac{1-\rho}{1-\delta_{n}} \alpha_{n}\right)\left\|x_{n}-x_{n-1}\right\| \\
& +\frac{\widetilde{M}_{0}}{1-\delta_{n}}\left(\left|\alpha_{n}-\alpha_{n-1}\right|\right. \\
& +\left|\beta_{n}-\beta_{n-1}\right|+\left|\gamma_{n}-\gamma_{n-1}\right| \\
& \left.+\left|\delta_{n}-\delta_{n-1}\right|+\left|r_{n-1}-r_{n}\right|\right) \\
& +\frac{\delta_{n}}{1-\delta_{n}}\left\|S_{n} y_{n-1}-S_{n-1} y_{n-1}\right\| \\
\leq & \left(1-\frac{1-\rho}{1-\delta_{n}} \alpha_{n}\right)\left\|x_{n}-x_{n-1}\right\| \\
& +\frac{\widetilde{M}_{0}}{1-d}\left(\left|\alpha_{n}-\alpha_{n-1}\right|\right. \\
& +\frac{d}{1-d} \| S_{n} y_{n-1}-\beta_{n-1}|+| \gamma_{n}-\gamma_{n-1} \mid \\
& \left.+\left|\delta_{n}-\delta_{n-1}\right|+\left|r_{n-1}-r_{n}\right|\right)
\end{aligned}
$$

Also, from (55) we have

$$
\begin{aligned}
x_{n+1}-x_{n}= & \sigma_{n}\left(y_{n}-y_{n-1}\right) \\
& +\left(\sigma_{n}-\sigma_{n-1}\right)\left(y_{n-1}-G y_{n-1}\right) \\
& +\left(1-\sigma_{n}\right)\left(G y_{n}-G y_{n-1}\right) .
\end{aligned}
$$

Taking into account condition (v), we may assume, without loss of generality, that $\left\{\beta_{n}\right\} \subset[a, b]$ for some $a, b \in(0,1)$. This together with (70) implies that

$$
\begin{aligned}
\left\|x_{n+1}-x_{n}\right\| \leq & \sigma_{n}\left\|y_{n}-y_{n-1}\right\| \\
& +\left|\sigma_{n}-\sigma_{n-1}\right|\left\|y_{n-1}-G y_{n-1}\right\| \\
& +\left(1-\sigma_{n}\right)\left\|G y_{n}-G y_{n-1}\right\| \\
\leq & \sigma_{n}\left\|y_{n}-y_{n-1}\right\| \\
& +\left|\sigma_{n}-\sigma_{n-1}\right|\left\|y_{n-1}-G y_{n-1}\right\| \\
& +\left(1-\sigma_{n}\right)\left\|y_{n}-y_{n-1}\right\| \\
= & \left\|y_{n}-y_{n-1}\right\| \\
& +\left|\sigma_{n}-\sigma_{n-1}\right|\left\|y_{n-1}-G y_{n-1}\right\|
\end{aligned}
$$




$$
\begin{aligned}
& \leq\left(1-\frac{1-\rho}{1-\delta_{n}} \alpha_{n}\right)\left\|x_{n}-x_{n-1}\right\| \\
& +\frac{\widetilde{M}_{0}}{1-d}\left(\left|\alpha_{n}-\alpha_{n-1}\right|\right. \\
& +\left|\beta_{n}-\beta_{n-1}\right|+\left|\gamma_{n}-\gamma_{n-1}\right| \\
& \left.+\left|\delta_{n}-\delta_{n-1}\right|+\left|r_{n-1}-r_{n}\right|\right) \\
& +\frac{d}{1-d}\left\|S_{n} y_{n-1}-S_{n-1} y_{n-1}\right\| \\
& +\left|\sigma_{n}-\sigma_{n-1}\right|\left\|y_{n-1}-G y_{n-1}\right\| \\
& \leq\left(1-\frac{1-\rho}{1-\delta_{n}} \alpha_{n}\right)\left\|x_{n}-x_{n-1}\right\| \\
& +M_{1}\left(\left|\sigma_{n}-\sigma_{n-1}\right|+\left|\alpha_{n}-\alpha_{n-1}\right|\right. \\
& +\left|\beta_{n}-\beta_{n-1}\right|+\left|\gamma_{n}-\gamma_{n-1}\right| \\
& \left.+\left|\delta_{n}-\delta_{n-1}\right|+\left|r_{n-1}-r_{n}\right|\right) \\
& +\frac{d}{1-d}\left\|S_{n} y_{n-1}-S_{n-1} y_{n-1}\right\| \text {, }
\end{aligned}
$$

where $\sup _{n \geq 0}\left\{\left(\widetilde{M}_{0} /(1-d)\right)+\left\|y_{n}-G y_{n}\right\|\right\} \leq M_{1}$ for some $M_{1}>$ 0 . Since $\sum_{n=0}^{\infty} \alpha_{n}=\infty$ and $\left((1-\rho) /\left(1-\delta_{n}\right)\right) \alpha_{n} \geq(1-\rho) \alpha_{n}$, we know that $\sum_{n=0}^{\infty}\left((1-\rho) /\left(1-\delta_{n}\right)\right) \alpha_{n}=\infty$. So, applying Lemma 9 to (72), we obtain from conditions (iii), (iv), and the assumption on $\left\{S_{n}\right\}$ that

$$
\lim _{n \rightarrow \infty}\left\|x_{n+1}-x_{n}\right\|=0
$$

Next we show that $\left\|x_{n}-G x_{n}\right\| \rightarrow 0$ as $n \rightarrow \infty$. Indeed, according to Lemma 10 we have from (55)

$$
\begin{aligned}
\left\|y_{n}-p\right\|^{2}=\| & \alpha_{n}\left(f\left(x_{n}\right)-f(p)\right) \\
& +\beta_{n}\left(x_{n}-p\right)+\gamma_{n}\left(J_{r_{n}} x_{n}-p\right) \\
& +\delta_{n}\left(S_{n} y_{n}-p\right)+\alpha_{n}(f(p)-p) \|^{2} \\
\leq & \| \alpha_{n}\left(f\left(x_{n}\right)-f(p)\right)+\beta_{n}\left(x_{n}-p\right) \\
& +\gamma_{n}\left(J_{r_{n}} x_{n}-p\right)+\delta_{n}\left(S_{n} y_{n}-p\right) \|^{2} \\
& +2 \alpha_{n}\left\langle f(p)-p, J\left(y_{n}-p\right)\right\rangle \\
\leq & \alpha_{n}\left\|f\left(x_{n}\right)-f(p)\right\|^{2} \\
& +\beta_{n}\left\|x_{n}-p\right\|^{2}+\gamma_{n}\left\|J_{r_{n}} x_{n}-p\right\|^{2} \\
& +\delta_{n}\left\|S_{n} y_{n}-p\right\|^{2} \\
& +2 \alpha_{n}\left\langle f(p)-p, J\left(y_{n}-p\right)\right\rangle
\end{aligned}
$$

$$
\begin{aligned}
\leq & \alpha_{n} \rho\left\|x_{n}-p\right\|^{2}+\beta_{n}\left\|x_{n}-p\right\|^{2} \\
& +\gamma_{n}\left\|x_{n}-p\right\|^{2}+\delta_{n}\left\|y_{n}-p\right\|^{2} \\
& +2 \alpha_{n}\left\langle f(p)-p, J\left(y_{n}-p\right)\right\rangle \\
= & \left(\alpha_{n} \rho+\beta_{n}+\gamma_{n}\right)\left\|x_{n}-p\right\|^{2} \\
& +\delta_{n}\left\|y_{n}-p\right\|^{2}+2 \alpha_{n}\left\langle f(p)-p, J\left(y_{n}-p\right)\right\rangle \\
\leq & \left(1-\delta_{n}-\alpha_{n}(1-\rho)\right)\left\|x_{n}-p\right\|^{2} \\
& +\delta_{n}\left\|y_{n}-p\right\|^{2}+2 \alpha_{n}\left\langle f(p)-p, J\left(y_{n}-p\right)\right\rangle,
\end{aligned}
$$

which hence implies that

$$
\begin{aligned}
\left\|y_{n}-p\right\|^{2} \leq & \left(1-\frac{\alpha_{n}(1-\rho)}{1-\delta_{n}}\right)\left\|x_{n}-p\right\|^{2} \\
& +\frac{\alpha_{n}}{1-\delta_{n}} 2\left\langle f(p)-p, J\left(y_{n}-p\right)\right\rangle \\
\leq & \left(1-\frac{\alpha_{n}(1-\rho)}{1-\delta_{n}}\right)\left\|x_{n}-p\right\|^{2} \\
& +\frac{\alpha_{n}}{1-\delta_{n}} 2\|f(p)-p\|\left\|y_{n}-p\right\| .
\end{aligned}
$$

Utilizing Lemma 17, we get from (55) and (75)

$$
\begin{aligned}
\left\|x_{n+1}-p\right\|^{2}= & \| \sigma_{n}\left(y_{n}-p\right) \\
& +\left(1-\sigma_{n}\right)\left(G y_{n}-p\right) \|^{2} \\
\leq & \sigma_{n}\left\|y_{n}-p\right\|^{2}+\left(1-\sigma_{n}\right)\left\|G y_{n}-p\right\|^{2} \\
& -\sigma_{n}\left(1-\sigma_{n}\right) g_{1}\left(\left\|y_{n}-G y_{n}\right\|\right) \\
\leq & \sigma_{n}\left\|y_{n}-p\right\|^{2}+\left(1-\sigma_{n}\right)\left\|y_{n}-p\right\|^{2} \\
& -\sigma_{n}\left(1-\sigma_{n}\right) g_{1}\left(\left\|y_{n}-G y_{n}\right\|\right) \\
= & \left\|y_{n}-p\right\|^{2}-\sigma_{n}\left(1-\sigma_{n}\right) g_{1}\left(\left\|y_{n}-G y_{n}\right\|\right) \\
\leq & \left(1-\frac{\alpha_{n}(1-\rho)}{1-\delta_{n}}\right)\left\|x_{n}-p\right\|^{2} \\
& +\frac{\alpha_{n}}{1-\delta_{n}} 2\|f(p)-p\|\left\|y_{n}-p\right\| \\
& -\sigma_{n}\left(1-\sigma_{n}\right) g_{1}\left(\left\|y_{n}-G y_{n}\right\|\right) \\
\leq & \left\|x_{n}-p\right\|^{2} \\
& +\frac{\alpha_{n}}{1-\delta_{n}} 2\|f(p)-p\|\left\|y_{n}-p\right\| \\
& -\sigma_{n}\left(1-\sigma_{n}\right) g_{1}\left(\left\|y_{n}-G y_{n}\right\|\right) \\
&
\end{aligned}
$$


which hence yields

$$
\begin{aligned}
\sigma_{n}(1- & \left.\sigma_{n}\right) g_{1}\left(\left\|y_{n}-G y_{n}\right\|\right) \\
\leq & \left\|x_{n}-p\right\|^{2}-\left\|x_{n+1}-p\right\|^{2} \\
& +\frac{\alpha_{n}}{1-\delta_{n}} 2\|f(p)-p\|\left\|y_{n}-p\right\| \\
\leq & \left(\left\|x_{n}-p\right\|+\left\|x_{n+1}-p\right\|\right) \\
& \times\left\|x_{n}-x_{n+1}\right\| \\
& +\frac{\alpha_{n}}{1-d} 2\|f(p)-p\|\left\|y_{n}-p\right\| .
\end{aligned}
$$

Since $\alpha_{n} \rightarrow 0$ and $\left\|x_{n+1}-x_{n}\right\| \rightarrow 0$, from condition (iv) and the boundedness of $\left\{x_{n}\right\}$ and $\left\{y_{n}\right\}$, it follows that

$$
\lim _{n \rightarrow \infty} g_{1}\left(\left\|y_{n}-G y_{n}\right\|\right)=0
$$

Utilizing the properties of $g_{1}$, we have

$$
\lim _{n \rightarrow \infty}\left\|y_{n}-G y_{n}\right\|=0
$$

Observe that

$$
\begin{aligned}
\left\|x_{n}-y_{n}\right\| \leq & \left\|x_{n}-x_{n+1}\right\| \\
& +\left\|x_{n+1}-y_{n}\right\|=\left\|x_{n}-x_{n+1}\right\| \\
& +\left(1-\sigma_{n}\right)\left\|G y_{n}-y_{n}\right\| \longrightarrow 0, \quad \text { as } n \longrightarrow \infty,
\end{aligned}
$$

and hence

$$
\begin{aligned}
\left\|x_{n}-G x_{n}\right\| \leq & \left\|x_{n}-y_{n}\right\|+\left\|y_{n}-G y_{n}\right\| \\
& +\left\|G y_{n}-G x_{n}\right\| \leq 2\left\|x_{n}-y_{n}\right\| \\
& +\left\|y_{n}-G y_{n}\right\| \longrightarrow 0, \quad \text { as } n \longrightarrow \infty .
\end{aligned}
$$

That is,

$$
\lim _{n \rightarrow \infty}\left\|x_{n}-y_{n}\right\|=0, \quad \lim _{n \rightarrow \infty}\left\|x_{n}-G x_{n}\right\|=0 .
$$

Next, let us show that

$$
\lim _{n \rightarrow \infty}\left\|J_{r_{n}} x_{n}-x_{n}\right\|=0, \quad \quad \lim _{n \rightarrow \infty}\left\|S_{n} x_{n}-x_{n}\right\|=0 .
$$

Indeed, observe that $y_{n}$ can be rewritten as follows:

$$
\begin{aligned}
y_{n}= & \alpha_{n} f\left(x_{n}\right)+\beta_{n} x_{n}+\gamma_{n} J_{r_{n}} x_{n}+\delta_{n} S_{n} y_{n} \\
= & \alpha_{n} f\left(x_{n}\right)+\beta_{n} x_{n} \\
& +\left(\gamma_{n}+\delta_{n}\right) \frac{\gamma_{n} J_{r_{n}} x_{n}+\delta_{n} S_{n} y_{n}}{\gamma_{n}+\delta_{n}} \\
= & \alpha_{n} f\left(x_{n}\right)+\beta_{n} x_{n}+e_{n} \widehat{z}_{n},
\end{aligned}
$$

where $e_{n}=\gamma_{n}+\delta_{n}$ and $\widehat{z}_{n}=\left(\gamma_{n} J_{r_{n}} x_{n}+\delta_{n} S_{n} y_{n}\right) /\left(\gamma_{n}+\delta_{n}\right)$. Utilizing Lemma 13 and (84), we have

$$
\begin{aligned}
& \left\|y_{n}-p\right\|^{2}=\| \alpha_{n}\left(f\left(x_{n}\right)-p\right) \\
& +\beta_{n}\left(x_{n}-p\right)+e_{n}\left(\widehat{z}_{n}-p\right) \|^{2} \\
& \leq \alpha_{n}\left\|f\left(x_{n}\right)-p\right\|^{2} \\
& +\beta_{n}\left\|x_{n}-p\right\|^{2}+e_{n}\left\|\widehat{z}_{n}-p\right\|^{2} \\
& -\beta_{n} e_{n} g_{3}\left(\left\|\widehat{z}_{n}-x_{n}\right\|\right) \\
& =\alpha_{n}\left\|f\left(x_{n}\right)-p\right\|^{2} \\
& +\beta_{n}\left\|x_{n}-p\right\|^{2}-\beta_{n} e_{n} g_{2}\left(\left\|\widehat{z}_{n}-x_{n}\right\|\right) \\
& +e_{n}\left\|\frac{\gamma_{n} J_{r_{n}} x_{n}+\delta_{n} S_{n} y_{n}}{\gamma_{n}+\delta_{n}}-p\right\|^{2} \\
& =\alpha_{n}\left\|f\left(x_{n}\right)-p\right\|^{2}+\beta_{n}\left\|x_{n}-p\right\|^{2} \\
& -\beta_{n} e_{n} g_{2}\left(\left\|\widehat{z}_{n}-x_{n}\right\|\right) \\
& +e_{n} \| \frac{\gamma_{n}}{\gamma_{n}+\delta_{n}}\left(J_{r_{n}} x_{n}-p\right) \\
& +\frac{\delta_{n}}{\gamma_{n}+\delta_{n}}\left(S_{n} y_{n}-p\right) \|^{2} \\
& \leq \alpha_{n}\left\|f\left(x_{n}\right)-p\right\|^{2}+\beta_{n}\left\|x_{n}-p\right\|^{2} \\
& -\beta_{n} e_{n} g_{2}\left(\left\|\widehat{z}_{n}-x_{n}\right\|\right) \\
& +e_{n}\left[\frac{\gamma_{n}}{\gamma_{n}+\delta_{n}}\left\|J_{r_{n}} x_{n}-p\right\|^{2}\right. \\
& \left.+\frac{\delta_{n}}{\gamma_{n}+\delta_{n}}\left\|S_{n} y_{n}-p\right\|^{2}\right] \\
& \leq \alpha_{n}\left\|f\left(x_{n}\right)-p\right\|^{2}+\beta_{n}\left\|x_{n}-p\right\|^{2} \\
& -\beta_{n} e_{n} g_{2}\left(\left\|\widehat{z}_{n}-x_{n}\right\|\right) \\
& +e_{n}\left[\frac{\gamma_{n}}{\gamma_{n}+\delta_{n}}\left\|x_{n}-p\right\|\right. \\
& \left.+\frac{\delta_{n}}{\gamma_{n}+\delta_{n}}\left\|y_{n}-p\right\|^{2}\right] \\
& =\alpha_{n}\left\|f\left(x_{n}\right)-p\right\|^{2}+\left(\beta_{n}+\gamma_{n}\right)\left\|x_{n}-p\right\|^{2} \\
& +\delta_{n}\left\|y_{n}-p\right\|^{2}-\beta_{n} e_{n} g_{2}\left(\left\|\widehat{z}_{n}-x_{n}\right\|\right) \\
& =\alpha_{n}\left\|f\left(x_{n}\right)-p\right\|^{2} \\
& +\left(1-\alpha_{n}-\delta_{n}\right)\left\|x_{n}-p\right\|^{2} \\
& +\delta_{n}\left\|y_{n}-p\right\|^{2}-\beta_{n} e_{n} g_{2}\left(\left\|\widehat{z}_{n}-x_{n}\right\|\right)
\end{aligned}
$$




$$
\begin{aligned}
\leq & \alpha_{n}\left\|f\left(x_{n}\right)-p\right\|^{2}+\left(1-\delta_{n}\right)\left\|x_{n}-p\right\|^{2} \\
& +\delta_{n}\left\|y_{n}-p\right\|^{2}-\beta_{n} e_{n} g_{2}\left(\left\|\widehat{z}_{n}-x_{n}\right\|\right),
\end{aligned}
$$

which hence implies that

$$
\begin{aligned}
\beta_{n} e_{n} g_{2}\left(\left\|\bar{z}_{n}-x_{n}\right\|\right) \leq & \alpha_{n}\left\|f\left(x_{n}\right)-p\right\|^{2} \\
& +\left(1-\delta_{n}\right)\left(\left\|x_{n}-p\right\|^{2}-\left\|y_{n}-p\right\|^{2}\right) \\
\leq & \alpha_{n}\left\|f\left(x_{n}\right)-p\right\|^{2} \\
& +\left(1-\delta_{n}\right)\left(\left\|x_{n}-p\right\|\right. \\
& \left.+\left\|y_{n}-p\right\|\right)\left\|x_{n}-y_{n}\right\| \\
\leq & \alpha_{n}\left\|f\left(x_{n}\right)-p\right\|^{2} \\
& +\left(\left\|x_{n}-p\right\|+\left\|y_{n}-p\right\|\right)\left\|x_{n}-y_{n}\right\| .
\end{aligned}
$$

Utilizing (82), conditions (i), (ii), and (v), and the boundedness of $\left\{x_{n}\right\},\left\{y_{n}\right\}$, and $\left\{f\left(x_{n}\right)\right\}$, we get

$$
\lim _{n \rightarrow \infty} g_{2}\left(\left\|\bar{z}_{n}-x_{n}\right\|\right)=0 .
$$

From the properties of $g_{2}$, we have

$$
\lim _{n \rightarrow \infty}\left\|\widehat{z}_{n}-x_{n}\right\|=0
$$

Utilizing Lemma 17 and the definition of $\widehat{z}_{n}$, we have

$$
\begin{aligned}
\left\|\bar{z}_{n}-p\right\|^{2}= & \left\|\frac{\gamma_{n} J_{r_{n}} x_{n}+\delta_{n} S_{n} y_{n}}{\gamma_{n}+\delta_{n}}-p\right\|^{2} \\
= & \| \frac{\gamma_{n}}{\gamma_{n}+\delta_{n}}\left(J_{r_{n}} x_{n}-p\right) \\
& +\frac{\delta_{n}}{\gamma_{n}+\delta_{n}}\left(S_{n} y_{n}-p\right) \|^{2} \\
\leq & \frac{\gamma_{n}}{\gamma_{n}+\delta_{n}}\left\|J_{r_{n}} x_{n}-p\right\|^{2} \\
& +\frac{\delta_{n}}{\gamma_{n}+\delta_{n}}\left\|S_{n} y_{n}-p\right\|^{2} \\
& -\frac{\gamma_{n} \delta_{n}}{\left(\gamma_{n}+\delta_{n}\right)^{2}} g_{3}\left(\left\|J_{r_{n}} x_{n}-S_{n} y_{n}\right\|\right) \\
\leq & \frac{\gamma_{n}}{\gamma_{n}+\delta_{n}}\left\|x_{n}-p\right\|^{2} \\
& +\frac{\delta_{n}}{\gamma_{n}+\delta_{n}}\left\|y_{n}-p\right\|^{2} \\
& -\frac{\gamma_{n} \delta_{n}}{\left(\gamma_{n}+\delta_{n}\right)^{2}} g_{3}\left(\left\|J_{r_{n}} x_{n}-S_{n} y_{n}\right\|\right),
\end{aligned}
$$

which leads to

$$
\begin{aligned}
& \frac{\gamma_{n} \delta_{n}}{\left(\gamma_{n}+\delta_{n}\right)^{2}} g_{3}\left(\left\|J_{r_{n}} x_{n}-S_{n} x_{n}\right\|\right) \\
& \leq \frac{\gamma_{n}}{\gamma_{n}+\delta_{n}}\left(\left\|x_{n}-p\right\|^{2}-\left\|\widehat{z}_{n}-p\right\|^{2}\right) \\
& \quad+\frac{\delta_{n}}{\gamma_{n}+\delta_{n}}\left(\left\|y_{n}-p\right\|^{2}-\left\|\bar{z}_{n}-p\right\|^{2}\right) \\
& \leq \frac{\gamma_{n}}{\gamma_{n}+\delta_{n}}\left(\left\|x_{n}-p\right\|+\left\|\widehat{z}_{n}-p\right\|\right)\left\|x_{n}-\widehat{z}_{n}\right\| \\
& \quad+\frac{\delta_{n}}{\gamma_{n}+\delta_{n}}\left(\left\|y_{n}-p\right\|+\left\|\widehat{z}_{n}-p\right\|\right)\left\|y_{n}-\widehat{z}_{n}\right\| \\
& \leq \frac{\gamma_{n}}{\gamma_{n}+\delta_{n}}\left(\left\|x_{n}-p\right\|+\left\|\widehat{z}_{n}-p\right\|\right)\left\|x_{n}-\widehat{z}_{n}\right\| \\
&+\frac{\delta_{n}}{\gamma_{n}+\delta_{n}}\left(\left\|y_{n}-p\right\|+\left\|\bar{z}_{n}-p\right\|\right) \\
& \times\left(\left\|y_{n}-x_{n}\right\|+\left\|x_{n}-\widehat{z}_{n}\right\|\right) .
\end{aligned}
$$

Since $\left\{x_{n}\right\},\left\{y_{n}\right\}$, and $\left\{\widehat{z}_{n}\right\}$ are bounded, $\left\|x_{n}-y_{n}\right\| \rightarrow 0$, and $\left\|\widehat{z}_{n}-x_{n}\right\| \rightarrow 0$ as $n \rightarrow \infty$, we deduce from condition (ii) that

$$
\lim _{n \rightarrow \infty} g_{3}\left(\left\|J_{r_{n}} x_{n}-S_{n} y_{n}\right\|\right)=0
$$

From the properties of $g_{3}$, we have

$$
\lim _{n \rightarrow \infty}\left\|J_{r_{n}} x_{n}-S_{n} y_{n}\right\|=0
$$

On the other hand, $y_{n}$ can also be rewritten as follows:

$$
\begin{aligned}
y_{n}= & \alpha_{n} f\left(x_{n}\right)+\beta_{n} x_{n}+\gamma_{n} J_{r_{n}} x_{n}+\delta_{n} S_{n} y_{n} \\
= & \beta_{n} x_{n}+\gamma_{n} J_{r_{n}} x_{n} \\
& +\left(\alpha_{n}+\delta_{n}\right) \frac{\alpha_{n} f\left(x_{n}\right)+\delta_{n} S_{n} y_{n}}{\alpha_{n}+\delta_{n}} \\
= & \beta_{n} x_{n}+\gamma_{n} J_{r_{n}} x_{n}+d_{n} \widetilde{z}_{n},
\end{aligned}
$$


where $d_{n}=\alpha_{n}+\delta_{n}$ and $\widetilde{z}_{n}=\left(\alpha_{n} f\left(x_{n}\right)+\delta_{n} S_{n} y_{n}\right) /\left(\alpha_{n}+\delta_{n}\right)$. Utilizing Lemma 13 and the convexity of $\|\cdot\|^{2}$, we have

$$
\begin{aligned}
& \left\|y_{n}-p\right\|^{2}=\| \beta_{n}\left(x_{n}-p\right) \\
& +\gamma_{n}\left(J_{r_{n}} x_{n}-p\right)+d_{n}\left(\widetilde{z}_{n}-p\right) \|^{2} \\
& \leq \beta_{n}\left\|x_{n}-p\right\|^{2}+\gamma_{n}\left\|J_{r_{n}} x_{n}-p\right\|^{2} \\
& +d_{n}\left\|\tilde{z}_{n}-p\right\|^{2}-\beta_{n} \gamma_{n} g_{4}\left(\left\|x_{n}-J_{r_{n}} x_{n}\right\|\right) \\
& =\beta_{n}\left\|x_{n}-p\right\|^{2}+\gamma_{n}\left\|J_{r_{n}} x_{n}-p\right\|^{2} \\
& +d_{n}\left\|\frac{\alpha_{n} f\left(x_{n}\right)+\delta_{n} S_{n} y_{n}}{\alpha_{n}+\delta_{n}}-p\right\|^{2} \\
& -\beta_{n} \gamma_{n} g_{4}\left(\left\|x_{n}-J_{r_{n}} x_{n}\right\|\right) \\
& =\beta_{n}\left\|x_{n}-p\right\|^{2}+\gamma_{n}\left\|J_{r_{n}} x_{n}-p\right\|^{2} \\
& +d_{n} \| \frac{\alpha_{n}}{\alpha_{n}+\delta_{n}}\left(f\left(x_{n}\right)-p\right) \\
& +\frac{\delta_{n}}{\alpha_{n}+\delta_{n}}\left(S_{n} y_{n}-p\right) \|^{2} \\
& -\beta_{n} \gamma_{n} g_{4}\left(\left\|x_{n}-J_{r_{n}} x_{n}\right\|\right) \\
& \leq \beta_{n}\left\|x_{n}-p\right\|^{2}+\gamma_{n}\left\|x_{n}-p\right\|^{2} \\
& +d_{n}\left[\frac{\alpha_{n}}{\alpha_{n}+\delta_{n}}\left\|f\left(x_{n}\right)-p\right\|^{2}\right. \\
& \left.+\frac{\delta_{n}}{\alpha_{n}+\delta_{n}}\left\|S_{n} y_{n}-p\right\|^{2}\right] \\
& -\beta_{n} \gamma_{n} g_{4}\left(\left\|x_{n}-J_{r_{n}} x_{n}\right\|\right) \\
& \leq \alpha_{n}\left\|f\left(x_{n}\right)-p\right\|^{2} \\
& +\left(\beta_{n}+\gamma_{n}\right)\left\|x_{n}-p\right\|^{2}+\delta_{n}\left\|y_{n}-p\right\|^{2} \\
& -\beta_{n} \gamma_{n} g_{4}\left(\left\|x_{n}-J_{r_{n}} x_{n}\right\|\right) \\
& =\alpha_{n}\left\|f\left(x_{n}\right)-p\right\|^{2} \\
& +\left(1-\alpha_{n}-\delta_{n}\right)\left\|x_{n}-p\right\|^{2} \\
& +\delta_{n}\left\|y_{n}-p\right\|^{2}-\beta_{n} \gamma_{n} g_{4}\left(\left\|x_{n}-J_{r_{n}} x_{n}\right\|\right) \\
& \leq \alpha_{n}\left\|f\left(x_{n}\right)-p\right\|^{2} \\
& +\left(1-\delta_{n}\right)\left\|x_{n}-p\right\|^{2}+\delta_{n}\left\|y_{n}-p\right\|^{2} \\
& -\beta_{n} \gamma_{n} g_{4}\left(\left\|x_{n}-J_{r_{n}} x_{n}\right\|\right) \text {, }
\end{aligned}
$$

which hence implies that

$$
\begin{aligned}
\beta_{n} \gamma_{n} g_{4}\left(\left\|x_{n}-J_{r_{n}} x_{n}\right\|\right) \leq & \alpha_{n}\left\|f\left(x_{n}\right)-p\right\|^{2} \\
& +\left(1-\delta_{n}\right)\left(\left\|x_{n}-p\right\|^{2}-\left\|y_{n}-p\right\|^{2}\right) \\
\leq & \alpha_{n}\left\|f\left(x_{n}\right)-p\right\|^{2} \\
& +\left(1-\delta_{n}\right)\left(\left\|x_{n}-p\right\|+\left\|y_{n}-p\right\|\right) \\
& \times\left\|x_{n}-y_{n}\right\| \leq \alpha_{n}\left\|f\left(x_{n}\right)-p\right\|^{2} \\
& +\left(\left\|x_{n}-p\right\|+\left\|y_{n}-p\right\|\right)\left\|x_{n}-y_{n}\right\| .
\end{aligned}
$$

From (82), conditions (i), (ii), and (v), and the boundedness of $\left\{x_{n}\right\},\left\{y_{n}\right\}$, and $\left\{f\left(x_{n}\right)\right\}$, we have

$$
\lim _{n \rightarrow \infty} g_{4}\left(\left\|x_{n}-J_{r_{n}} x_{n}\right\|\right)=0 .
$$

Utilizing the properties of $g_{4}$, we have

$$
\lim _{n \rightarrow \infty}\left\|x_{n}-J_{r_{n}} x_{n}\right\|=0 .
$$

Note that

$$
\begin{aligned}
\left\|x_{n}-S_{n} x_{n}\right\| \leq & \left\|x_{n}-J_{r_{n}} x_{n}\right\| \\
& +\left\|J_{r_{n}} x_{n}-S_{n} y_{n}\right\| \\
& +\left\|S_{n} y_{n}-S_{n} x_{n}\right\| \\
\leq & \left\|x_{n}-J_{r_{n}} x_{n}\right\| \\
& +\left\|J_{r_{n}} x_{n}-S_{n} y_{n}\right\| \\
& +\left\|y_{n}-x_{n}\right\| .
\end{aligned}
$$

From (82), (92), and (97), we get

$$
\lim _{n \rightarrow \infty}\left\|x_{n}-S_{n} x_{n}\right\|=0 .
$$

In terms of (99) and Lemma 14, we have

$$
\begin{aligned}
\left\|x_{n}-S x_{n}\right\| \leq & \left\|x_{n}-S_{n} x_{n}\right\| \\
& +\left\|S_{n} x_{n}-S x_{n}\right\| \longrightarrow 0, \quad \text { as } n \longrightarrow \infty .
\end{aligned}
$$

That is,

$$
\lim _{n \rightarrow \infty}\left\|x_{n}-S x_{n}\right\|=0 .
$$

Furthermore, we claim that $\lim _{n \rightarrow \infty}\left\|x_{n}-J_{r} x_{n}\right\|=0$ for a fixed number $r$ such that $\varepsilon>r>0$. In fact, from the resolvent identity in Proposition 1, we have

$$
\begin{aligned}
\left\|J_{r_{n}} x_{n}-J_{r} x_{n}\right\| \\
\quad=\left\|J_{r}\left(\frac{r}{r_{n}} x_{n}+\left(1-\frac{r}{r_{n}}\right) J_{r_{n}} x_{n}\right)-J_{r} x_{n}\right\| \\
\quad \leq\left(1-\frac{r}{r_{n}}\right)\left\|x_{n}-J_{r_{n}} x_{n}\right\| \\
\quad \leq\left\|x_{n}-J_{r_{n}} x_{n}\right\| .
\end{aligned}
$$


So, we get from (102)

$$
\begin{aligned}
\left\|x_{n}-J_{r} x_{n}\right\| & \leq\left\|x_{n}-J_{r_{n}} x_{n}\right\|+\left\|J_{r_{n}} x_{n}-J_{r} x_{n}\right\| \\
& \leq\left\|x_{n}-J_{r_{n}} x_{n}\right\|+\left\|x_{n}-J_{r_{n}} x_{n}\right\| \\
& =2\left\|x_{n}-J_{r_{n}} x_{n}\right\| .
\end{aligned}
$$

Thus, from (97) it follows that

$$
\lim _{n \rightarrow \infty}\left\|x_{n}-J_{r} x_{n}\right\|=0
$$

Define a mapping $W x=\left(1-\theta_{1}-\theta_{2}\right) J_{r} x+\theta_{1} S x+\theta_{2} G x$, where $\theta_{1}, \theta_{2} \in(0,1)$ are two constants with $\theta_{1}+\theta_{2}<1$. Then, by Lemma 16, we have that $\operatorname{Fix}(W)=\operatorname{Fix}\left(J_{r}\right) \cap \operatorname{Fix}(S) \cap \operatorname{Fix}(G)=$ $F$. We observe that

$$
\begin{aligned}
\| x_{n}- & W x_{n} \| \\
= & \|\left(1-\theta_{1}-\theta_{2}\right)\left(x_{n}-J_{r} x_{n}\right) \\
& +\theta_{1}\left(x_{n}-S x_{n}\right)+\theta_{2}\left(x_{n}-G x_{n}\right) \| \\
\leq & \left(1-\theta_{1}-\theta_{2}\right)\left\|x_{n}-J_{r} x_{n}\right\| \\
& +\theta_{1}\left\|x_{n}-S x_{n}\right\|+\theta_{2}\left\|x_{n}-G x_{n}\right\| .
\end{aligned}
$$

From (82), (101), and (104), we obtain

$$
\lim _{n \rightarrow \infty}\left\|x_{n}-W x_{n}\right\|=0 .
$$

Now, we claim that

$$
\limsup _{n \rightarrow \infty}\left\langle f(q)-q, J\left(x_{n}-q\right)\right\rangle \leq 0,
$$

where $q=s-\lim _{t \rightarrow 0} x_{t}$ with $x_{t}$ being the fixed point of the contraction

$$
x \longmapsto t f(x)+(1-t) W x .
$$

Then $x_{t}$ solves the fixed point equation $x_{t}=t f\left(x_{t}\right)+(1-$ $t) W x_{t}$. Thus we have

$$
x_{t}-x_{n}=(1-t)\left(W x_{t}-x_{n}\right)+t\left(f\left(x_{t}\right)-x_{n}\right) .
$$

By Lemma 10, we conclude that

$$
\begin{aligned}
\left\|x_{t}-x_{n}\right\|^{2}= & \|(1-t)\left(W x_{t}-x_{n}\right) \\
& +t\left(f\left(x_{t}\right)-x_{n}\right) \|^{2} \\
\leq & (1-t)^{2}\left\|W x_{t}-x_{n}\right\|^{2} \\
& +2 t\left\langle f\left(x_{t}\right)-x_{n}, J\left(x_{t}-x_{n}\right)\right\rangle \\
\leq & (1-t)^{2}\left(\left\|W x_{t}-W x_{n}\right\|\right. \\
& \left.\quad+\left\|W x_{n}-x_{n}\right\|\right)^{2} \\
& +2 t\left\langle f\left(x_{t}\right)-x_{n}, J\left(x_{t}-x_{n}\right)\right\rangle \\
\leq & (1-t)^{2}\left(\left\|x_{t}-x_{n}\right\|\right. \\
& \left.\quad+\left\|W x_{n}-x_{n}\right\|\right)^{2} \\
& +2 t\left\langle f\left(x_{t}\right)-x_{n}, J\left(x_{t}-x_{n}\right)\right\rangle \\
= & (1-t)^{2}\left[\left\|x_{t}-x_{n}\right\|^{2}\right. \\
& \quad+2\left\|x_{t}-x_{n}\right\|\left\|W x_{n}-x_{n}\right\| \\
& \left.\quad+\left\|W x_{n}-x_{n}\right\|^{2}\right] \\
& +2 t\left\langle f\left(x_{t}\right)-x_{t}, J\left(x_{t}-x_{n}\right)\right\rangle \\
& +2 t\left\langle x_{t}-x_{n}, J\left(x_{t}-x_{n}\right)\right\rangle \\
= & \left(1-2 t+t^{2}\right)\left\|x_{t}-x_{n}\right\|^{2} \\
& +f_{n}(t)+2 t\left\langle f\left(x_{t}\right)-x_{t}, J\left(x_{t}-x_{n}\right)\right\rangle \\
& +22,
\end{aligned}
$$

where

$$
\begin{aligned}
f_{n}(t)= & (1-t)^{2}\left(2\left\|x_{t}-x_{n}\right\|+\left\|x_{n}-W x_{n}\right\|\right) \\
& \times\left\|x_{n}-W x_{n}\right\| \longrightarrow 0, \quad \text { as } n \longrightarrow \infty .
\end{aligned}
$$

It follows from (110) that

$$
\left\langle x_{t}-f\left(x_{t}\right), J\left(x_{t}-x_{n}\right)\right\rangle \leq \frac{t}{2}\left\|x_{t}-x_{n}\right\|^{2}+\frac{1}{2 t} f_{n}(t) .
$$

Letting $n \rightarrow \infty$ in (112) and noticing (111), we derive

$$
\limsup _{n \rightarrow \infty}\left\langle x_{t}-f\left(x_{t}\right), J\left(x_{t}-x_{n}\right)\right\rangle \leq \frac{t}{2} M_{2},
$$

where $M_{2}>0$ is a constant such that $\left\|x_{t}-x_{n}\right\|^{2} \leq M_{2}$ for all $t \in(0,1)$ and $n \geq 0$. Taking $t \rightarrow 0$ in (113), we have

$$
\limsup _{t \rightarrow 0} \limsup _{n \rightarrow \infty}\left\langle x_{t}-f\left(x_{t}\right), J\left(x_{t}-x_{n}\right)\right\rangle \leq 0 .
$$


On the other hand, we have

$$
\begin{aligned}
\left\langle f(q)-q, J\left(x_{n}-q\right)\right\rangle= & \left\langle f(q)-q, J\left(x_{n}-q\right)\right\rangle \\
& -\left\langle f(q)-q, J\left(x_{n}-x_{t}\right)\right\rangle \\
& +\left\langle f(q)-q, J\left(x_{n}-x_{t}\right)\right\rangle \\
& -\left\langle f(q)-x_{t}, J\left(x_{n}-x_{t}\right)\right\rangle \\
& +\left\langle f(q)-x_{t}, J\left(x_{n}-x_{t}\right)\right\rangle \\
& -\left\langle f\left(x_{t}\right)-x_{t}, J\left(x_{n}-x_{t}\right)\right\rangle \\
& +\left\langle f\left(x_{t}\right)-x_{t}, J\left(x_{n}-x_{t}\right)\right\rangle \\
= & \left\langle f(q)-q, J\left(x_{n}-q\right)-J\left(x_{n}-x_{t}\right)\right\rangle \\
& +\left\langle x_{t}-q, J\left(x_{n}-x_{t}\right)\right\rangle \\
& +\left\langle f(q)-f\left(x_{t}\right), J\left(x_{n}-x_{t}\right)\right\rangle \\
& +\left\langle f\left(x_{t}\right)-x_{t}, J\left(x_{n}-x_{t}\right)\right\rangle .
\end{aligned}
$$

It follows that

$$
\begin{aligned}
\limsup _{n \rightarrow \infty}\left\langle f(q)-q, J\left(x_{n}-q\right)\right\rangle \\
\leq \limsup _{n \rightarrow \infty}\left\langle f(q)-q, J\left(x_{n}-q\right)-J\left(x_{n}-x_{t}\right)\right\rangle \\
\quad+\left\|x_{t}-q\right\| \limsup _{n \rightarrow \infty}\left\|x_{n}-x_{t}\right\| \\
\quad+\rho\left\|q-x_{t}\right\| \limsup _{n \rightarrow \infty}\left\|x_{n}-x_{t}\right\| \\
\quad+\limsup _{n \rightarrow \infty}\left\langle f\left(x_{t}\right)-x_{t}, J\left(x_{n}-x_{t}\right)\right\rangle .
\end{aligned}
$$

Taking into account that $x_{t} \rightarrow q$ as $t \rightarrow 0$, we have

$$
\begin{gathered}
\limsup _{n \rightarrow \infty}\left\langle f(q)-q, J\left(x_{n}-q\right)\right\rangle \\
=\limsup _{t \rightarrow 0} \limsup _{n \rightarrow \infty}\left\langle f(q)-q, J\left(x_{n}-q\right)\right\rangle \\
\leq \limsup _{t \rightarrow 0} \limsup _{n \rightarrow \infty}\left\langle f(q)-q, J\left(x_{n}-q\right)\right. \\
\left.-J\left(x_{n}-x_{t}\right)\right\rangle .
\end{gathered}
$$

Since $X$ has a uniformly Gateaux differentiable norm, the duality mapping $J$ is norm-to-weak* uniformly continuous on bounded subsets of $X$. Consequently, the two limits are interchangeable and hence (107) holds. From (82), we get $\left(y_{n}-q\right)-\left(x_{n}-q\right) \rightarrow 0$. Noticing the norm-to-weak ${ }^{*}$ uniform continuity of $J$ on bounded subsets of $X$, we deduce from (107) that

$$
\begin{aligned}
& \limsup _{n \rightarrow \infty}\left\langle f(q)-q, J\left(y_{n}-q\right)\right\rangle \\
&=\limsup _{n \rightarrow \infty}\left(\left\langle f(q)-q, J\left(x_{n}-q\right)\right\rangle\right.\left.\quad+\left\langle f(q)-q, J\left(y_{n}-q\right)-J\left(x_{n}-q\right)\right\rangle\right) \\
& \\
&=\limsup _{n \rightarrow \infty}\left\langle f(q)-q, J\left(x_{n}-q\right)\right\rangle \leq 0 .
\end{aligned}
$$

Finally, let us show that $x_{n} \rightarrow q$ as $n \rightarrow \infty$. Indeed, observe that

$$
\begin{aligned}
\left\|y_{n}-q\right\|^{2}= & \| \alpha_{n}\left(f\left(x_{n}\right)-f(q)\right) \\
& +\beta_{n}\left(x_{n}-q\right)+\gamma_{n}\left(J_{r_{n}} x_{n}-q\right) \\
& +\delta_{n}\left(S_{n} y_{n}-q\right)+\alpha_{n}(f(q)-q) \|^{2} \\
\leq & \| \alpha_{n}\left(f\left(x_{n}\right)-f(q)\right) \\
& +\beta_{n}\left(x_{n}-q\right)+\gamma_{n}\left(J_{r_{n}} x_{n}-q\right) \\
& +\delta_{n}\left(S_{n} y_{n}-q\right) \|^{2} \\
& +2 \alpha_{n}\left\langle f(q)-q, J\left(y_{n}-q\right)\right\rangle \\
\leq & \alpha_{n}\left\|f\left(x_{n}\right)-f(q)\right\|^{2} \\
& +\beta_{n}\left\|x_{n}-q\right\|^{2}+\gamma_{n}\left\|J_{r_{n}} x_{n}-q\right\|^{2} \\
& +\delta_{n}\left\|S_{n} y_{n}-q\right\|^{2} \\
& +2 \alpha_{n}\left\langle f(q)-q, J\left(y_{n}-q\right)\right\rangle \\
\leq & \alpha_{n} \rho\left\|x_{n}-q\right\|^{2} \\
& +\beta_{n}\left\|x_{n}-q\right\|^{2}+\gamma_{n}\left\|x_{n}-q\right\| \\
& +\delta_{n}\left\|y_{n}-q\right\|^{2} \\
& +2 \alpha_{n}\left\langle f(q)-q, J\left(y_{n}-q\right)\right\rangle \\
= & \left(1-\delta_{n}-\alpha_{n}(1-\rho)\right)\left\|x_{n}-q\right\|^{2} \\
& +\delta_{n}\left\|y_{n}-q\right\|^{2}\left\langle f(q)-q, J\left(y_{n}-q\right)\right\rangle, \\
& 2 \alpha_{n}\langle f(f)
\end{aligned}
$$

which implies that

$$
\begin{aligned}
\left\|y_{n}-q\right\|^{2} \leq & \left(1-\frac{\alpha_{n}(1-\rho)}{1-\delta_{n}}\right)\left\|x_{n}-q\right\|^{2} \\
& +\frac{2 \alpha_{n}}{1-\delta_{n}}\left\langle f(q)-q, J\left(y_{n}-q\right)\right\rangle .
\end{aligned}
$$


Thus, we have

$$
\begin{aligned}
\left\|x_{n+1}-q\right\|^{2} \leq & \sigma_{n}\left\|y_{n}-q\right\|^{2} \\
& +\left(1-\sigma_{n}\right)\left\|G y_{n}-q\right\|^{2} \leq\left\|y_{n}-q\right\|^{2} \\
\leq & \left(1-\frac{\alpha_{n}(1-\rho)}{1-\delta_{n}}\right)\left\|x_{n}-q\right\|^{2} \\
& +\frac{2 \alpha_{n}}{1-\delta_{n}}\left\langle f(q)-q, J\left(y_{n}-q\right)\right\rangle \\
\leq & \left(1-\frac{\alpha_{n}(1-\rho)}{1-\delta_{n}}\right)\left\|x_{n}-q\right\|^{2} \\
& +\frac{\alpha_{n}(1-\rho)}{1-\delta_{n}} \frac{2\left\langle f(q)-q, J\left(y_{n}-q\right)\right\rangle}{1-\rho} .
\end{aligned}
$$

Applying Lemma 9 to (121), we conclude from condition (i) and (118) that $x_{n} \rightarrow q$ as $n \rightarrow \infty$. This completes the proof.

The following results can be obtained from Theorem 23 . We, therefore, omit the proof.

Corollary 24. Let $C$ be a nonempty closed convex subset of a uniformly convex Banach space $X$ which has a uniformly Gateaux differentiable norm. Let $\Pi_{C}$ be a sunny nonexpansive retraction from $X$ onto $C$. Let $A \subset X \times X$ be an accretive operator in $X$ such that $\overline{D(A)} \subset C \subset \bigcap_{r>0} R(I+r A)$. Let $B_{i}: C \rightarrow X$ be $\zeta_{i}$ strictly pseudocontractive and $\theta_{i}$ strongly accretive with $\theta_{i}+\zeta_{i} \geq 1$ for each $i=1,2$. Define the mapping $G: C \rightarrow C$ by $G=\Pi_{C}\left(I-\mu_{1} B_{1}\right) \Pi_{C}\left(I-\mu_{2} B_{2}\right)$ where $1-\left(\zeta_{i} /\left(1+\zeta_{i}\right)\right)\left(1-\sqrt{\left(1-\theta_{i}\right) / \zeta_{i}}\right) \leq \mu_{i} \leq 1$ for $i=1,2$. Let $f: C \rightarrow C$ be a contraction with coefficient $\rho \in(0,1)$. Let $S: C \rightarrow C$ be a nonexpansive mapping such that $F=$ $\operatorname{Fix}(S) \cap \Omega \cap A^{-1} 0 \neq \emptyset$ where $\Omega=\operatorname{Fix}(G)$. For arbitrarily given $x_{0} \in C$, let $\left\{x_{n}\right\}$ be the sequence generated by

$$
\begin{aligned}
y_{n}=\alpha_{n} f & \left(x_{n}\right)+\beta_{n} x_{n}+\gamma_{n} J_{r_{n}} x_{n}+\delta_{n} S y_{n}, \\
x_{n+1}= & \sigma_{n} y_{n}+\left(1-\sigma_{n}\right) \Pi_{C}\left(I-\mu_{1} B_{1}\right) \\
& \times \Pi_{C}\left(I-\mu_{2} B_{2}\right) y_{n}, \quad \forall n \geq 0 .
\end{aligned}
$$

Suppose that $\left\{r_{n}\right\} \subset(0, \infty),\left\{\sigma_{n}\right\},\left\{\alpha_{n}\right\},\left\{\beta_{n}\right\},\left\{\gamma_{n}\right\},\left\{\delta_{n}\right\} \subset[0,1]$, $\alpha_{n}+\beta_{n}+\gamma_{n}+\delta_{n}=1$, and the following conditions hold:

(i) $\lim _{n \rightarrow \infty} \alpha_{n}=0$ and $\sum_{n=0}^{\infty} \alpha_{n}=\infty$;

(ii) $\left\{\gamma_{n}\right\},\left\{\delta_{n}\right\} \subset[c, d]$ for some $c, d \in(0,1)$;

(iii) $\sum_{n=1}^{\infty}\left(\left|\sigma_{n}-\sigma_{n-1}\right|+\left|\alpha_{n}-\alpha_{n-1}\right|+\left|\beta_{n}-\beta_{n-1}\right|+\left|\gamma_{n}-\gamma_{n-1}\right|+\right.$ $\left.\left|\delta_{n}-\delta_{n-1}\right|\right)<\infty$

(iv) $\sum_{n=1}^{\infty}\left|r_{n}-r_{n-1}\right|<\infty$ and $r_{n} \geq \varepsilon>0$, for all $n \geq 0$ for some $\varepsilon>0$;

(v) $0<\liminf _{n \rightarrow \infty} \beta_{n} \leq \lim \sup _{n \rightarrow \infty} \beta_{n}<1$ and $0<$ $\liminf _{n \rightarrow \infty} \sigma_{n} \leq \limsup _{n \rightarrow \infty} \sigma_{n}<1$.

Then $\left\{x_{n}\right\}$ converges strongly to $q \in F$, which solves the following VIP:

$$
\langle q-f(q), J(q-p)\rangle \leq 0, \quad \forall p \in F
$$

Corollary 25. Let $C$ be a nonempty closed convex subset of a uniformly convex Banach space X which has a uniformly Gateaux differentiable norm. Let $\Pi_{C}$ be a sunny nonexpansive retraction from $X$ onto $C$. Let $A \subset X \times X$ be an accretive operator in $X$ such that $\overline{D(A)} \subset C \subset \bigcap_{r>0} R(I+r A)$. Let $f: C \rightarrow C$ be a contraction with coefficient $\rho \in(0,1)$. Let $V: C \rightarrow C$ be a self-mapping such that $I-V: C \rightarrow X$ is $\zeta$ strictly pseudocontractive and $\theta$ strongly accretive with $\theta+\zeta \geq$ 1. Let $\left\{S_{i}\right\}_{i=0}^{\infty}$ be an infinite family of nonexpansive mappings of $C$ into itself such that $F=\bigcap_{i=0}^{\infty} \operatorname{Fix}\left(S_{i}\right) \cap \operatorname{Fix}(V) \cap A^{-1} 0 \neq \emptyset$. For arbitrarily given $x_{0} \in C$, let $\left\{x_{n}\right\}$ be the sequence generated by

$$
\begin{array}{rl}
y_{n}=\alpha_{n} & f\left(x_{n}\right)+\beta_{n} x_{n}+\gamma_{n} J_{r_{n}} x_{n}+\delta_{n} S_{n} y_{n}, \\
x_{n+1}= & \sigma_{n} y_{n}+\left(1-\sigma_{n}\right) \\
& \times((1-\alpha) I+\alpha V) y_{n}, \quad \forall n \geq 0,
\end{array}
$$

where $1-(\zeta /(1+\zeta))(1-\sqrt{(1-\theta) / \zeta}) \leq \alpha \leq 1$. Suppose that $\left\{r_{n}\right\} \subset(0, \infty),\left\{\sigma_{n}\right\},\left\{\alpha_{n}\right\},\left\{\beta_{n}\right\},\left\{\gamma_{n}\right\},\left\{\delta_{n}\right\} \subset[0,1], \alpha_{n}+\beta_{n}+\gamma_{n}+$ $\delta_{n}=1$, and the following conditions hold:

(i) $\lim _{n \rightarrow \infty} \alpha_{n}=0$ and $\sum_{n=0}^{\infty} \alpha_{n}=\infty$;

(ii) $\left\{\gamma_{n}\right\},\left\{\delta_{n}\right\} \subset[c, d]$ for some $c, d \in(0,1)$;

(iii) $\sum_{n=1}^{\infty}\left(\left|\sigma_{n}-\sigma_{n-1}\right|+\left|\alpha_{n}-\alpha_{n-1}\right|+\left|\beta_{n}-\beta_{n-1}\right|+\left|\gamma_{n}-\gamma_{n-1}\right|+\right.$ $\left.\left|\delta_{n}-\delta_{n-1}\right|\right)<\infty$;

(iv) $\sum_{n=1}^{\infty}\left|r_{n}-r_{n-1}\right|<\infty$ and $r_{n} \geq \varepsilon>0$, for all $n \geq 0$ for some $\varepsilon>0$

(v) $0<\liminf _{n \rightarrow \infty} \beta_{n} \leq \lim \sup _{n \rightarrow \infty} \beta_{n}<1$ and $0<$ $\liminf _{n \rightarrow \infty} \sigma_{n} \leq \lim \sup _{n \rightarrow \infty} \sigma_{n}<1$.

Assume that $\sum_{n=0}^{\infty} \sup _{x \in D}\left\|S_{n+1} x-S_{n} x\right\|<\infty$ for any bounded subset $D$ of $C$ and let $S$ be a mapping of $C$ into itself defined by $S x=\lim _{n \rightarrow \infty} S_{n} x$ for all $x \in C$ and suppose that $\operatorname{Fix}(S)=$ $\bigcap_{i=0}^{\infty} \operatorname{Fix}\left(S_{i}\right)$. Then $\left\{x_{n}\right\}$ converges strongly to $q \in F$, which solves the following VIP:

$$
\langle q-f(q), J(q-p)\rangle \leq 0, \quad \forall p \in F .
$$

Proof. In Theorem 23, we put $B_{1}=I-V, B_{2}=0$, and $\mu_{1}=\alpha$ where $1-(\zeta /(1+\zeta))(1-\sqrt{(1-\theta) / \zeta}) \leq \alpha \leq 1$. Then GSVI (14) is equivalent to the VIP of finding $x^{*} \in C$ such that

$$
\left\langle B_{1} x^{*}, J\left(x-x^{*}\right)\right\rangle \geq 0, \quad \forall x \in C .
$$


In this case, $B_{1}: C \rightarrow X$ is $\zeta$ strictly pseudocontractive and $\theta$ strongly accretive. It is not hard to see that $\operatorname{Fix}(V)=\mathrm{VI}(C$, $\left.B_{1}\right)$. As a matter of fact, we have, for $\alpha>0$,

$$
\begin{aligned}
u \in \mathrm{VI}\left(C, B_{1}\right) & \Longleftrightarrow\left\langle B_{1} u, J(y-u)\right\rangle \geq 0, \quad \forall y \in C \\
& \Longleftrightarrow\left\langle u-\alpha B_{1} u-u, J(u-y)\right\rangle \geq 0, \\
& \forall y \in C \\
& \Longleftrightarrow u=\Pi_{C}\left(u-\alpha B_{1} u\right) \\
& \Longleftrightarrow u=\Pi_{C}(u-\alpha u+\alpha V u) \\
& \Longleftrightarrow\langle u-\alpha u+\alpha V u-u, J(u-y)\rangle \geq 0, \quad \forall y \in C \\
& \Longleftrightarrow\langle u-V u, J(u-y)\rangle \leq 0, \quad \forall y \in C \\
& \Longleftrightarrow u=V u \\
& \Longleftrightarrow u \in \operatorname{Fix}(V) .
\end{aligned}
$$

Accordingly, we know that $F=\bigcap_{i=1}^{\infty} \operatorname{Fix}\left(S_{i}\right) \cap \Omega \cap A^{-1} 0=$ $\bigcap_{i=0}^{\infty} \operatorname{Fix}\left(S_{i}\right) \cap \operatorname{Fix}(V) \cap A^{-1} 0$, and

$$
\begin{aligned}
\Pi_{C}\left(I-\mu_{1} B_{1}\right) \Pi_{C}\left(I-\mu_{2} B_{2}\right) y_{n} \\
=\Pi_{C}\left(I-\mu_{1} B_{1}\right) y_{n} \\
=\Pi_{C}\left((1-\alpha) y_{n}+\alpha V y_{n}\right) \\
=(1-\alpha) y_{n}+\alpha V y_{n} .
\end{aligned}
$$

So, the scheme (53) reduces to (124). Therefore, the desired result follows from Theorem 23 .

Corollary 26. Let $C$ be a nonempty closed convex subset of a uniformly convex Banach space $X$ which has a uniformly Gateaux differentiable norm. Let $\Pi_{C}$ be a sunny nonexpansive retraction from $X$ onto $C$. Let $A \subset X \times X$ be an accretive operator in $X$ such that $\overline{D(A)} \subset C \subset \bigcap_{r>0} R(I+r A)$. Let $f: C \rightarrow C$ be a contraction with coefficient $\rho \in(0,1)$. Let $V: C \rightarrow C$ be a self-mapping such that $I-V: C \rightarrow X$ is $\zeta$ strictly pseudocontractive and $\theta$ strongly accretive with $\theta+\zeta \geq 1$. Let $S: C \rightarrow C$ be a nonexpansive mapping such that $F=\operatorname{Fix}(S) \cap \operatorname{Fix}(V) \cap A^{-1} 0 \neq \emptyset$. For arbitrarily given $x_{0} \in C$, let $\left\{x_{n}\right\}$ be the sequence generated by

$$
\begin{gathered}
y_{n}=\alpha_{n} f\left(x_{n}\right)+\beta_{n} x_{n}+\gamma_{n} J_{r_{n}} x_{n}+\delta_{n} S y_{n}, \\
x_{n+1}=\sigma_{n} y_{n}+\left(1-\sigma_{n}\right)((1-\alpha) I+\alpha V) y_{n}, \quad \forall n \geq 0,
\end{gathered}
$$

where $1-(\zeta /(1+\zeta))(1-\sqrt{(1-\theta) / \zeta}) \leq \alpha \leq 1$. Suppose that $\left\{r_{n}\right\} \subset(0, \infty),\left\{\sigma_{n}\right\},\left\{\alpha_{n}\right\},\left\{\beta_{n}\right\},\left\{\gamma_{n}\right\},\left\{\delta_{n}\right\} \subset[0,1], \alpha_{n}+\beta_{n}+\gamma_{n}+$ $\delta_{n}=1$, and the following conditions hold:

(i) $\lim _{n \rightarrow \infty} \alpha_{n}=0$ and $\sum_{n=0}^{\infty} \alpha_{n}=\infty$;

(ii) $\left\{\gamma_{n}\right\},\left\{\delta_{n}\right\} \subset[c, d]$ for some $c, d \in(0,1)$; (iii) $\sum_{n=1}^{\infty}\left(\left|\sigma_{n}-\sigma_{n-1}\right|+\left|\alpha_{n}-\alpha_{n-1}\right|+\left|\beta_{n}-\beta_{n-1}\right|+\left|\gamma_{n}-\gamma_{n-1}\right|+\right.$ $\left.\left|\delta_{n}-\delta_{n-1}\right|\right)<\infty$;

(iv) $\sum_{n=1}^{\infty}\left|r_{n}-r_{n-1}\right|<\infty$ and $r_{n} \geq \varepsilon>0$, for all $n \geq 0$ for some $\varepsilon>0$;

(v) $0<\liminf _{n \rightarrow \infty} \beta_{n} \leq \limsup _{n \rightarrow \infty} \beta_{n}<1$ and $0<$ $\liminf _{n \rightarrow \infty} \sigma_{n} \leq \limsup _{n \rightarrow \infty} \sigma_{n}<1$.

Then $\left\{x_{n}\right\}$ converges strongly to $q \in F$, which solves the following VIP:

$$
\langle q-f(q), J(q-p)\rangle \leq 0, \quad \forall p \in F .
$$

Remark 27. Our Theorem 23 improves, extends, supplements, and develops $\mathrm{Cai}$ and $\mathrm{Bu}$ [10, Theorem 3.1 and Corollary 3.2] and Jung [5, Theorems 3.1] in the following aspects.

(i) The problem of finding a point $q \in \bigcap_{n} \operatorname{Fix}\left(S_{n}\right) \cap \Omega \cap$ $A^{-1} 0$ in our Theorem 23 is more general and more subtle than every one of both the problem of finding a point $q \epsilon$ $\bigcap_{n} \operatorname{Fix}\left(S_{n}\right) \cap \Omega$ in Cai and $\mathrm{Bu}[10$, Theorem 3.1] and the problem of finding a point $q \in A^{-1} 0$ in Jung [5, Theorem 3.1].

(ii) Our Theorem 23 drops the assumption of the asymptotical regularity of $\left\{x_{n}\right\}$ in [5, Theorems 3.1] (i.e., $\left.\lim _{n \rightarrow \infty}\left\|x_{n}-x_{n+1}\right\|=0\right)$.

(iii) Cai and Bu's proof in [10, Theorem 3.1] depends on the argument techniques in [13], inequality (21) in 2uniformly smooth Banach spaces, and inequality (27) in smooth and uniform convex Banach spaces. Jung's proof in [5, Theorem 3.1] depends on the resolvent identity in Proposition 1. It is worth emphasizing that the proof of our Theorem 23 does not depend on the argument techniques in [13], inequality (21) in 2-uniformly smooth Banach spaces, and inequality (27) in smooth and uniform convex Banach spaces. However, it depends on the resolvent identity in Proposition 1 and the inequalities in uniform convex Banach spaces; see Lemmas 13 and 17 in Section 2 of this paper.

(iv) The assumption of the uniformly convex and 2uniformly smooth Banach space $X$ in [10, Theorem 3.1] is weakened to the one of the uniformly convex Banach space $X$ having a uniformly Gateaux differentiable norm in our Theorem 23

(v) The iterative scheme in our Theorem 23 is very different from every one in both [10, Theorem 3.1] and [5, Theorem 3.1] because the first iteration step in our iterative scheme is implicit.

(vi) The problem of finding a point $q \in \operatorname{Fix}(S) \cap \Omega$ in $[10$, Corollary 3.2] is extended to develop the problem of finding a point $q \in \operatorname{Fix}(S) \cap \Omega \cap A^{-1} 0$ in our Corollary 24 .

\section{Hybrid Explicit Extragradient Algorithm}

In this section, let $C$ be a nonempty closed convex subset of a uniformly convex Banach space $X$ which has a uniformly Gateaux differentiable norm. We suggest and analyze a hybrid explicit extragradient algorithm for finding a zero of $A \subset X \times$ $X$ an accretive operator in $X$ with $\overline{D(A)} \subset C \subset \bigcap_{r>0} R(I+$ $r A)$ and solving a general system of variational inequalities and a common fixed point problem of an infinite family of nonexpansive self-mappings in $X$. 
Theorem 28. Let $C$ be a nonempty closed convex subset of a uniformly convex Banach space $X$ which has a uniformly Gateaux differentiable norm. Let $\Pi_{C}$ be a sunny nonexpansive retraction from $X$ onto $C$. Let $A \subset X \times X$ be an accretive operator in $X$ such that $\overline{D(A)} \subset C \subset \bigcap_{r>0} R(I+r A)$. Let $B_{i}: C \rightarrow X$ be $\zeta_{i}$ strictly pseudocontractive and $\theta_{i}$ strongly accretive with $\theta_{i}+\zeta_{i} \geq 1$ for each $i=1,2$. Define the mapping $G: C \rightarrow C$ by $G=\Pi_{C}\left(I-\mu_{1} B_{1}\right) \Pi_{C}\left(I-\mu_{2} B_{2}\right)$ where $1-\left(\zeta_{i} /\left(1+\zeta_{i}\right)\right)\left(1-\sqrt{\left(1-\theta_{i}\right) / \zeta_{i}}\right) \leq \mu_{i} \leq 1$ for $i=1,2$. Let $f: C \rightarrow C$ be a contraction with coefficient $\rho \in(0,1)$. Let $\left\{S_{i}\right\}_{i=0}^{\infty}$ be an infinite family of nonexpansive mappings of $C$ into itself such that $F=\bigcap_{i=0}^{\infty} \operatorname{Fix}\left(S_{i}\right) \cap \Omega \cap A^{-1} 0 \neq \emptyset$ where $\Omega=\operatorname{Fix}(G)$. For arbitrarily given $x_{0} \in C$, let $\left\{x_{n}\right\}$ be the sequence generated by

$$
\begin{gathered}
y_{n}=\alpha_{n} f\left(x_{n}\right)+\beta_{n} x_{n}+\gamma_{n} J_{r_{n}} x_{n}+\delta_{n} S_{n} x_{n}, \\
x_{n+1}=\sigma_{n} y_{n}+\left(1-\sigma_{n}\right) \Pi_{C}\left(I-\mu_{1} B_{1}\right) \\
\times \Pi_{C}\left(I-\mu_{2} B_{2}\right) y_{n}, \quad \forall n \geq 0 .
\end{gathered}
$$

Suppose that $\left\{r_{n}\right\} \subset(0, \infty),\left\{\sigma_{n}\right\},\left\{\alpha_{n}\right\},\left\{\beta_{n}\right\},\left\{\gamma_{n}\right\},\left\{\delta_{n}\right\} \subset[0,1]$, $\alpha_{n}+\beta_{n}+\gamma_{n}+\delta_{n}=1$, and the following conditions hold:

(i) $\lim _{n \rightarrow \infty} \alpha_{n}=0$ and $\sum_{n=0}^{\infty} \alpha_{n}=\infty$;

(ii) $\left\{\gamma_{n}\right\},\left\{\delta_{n}\right\} \subset[c, d]$ for some $c, d \in(0,1)$;

(iii) $\sum_{n=1}^{\infty}\left(\left|\sigma_{n}-\sigma_{n-1}\right|+\left|\alpha_{n}-\alpha_{n-1}\right|+\left|\beta_{n}-\beta_{n-1}\right|+\left|\gamma_{n}-\gamma_{n-1}\right|+\right.$ $\left.\left|\delta_{n}-\delta_{n-1}\right|\right)<\infty$;

(iv) $\sum_{n=1}^{\infty}\left|r_{n}-r_{n-1}\right|<\infty$ and $r_{n} \geq \varepsilon>0$, for all $n \geq 0$ for some $\varepsilon>0$;

(v) $0<\liminf _{n \rightarrow \infty} \beta_{n} \leq \limsup _{n \rightarrow \infty} \beta_{n}<1$ and $0<$ $\liminf _{n \rightarrow \infty} \sigma_{n} \leq \limsup _{n \rightarrow \infty} \sigma_{n}<1$.

Assume that $\sum_{n=0}^{\infty} \sup _{x \in D}\left\|S_{n+1} x-S_{n} x\right\|<\infty$ for any bounded subset $D$ of $C$ and let $S$ be a mapping of $C$ into itself defined by $S x=\lim _{n \rightarrow \infty} S_{n} x$ for all $x \in C$ and suppose that $\operatorname{Fix}(S)=$ $\bigcap_{i=0}^{\infty} \operatorname{Fix}\left(S_{i}\right)$. Then $\left\{x_{n}\right\}$ converges strongly to $q \in F$, which solves the following VIP:

$$
\langle q-f(q), J(q-p)\rangle \leq 0, \quad \forall p \in F .
$$

Proof. It is easy to see that (131) can be rewritten as follows

$$
\begin{gathered}
y_{n}=\alpha_{n} f\left(x_{n}\right)+\beta_{n} x_{n}+\gamma_{n} J_{r_{n}} x_{n}+\delta_{n} S_{n} x_{n}, \\
x_{n+1}=\sigma_{n} y_{n}+\left(1-\sigma_{n}\right) G y_{n}, \quad \forall n \geq 0,
\end{gathered}
$$

where $G=\Pi_{C}\left(I-\mu_{1} B_{1}\right) \Pi_{C}\left(I-\mu_{2} B_{2}\right)$. By Lemma 20 we know that $G$ is a nonexpansive self-mapping on $C$.
Now, let us show that the sequence $\left\{x_{n}\right\}$ is bounded. Indeed, take a fixed $p \in F$ arbitrarily. Then from (133), we have

$$
\begin{aligned}
\left\|y_{n}-p\right\| \leq & \alpha_{n}\left\|f\left(x_{n}\right)-p\right\|+\beta_{n}\left\|x_{n}-p\right\| \\
& +\gamma_{n}\left\|J_{r_{n}} x_{n}-p\right\|+\delta_{n}\left\|S_{n} x_{n}-p\right\| \\
\leq & \alpha_{n}\left(\left\|f\left(x_{n}\right)-f(p)\right\|+\|f(p)-p\|\right) \\
& +\beta_{n}\left\|x_{n}-p\right\| \\
& +\gamma_{n}\left\|x_{n}-p\right\|+\delta_{n}\left\|x_{n}-p\right\| \\
\leq & \alpha_{n}\left(\rho\left\|x_{n}-p\right\|+\|f(p)-p\|\right) \\
& +\left(1-\alpha_{n}\right)\left\|x_{n}-p\right\| \\
= & \left(1-(1-\rho) \alpha_{n}\right)\left\|x_{n}-p\right\| \\
& +\alpha_{n}\|f(p)-p\| \\
= & \left(1-(1-\rho) \alpha_{n}\right)\left\|x_{n}-p\right\| \\
& +(1-\rho) \alpha_{n} \frac{\|f(p)-p\|}{1-\rho},
\end{aligned}
$$

and hence

$$
\begin{aligned}
\left\|x_{n+1}-p\right\| \leq & \sigma_{n}\left\|y_{n}-p\right\| \\
& +\left(1-\sigma_{n}\right)\left\|G y_{n}-p\right\| \\
\leq & \left\|y_{n}-p\right\| \\
\leq & \left(1-(1-\rho) \alpha_{n}\right)\left\|x_{n}-p\right\| \\
& +(1-\rho) \alpha_{n} \frac{\|f(p)-p\|}{1-\rho} \\
\leq & \max \left\{\left\|x_{n}-p\right\|, \frac{\|f(p)-p\|}{1-\rho}\right\} .
\end{aligned}
$$

By induction, we obtain

$$
\left\|x_{n}-p\right\| \leq \max \left\{\left\|x_{0}-p\right\|, \frac{\|f(p)-p\|}{1-\rho}\right\}, \quad \forall n \geq 0
$$

It immediately follows that $\left\{x_{n}\right\}$ is bounded and so are $\left\{y_{n}\right\}$, $\left\{G y_{n}\right\},\left\{f\left(x_{n}\right)\right\},\left\{J_{r_{n}} x_{n}\right\}$, and $\left\{S_{n} x_{n}\right\}$.

Let us show that

$$
\lim _{n \rightarrow \infty}\left\|x_{n+1}-x_{n}\right\|=0
$$

As a matter of fact, observe that $y_{n}$ can be rewritten as follows:

$$
y_{n}=\beta_{n} x_{n}+\left(1-\beta_{n}\right) z_{n}
$$


where $z_{n}=\left(\alpha_{n} f\left(x_{n}\right)+\gamma_{n} J_{r_{n}} x_{n}+\delta_{n} S_{n} x_{n}\right) /\left(1-\beta_{n}\right)$. Observe that

$$
\begin{aligned}
& \left\|z_{n}-z_{n-1}\right\|=\| \frac{\alpha_{n} f\left(x_{n}\right)+\gamma_{n} J_{r_{n}} x_{n}+\delta_{n} S_{n} x_{n}}{1-\beta_{n}} \\
& -\frac{\alpha_{n-1} f\left(x_{n-1}\right)+\gamma_{n-1} J_{r_{n-1}} x_{n-1}+\delta_{n-1} S_{n-1} x_{n-1}}{1-\beta_{n-1}} \| \\
& =\left\|\frac{y_{n}-\beta_{n} x_{n}}{1-\beta_{n}}-\frac{y_{n-1}-\beta_{n-1} x_{n-1}}{1-\beta_{n-1}}\right\| \\
& =\| \frac{y_{n}-\beta_{n} x_{n}}{1-\beta_{n}}-\frac{y_{n-1}-\beta_{n-1} x_{n-1}}{1-\beta_{n}} \\
& +\frac{y_{n-1}-\beta_{n-1} x_{n-1}}{1-\beta_{n}}-\frac{y_{n-1}-\beta_{n-1} x_{n-1}}{1-\beta_{n-1}} \| \\
& \leq\left\|\frac{y_{n}-\beta_{n} x_{n}}{1-\beta_{n}}-\frac{y_{n-1}-\beta_{n-1} x_{n-1}}{1-\beta_{n}}\right\| \\
& +\left\|\frac{y_{n-1}-\beta_{n-1} x_{n-1}}{1-\beta_{n}}-\frac{y_{n-1}-\beta_{n-1} x_{n-1}}{1-\beta_{n-1}}\right\| \\
& =\frac{1}{1-\beta_{n}}\left\|y_{n}-\beta_{n} x_{n}-\left(y_{n-1}-\beta_{n-1} x_{n-1}\right)\right\| \\
& +\left|\frac{1}{1-\beta_{n}}-\frac{1}{1-\beta_{n-1}}\right|\left\|y_{n-1}-\beta_{n-1} x_{n-1}\right\| \\
& =\frac{1}{1-\beta_{n}}\left\|y_{n}-\beta_{n} x_{n}-\left(y_{n-1}-\beta_{n-1} x_{n-1}\right)\right\| \\
& +\frac{\left|\beta_{n}-\beta_{n-1}\right|}{\left(1-\beta_{n-1}\right)\left(1-\beta_{n}\right)}\left\|y_{n-1}-\beta_{n-1} x_{n-1}\right\| \\
& =\frac{1}{1-\beta_{n}} \| \alpha_{n} f\left(x_{n}\right)+\gamma_{n} J_{r_{n}} x_{n} \\
& +\delta_{n} S_{n} x_{n}-\alpha_{n-1} f\left(x_{n-1}\right) \\
& -\gamma_{n-1} J_{r_{n-1}} x_{n-1}-\delta_{n-1} S_{n-1} x_{n-1} \| \\
& +\frac{\left|\beta_{n}-\beta_{n-1}\right|}{\left(1-\beta_{n-1}\right)\left(1-\beta_{n}\right)}\left\|y_{n-1}-\beta_{n-1} x_{n-1}\right\| \\
& \leq \frac{1}{1-\beta_{n}}\left[\alpha_{n}\left\|f\left(x_{n}\right)-f\left(x_{n-1}\right)\right\|\right. \\
& +\gamma_{n}\left\|J_{r_{n}} x_{n}-J_{r_{n-1}} x_{n-1}\right\| \\
& +\delta_{n}\left\|S_{n} x_{n}-S_{n-1} x_{n-1}\right\| \\
& +\left|\alpha_{n}-\alpha_{n-1}\right|\left\|f\left(x_{n-1}\right)\right\| \\
& +\left|\gamma_{n}-\gamma_{n-1}\right|\left\|J_{r_{n-1}} x_{n-1}\right\| \\
& \left.+\left|\delta_{n}-\delta_{n-1}\right|\left\|S_{n-1} x_{n-1}\right\|\right] \\
& +\frac{\left|\beta_{n}-\beta_{n-1}\right|}{\left(1-\beta_{n-1}\right)\left(1-\beta_{n}\right)}\left\|y_{n-1}-\beta_{n-1} x_{n-1}\right\| \text {. }
\end{aligned}
$$

On the other hand, repeating the same arguments as those of (66) in the proof of Theorem 23, we can derive

$$
\begin{aligned}
\left\|J_{r_{n}} x_{n}-J_{r_{n-1}} x_{n-1}\right\| \leq & \left\|x_{n-1}-x_{n}\right\| \\
& +M_{0}\left|r_{n-1}-r_{n}\right|, \quad \forall n \geq 1,
\end{aligned}
$$

where $\sup _{n \geq 1}\left\{(1 / \varepsilon)\left(\left\|J_{r_{n}} x_{n}-x_{n-1}\right\|+\left\|J_{r_{n-1}} x_{n-1}-x_{n}\right\|\right)\right\} \leq M_{0}$ for some $M_{0}>0$. Substituting (140) into (139), we have

$$
\begin{aligned}
& \left\|z_{n}-z_{n-1}\right\| \leq \frac{1}{1-\beta_{n}}\left[\alpha_{n}\left\|f\left(x_{n}\right)-f\left(x_{n-1}\right)\right\|\right. \\
& +\gamma_{n}\left(\left\|x_{n-1}-x_{n}\right\|+M_{0}\left|r_{n-1}-r_{n}\right|\right) \\
& +\delta_{n}\left\|S_{n} x_{n}-S_{n-1} x_{n-1}\right\| \\
& +\left|\alpha_{n}-\alpha_{n-1}\right|\left\|f\left(x_{n-1}\right)\right\| \\
& +\left|\gamma_{n}-\gamma_{n-1}\right|\left\|J_{r_{n-1}} x_{n-1}\right\| \\
& \left.+\left|\delta_{n}-\delta_{n-1}\right|\left\|S_{n-1} x_{n-1}\right\|\right] \\
& +\frac{\left|\beta_{n}-\beta_{n-1}\right|}{\left(1-\beta_{n-1}\right)\left(1-\beta_{n}\right)}\left\|y_{n-1}-\beta_{n-1} x_{n-1}\right\| \\
& \leq \frac{1}{1-\beta_{n}}\left[\alpha_{n} \rho\left\|x_{n}-x_{n-1}\right\|\right. \\
& +\gamma_{n}\left(\left\|x_{n-1}-x_{n}\right\|+M_{0}\left|r_{n-1}-r_{n}\right|\right) \\
& +\delta_{n}\left\|S_{n} x_{n}-S_{n} x_{n-1}\right\| \\
& +\left|\alpha_{n}-\alpha_{n-1}\right|\left\|f\left(x_{n-1}\right)\right\| \\
& +\left|\gamma_{n}-\gamma_{n-1}\right|\left\|J_{r_{n-1}} x_{n-1}\right\| \\
& +\left|\delta_{n}-\delta_{n-1}\right|\left\|S_{n-1} x_{n-1}\right\| \\
& \left.+\delta_{n}\left\|S_{n} x_{n-1}-S_{n-1} x_{n-1}\right\|\right] \\
& +\frac{\left|\beta_{n}-\beta_{n-1}\right|}{\left(1-\beta_{n-1}\right)\left(1-\beta_{n}\right)} \\
& \times \| \alpha_{n-1} f\left(x_{n-1}\right)+\gamma_{n-1} J_{r_{n-1}} x_{n-1} \\
& +\delta_{n-1} S_{n-1} x_{n-1} \| \\
& \leq \frac{1}{1-\beta_{n}}\left[\left(\alpha_{n} \rho+\gamma_{n}\right)\left\|x_{n}-x_{n-1}\right\|\right. \\
& +\delta_{n}\left\|x_{n}-x_{n-1}\right\| \\
& +M\left(\left|\alpha_{n}-\alpha_{n-1}\right|+\left|\gamma_{n}-\gamma_{n-1}\right|\right. \\
& \left.+\left|\delta_{n}-\delta_{n-1}\right|+\left|r_{n-1}-r_{n}\right|\right) \\
& \left.+\delta_{n}\left\|S_{n} x_{n-1}-S_{n-1} x_{n-1}\right\|\right] \\
& +\frac{1}{\left(1-\beta_{n-1}\right)\left(1-\beta_{n}\right)}\left|\beta_{n}-\beta_{n-1}\right| M
\end{aligned}
$$




$$
\begin{aligned}
& =\frac{1}{1-\beta_{n}}\left[\left(1-\alpha_{n}-\beta_{n}+\alpha_{n} \rho\right) \| x_{n}-x_{n-1}||\right. \\
& +M\left(\left|\alpha_{n}-\alpha_{n-1}\right|+\left|\gamma_{n}-\gamma_{n-1}\right|\right. \\
& \left.\quad+\left|\delta_{n}-\delta_{n-1}\right|+\left|r_{n-1}-r_{n}\right|\right) \\
& \left.\quad+\delta_{n}|| S_{n} x_{n-1}-S_{n-1} x_{n-1} \|\right] \\
& +\frac{1}{\left(1-\beta_{n-1}\right)\left(1-\beta_{n}\right)}\left|\beta_{n}-\beta_{n-1}\right| M \\
& =\left(1-\frac{1-\rho}{1-\beta_{n}} \alpha_{n}\right)\left\|x_{n}-x_{n-1}\right\| \\
& +\frac{M}{1-\beta_{n}}\left(\left|\alpha_{n}-\alpha_{n-1}\right|+\left|\gamma_{n}-\gamma_{n-1}\right|\right. \\
& \left.\quad+\left|\delta_{n}-\delta_{n-1}\right|+\left|r_{n-1}-r_{n}\right|\right) \\
& \quad+\frac{\delta_{n}}{1-\beta_{n}}\left\|S_{n} x_{n-1}-S_{n-1} x_{n-1}\right\| \\
& +\frac{1}{\left(1-\beta_{n-1}\right)\left(1-\beta_{n}\right)}\left|\beta_{n}-\beta_{n-1}\right| M,
\end{aligned}
$$

where $\sup _{n \geq 0}\left\{M_{0}+\left\|f\left(x_{n}\right)\right\|+\left\|J_{r_{n}} x_{n}\right\|+\left\|S_{n} x_{n}\right\|\right\} \leq M$ for some $M>0$. Also, from (133) we have

$$
\begin{aligned}
x_{n+1}-x_{n}= & \sigma_{n}\left(y_{n}-y_{n-1}\right) \\
& +\left(\sigma_{n}-\sigma_{n-1}\right)\left(y_{n-1}-G y_{n-1}\right) \\
& +\left(1-\sigma_{n}\right)\left(G y_{n}-G y_{n-1}\right) .
\end{aligned}
$$

Taking into account condition (v), we may assume, without loss of generality, that $\left\{\beta_{n}\right\} \subset[a, b]$ for some $a, b \in(0,1)$. This together with (141) implies that

$$
\begin{aligned}
\left\|x_{n+1}-x_{n}\right\| \leq & \sigma_{n}\left\|y_{n}-y_{n-1}\right\| \\
& +\left|\sigma_{n}-\sigma_{n-1}\right|\left\|y_{n-1}-G y_{n-1}\right\| \\
& +\left(1-\sigma_{n}\right)\left\|G y_{n}-G y_{n-1}\right\| \\
\leq & \sigma_{n}\left\|y_{n}-y_{n-1}\right\| \\
& +\left|\sigma_{n}-\sigma_{n-1}\right|\left\|y_{n-1}-G y_{n-1}\right\| \\
& +\left(1-\sigma_{n}\right)\left\|y_{n}-y_{n-1}\right\| \\
= & \left\|y_{n}-y_{n-1}\right\| \\
& +\left|\sigma_{n}-\sigma_{n-1}\right|\left\|y_{n-1}-G y_{n-1}\right\| \\
\leq & \left(1-\frac{1-\rho}{1-\beta_{n}} \alpha_{n}\right)\left\|x_{n}-x_{n-1}\right\|
\end{aligned}
$$

$$
\begin{aligned}
& +\frac{M}{1-\beta_{n}}\left(\left|\alpha_{n}-\alpha_{n-1}\right|+\left|\gamma_{n}-\gamma_{n-1}\right|\right. \\
& \left.+\left|\delta_{n}-\delta_{n-1}\right|+\left|r_{n-1}-r_{n}\right|\right) \\
& +\frac{\delta_{n}}{1-\beta_{n}}\left\|S_{n} x_{n-1}-S_{n-1} x_{n-1}\right\| \\
& +\frac{1}{\left(1-\beta_{n-1}\right)\left(1-\beta_{n}\right)}\left|\beta_{n}-\beta_{n-1}\right| M \\
& +\left|\sigma_{n}-\sigma_{n-1}\right|\left\|y_{n-1}-G y_{n-1}\right\| \\
& \leq\left(1-\frac{1-\rho}{1-\beta_{n}} \alpha_{n}\right)\left\|x_{n}-x_{n-1}\right\| \\
& +M_{1}\left(\left|\alpha_{n}-\alpha_{n-1}\right|+\left|\gamma_{n}-\gamma_{n-1}\right|\right. \\
& \left.+\left|\delta_{n}-\delta_{n-1}\right|+\left|r_{n-1}-r_{n}\right|\right) \\
& +\left\|S_{n} x_{n-1}-S_{n-1} x_{n-1}\right\| \\
& +\left|\beta_{n}-\beta_{n-1}\right| M_{1}+\left|\sigma_{n}-\sigma_{n-1}\right| M_{1} \\
& =\left(1-\frac{1-\rho}{1-\beta_{n}} \alpha_{n}\right)\left\|x_{n}-x_{n-1}\right\| \\
& +M_{1}\left(\left|\sigma_{n}-\sigma_{n-1}\right|+\left|\alpha_{n}-\alpha_{n-1}\right|\right. \\
& +\left|\beta_{n}-\beta_{n-1}\right|+\left|\gamma_{n}-\gamma_{n-1}\right| \\
& \left.+\left|\delta_{n}-\delta_{n-1}\right|+\left|r_{n-1}-r_{n}\right|\right) \\
& +\left\|S_{n} x_{n-1}-S_{n-1} x_{n-1}\right\| \text {, }
\end{aligned}
$$

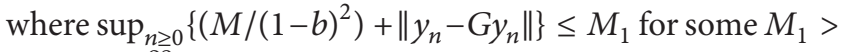
0 . Since $\sum_{n=0}^{\infty} \alpha_{n}=\infty$ and $\left((1-\rho) /\left(1-\beta_{n}\right)\right) \alpha_{n} \geq(1-\rho) \alpha_{n}$, we know that $\sum_{n=0}^{\infty}\left((1-\rho) /\left(1-\delta_{n}\right)\right) \alpha_{n}=\infty$. So, applying Lemma 9 to (143), we obtain from conditions (iii) and (iv) and the assumption on $\left\{S_{n}\right\}$ that

$$
\lim _{n \rightarrow \infty}\left\|x_{n+1}-x_{n}\right\|=0
$$

Next we show that $\left\|x_{n}-G x_{n}\right\| \rightarrow 0$ as $n \rightarrow \infty$. Indeed, according to Lemma 10 we have from (133)

$$
\begin{aligned}
\left\|y_{n}-p\right\|^{2}= & \| \alpha_{n}\left(f\left(x_{n}\right)-f(p)\right) \\
& +\beta_{n}\left(x_{n}-p\right)+\gamma_{n}\left(J_{r_{n}} x_{n}-p\right) \\
& +\delta_{n}\left(S_{n} x_{n}-p\right)+\alpha_{n}(f(p)-p) \|^{2} \\
\leq & \| \alpha_{n}\left(f\left(x_{n}\right)-f(p)\right)+\beta_{n}\left(x_{n}-p\right) \\
& +\gamma_{n}\left(J_{r_{n}} x_{n}-p\right)+\delta_{n}\left(S_{n} x_{n}-p\right) \|^{2} \\
& +2 \alpha_{n}\left\langle f(p)-p, J\left(y_{n}-p\right)\right\rangle
\end{aligned}
$$




$$
\begin{aligned}
\leq & \alpha_{n}\left\|f\left(x_{n}\right)-f(p)\right\|^{2}+\beta_{n}\left\|x_{n}-p\right\|^{2} \\
& +\gamma_{n}\left\|J_{r_{n}} x_{n}-p\right\|^{2}+\delta_{n}\left\|S_{n} x_{n}-p\right\|^{2} \\
& +2 \alpha_{n}\left\langle f(p)-p, J\left(y_{n}-p\right)\right\rangle \\
\leq & \alpha_{n} \rho\left\|x_{n}-p\right\|^{2}+\beta_{n}\left\|x_{n}-p\right\|^{2} \\
& +\gamma_{n}\left\|x_{n}-p\right\|^{2}+\delta_{n}\left\|x_{n}-p\right\|^{2} \\
& +2 \alpha_{n}\left\langle f(p)-p, J\left(y_{n}-p\right)\right\rangle \\
= & \left(\alpha_{n} \rho+\beta_{n}+\gamma_{n}+\delta_{n}\right)\left\|x_{n}-p\right\|^{2} \\
& +2 \alpha_{n}\left\langle f(p)-p, J\left(y_{n}-p\right)\right\rangle \\
\leq & \left(1-\alpha_{n}(1-\rho)\right)\left\|x_{n}-p\right\|^{2} \\
& +2 \alpha_{n}\left\langle f(p)-p, J\left(y_{n}-p\right)\right\rangle .
\end{aligned}
$$

Utilizing Lemma 17 we get from (133) and (145)

$$
\begin{aligned}
\left\|x_{n+1}-p\right\|^{2}= & \| \sigma_{n}\left(y_{n}-p\right) \\
& +\left(1-\sigma_{n}\right)\left(G y_{n}-p\right) \|^{2} \\
\leq & \sigma_{n}\left\|y_{n}-p\right\|^{2}+\left(1-\sigma_{n}\right)\left\|G y_{n}-p\right\|^{2} \\
& -\sigma_{n}\left(1-\sigma_{n}\right) g_{1}\left(\left\|y_{n}-G y_{n}\right\|\right) \\
\leq & \sigma_{n}\left\|y_{n}-p\right\|^{2}+\left(1-\sigma_{n}\right)\left\|y_{n}-p\right\|^{2} \\
& -\sigma_{n}\left(1-\sigma_{n}\right) g_{1}\left(\left\|y_{n}-G y_{n}\right\|\right) \\
= & \left\|y_{n}-p\right\|^{2}-\sigma_{n}\left(1-\sigma_{n}\right) g_{1}\left(\left\|y_{n}-G y_{n}\right\|\right) \\
\leq & \left(1-\alpha_{n}(1-\rho)\right)\left\|x_{n}-p\right\|^{2} \\
& +2 \alpha_{n}\left\langle f(p)-p, J\left(y_{n}-p\right)\right\rangle \\
& -\sigma_{n}\left(1-\sigma_{n}\right) g_{1}\left(\left\|y_{n}-G y_{n}\right\|\right) \\
\leq & \left\|x_{n}-p\right\|^{2}+2 \alpha_{n}\|f(p)-p\|\left\|y_{n}-p\right\| \\
& -\sigma_{n}\left(1-\sigma_{n}\right) g_{1}\left(\left\|y_{n}-G y_{n}\right\|\right),
\end{aligned}
$$

which hence yields

$$
\begin{aligned}
\sigma_{n}(1- & \left.\sigma_{n}\right) g_{1}\left(\left\|y_{n}-G y_{n}\right\|\right) \\
\leq & \left\|x_{n}-p\right\|^{2}-\left\|x_{n+1}-p\right\|^{2} \\
& +2 \alpha_{n}\|f(p)-p\|\left\|y_{n}-p\right\| \\
\leq & \left(\left\|x_{n}-p\right\|+\left\|x_{n+1}-p\right\|\right)\left\|x_{n}-x_{n+1}\right\| \\
& +2 \alpha_{n}\|f(p)-p\|\left\|y_{n}-p\right\| .
\end{aligned}
$$

Since $\alpha_{n} \rightarrow 0$ and $\left\|x_{n+1}-x_{n}\right\| \rightarrow 0$, from condition (v) and the boundedness of $\left\{x_{n}\right\}$ and $\left\{y_{n}\right\}$, it follows that

$$
\lim _{n \rightarrow \infty} g_{1}\left(\left\|y_{n}-G y_{n}\right\|\right)=0
$$

Utilizing the properties of $g_{1}$, we have

$$
\lim _{n \rightarrow \infty}\left\|y_{n}-G y_{n}\right\|=0
$$

Observe that

$$
\begin{aligned}
\left\|x_{n}-y_{n}\right\| \leq & \left\|x_{n}-x_{n+1}\right\| \\
& +\left\|x_{n+1}-y_{n}\right\|=\left\|x_{n}-x_{n+1}\right\| \\
& +\left(1-\sigma_{n}\right)\left\|G y_{n}-y_{n}\right\| \longrightarrow 0, \quad \text { as } n \longrightarrow \infty,
\end{aligned}
$$

and hence

$$
\begin{aligned}
\left\|x_{n}-G x_{n}\right\| \leq & \left\|x_{n}-y_{n}\right\| \\
& +\left\|y_{n}-G y_{n}\right\|+\left\|G y_{n}-G x_{n}\right\| \\
\leq & 2\left\|x_{n}-y_{n}\right\| \\
& +\left\|y_{n}-G y_{n}\right\| \longrightarrow 0, \quad \text { as } n \longrightarrow \infty .
\end{aligned}
$$

That is,

$$
\lim _{n \rightarrow \infty}\left\|x_{n}-y_{n}\right\|=0, \quad \lim _{n \rightarrow \infty}\left\|x_{n}-G x_{n}\right\|=0 .
$$

Next, let us show that

$$
\lim _{n \rightarrow \infty}\left\|J_{r_{n}} x_{n}-x_{n}\right\|=0, \quad \lim _{n \rightarrow \infty}\left\|S_{n} x_{n}-x_{n}\right\|=0 .
$$

Indeed, observe that $y_{n}$ can be rewritten as follows:

$$
\begin{aligned}
y_{n}= & \alpha_{n} f\left(x_{n}\right)+\beta_{n} x_{n} \\
& +\gamma_{n} J_{r_{n}} x_{n}+\delta_{n} S_{n} x_{n} \\
= & \alpha_{n} f\left(x_{n}\right)+\beta_{n} x_{n} \\
& +\left(\gamma_{n}+\delta_{n}\right) \frac{\gamma_{n} J_{r_{n}} x_{n}+\delta_{n} S_{n} x_{n}}{\gamma_{n}+\delta_{n}} \\
= & \alpha_{n} f\left(x_{n}\right)+\beta_{n} x_{n}+e_{n} \widehat{z}_{n},
\end{aligned}
$$


where $e_{n}=\gamma_{n}+\delta_{n}$ and $\widehat{z}_{n}=\left(\gamma_{n} J_{r_{n}} x_{n}+\delta_{n} S_{n} x_{n}\right) /\left(\gamma_{n}+\delta_{n}\right)$. Utilizing Lemma 13 and (154), we have

$$
\begin{aligned}
& \left\|y_{n}-p\right\|^{2}=\| \alpha_{n}\left(f\left(x_{n}\right)-p\right) \\
& +\beta_{n}\left(x_{n}-p\right)+e_{n}\left(\widehat{z}_{n}-p\right) \|^{2} \\
& \leq \alpha_{n}\left\|f\left(x_{n}\right)-p\right\|^{2}+\beta_{n}\left\|x_{n}-p\right\|^{2} \\
& +e_{n}\left\|\bar{z}_{n}-p\right\|^{2}-\beta_{n} e_{n} g_{3}\left(\left\|\widehat{z}_{n}-x_{n}\right\|\right) \\
& =\alpha_{n}\left\|f\left(x_{n}\right)-p\right\|^{2}+\beta_{n}\left\|x_{n}-p\right\|^{2} \\
& -\beta_{n} e_{n} g_{2}\left(\left\|\widehat{z}_{n}-x_{n}\right\|\right) \\
& +e_{n}\left\|\frac{\gamma_{n} J_{r_{n}} x_{n}+\delta_{n} S_{n} x_{n}}{\gamma_{n}+\delta_{n}}-p\right\|^{2} \\
& =\alpha_{n}\left\|f\left(x_{n}\right)-p\right\|^{2}+\beta_{n}\left\|x_{n}-p\right\|^{2} \\
& -\beta_{n} e_{n} g_{2}\left(\left\|\widehat{z}_{n}-x_{n}\right\|\right) \\
& +e_{n} \| \frac{\gamma_{n}}{\gamma_{n}+\delta_{n}}\left(J_{r_{n}} x_{n}-p\right) \\
& +\frac{\delta_{n}}{\gamma_{n}+\delta_{n}}\left(S_{n} x_{n}-p\right) \|^{2} \\
& \leq \alpha_{n}\left\|f\left(x_{n}\right)-p\right\|^{2}+\beta_{n}\left\|x_{n}-p\right\|^{2} \\
& -\beta_{n} e_{n} g_{2}\left(\left\|\widehat{z}_{n}-x_{n}\right\|\right) \\
& +e_{n}\left[\frac{\gamma_{n}}{\gamma_{n}+\delta_{n}}\left\|J_{r_{n}} x_{n}-p\right\|^{2}\right. \\
& \left.+\frac{\delta_{n}}{\gamma_{n}+\delta_{n}}\left\|S_{n} x_{n}-p\right\|^{2}\right] \\
& \leq \alpha_{n}\left\|f\left(x_{n}\right)-p\right\|^{2}+\beta_{n}\left\|x_{n}-p\right\|^{2} \\
& -\beta_{n} e_{n} g_{2}\left(\left\|\widehat{z}_{n}-x_{n}\right\|\right) \\
& +e_{n}\left[\frac{\gamma_{n}}{\gamma_{n}+\delta_{n}}\left\|x_{n}-p\right\|\right. \\
& \left.+\frac{\delta_{n}}{\gamma_{n}+\delta_{n}}\left\|x_{n}-p\right\|^{2}\right] \\
& =\alpha_{n}\left\|f\left(x_{n}\right)-p\right\|^{2} \\
& +\left(\beta_{n}+\gamma_{n}+\delta_{n}\right)\left\|x_{n}-p\right\|^{2} \\
& -\beta_{n} e_{n} g_{2}\left(\left\|\widehat{z}_{n}-x_{n}\right\|\right) \\
& \leq \alpha_{n}\left\|f\left(x_{n}\right)-p\right\|^{2}+\left\|x_{n}-p\right\|^{2} \\
& -\beta_{n} e_{n} g_{2}\left(\left\|\bar{z}_{n}-x_{n}\right\|\right),
\end{aligned}
$$

which hence implies that

$$
\begin{aligned}
\beta_{n} e_{n} g_{2}\left(\left\|\bar{z}_{n}-x_{n}\right\|\right) \leq & \alpha_{n}\left\|f\left(x_{n}\right)-p\right\|^{2} \\
& +\left\|x_{n}-p\right\|^{2}-\left\|y_{n}-p\right\|^{2} \\
\leq & \alpha_{n}\left\|f\left(x_{n}\right)-p\right\|^{2} \\
& +\left(\left\|x_{n}-p\right\|+\left\|y_{n}-p\right\|\right)\left\|x_{n}-y_{n}\right\| .
\end{aligned}
$$

Utilizing (152), conditions (i), (ii), and (v) and the boundedness of $\left\{x_{n}\right\},\left\{y_{n}\right\}$, and $\left\{f\left(x_{n}\right)\right\}$, we get

$$
\lim _{n \rightarrow \infty} g_{2}\left(\left\|\widehat{z}_{n}-x_{n}\right\|\right)=0 .
$$

From the properties of $g_{2}$, we have

$$
\lim _{n \rightarrow \infty}\left\|\widehat{z}_{n}-x_{n}\right\|=0 .
$$

Utilizing Lemma 17 and the definition of $\widehat{z}_{n}$, we have

$$
\begin{aligned}
\left\|\bar{z}_{n}-p\right\|^{2}= & \left\|\frac{\gamma_{n} J_{r_{n}} x_{n}+\delta_{n} S_{n} x_{n}}{\gamma_{n}+\delta_{n}}-p\right\|^{2} \\
= & \left\|\frac{\gamma_{n}}{\gamma_{n}+\delta_{n}}\left(J_{r_{n}} x_{n}-p\right)+\frac{\delta_{n}}{\gamma_{n}+\delta_{n}}\left(S_{n} x_{n}-p\right)\right\|^{2} \\
\leq & \frac{\gamma_{n}}{\gamma_{n}+\delta_{n}}\left\|J_{r_{n}} x_{n}-p\right\|^{2}+\frac{\delta_{n}}{\gamma_{n}+\delta_{n}}\left\|S_{n} x_{n}-p\right\|^{2} \\
& -\frac{\gamma_{n} \delta_{n}}{\left(\gamma_{n}+\delta_{n}\right)^{2}} g_{3}\left(\left\|J_{r_{n}} x_{n}-S_{n} x_{n}\right\|\right) \\
\leq & \frac{\gamma_{n}}{\gamma_{n}+\delta_{n}}\left\|x_{n}-p\right\|^{2}+\frac{\delta_{n}}{\gamma_{n}+\delta_{n}}\left\|x_{n}-p\right\|^{2} \\
& -\frac{\gamma_{n} \delta_{n}}{\left(\gamma_{n}+\delta_{n}\right)^{2}} g_{3}\left(\left\|J_{r_{n}} x_{n}-S_{n} x_{n}\right\|\right) \\
= & \left\|x_{n}-p\right\|^{2}-\frac{\gamma_{n} \delta_{n}}{\left(\gamma_{n}+\delta_{n}\right)^{2}} g_{3}\left(\left\|J_{r_{n}} x_{n}-S_{n} x_{n}\right\|\right),
\end{aligned}
$$

which leads to

$$
\begin{aligned}
& \frac{\gamma_{n} \delta_{n}}{\left(\gamma_{n}+\delta_{n}\right)^{2}} g_{3}\left(\left\|J_{r_{n}} x_{n}-S_{n} x_{n}\right\|\right) \\
& \quad \leq\left\|x_{n}-p\right\|^{2}-\left\|\widehat{z}_{n}-p\right\|^{2} \\
& \quad \leq\left(\left\|x_{n}-p\right\|+\left\|\bar{z}_{n}-p\right\|\right)\left\|x_{n}-\widehat{z}_{n}\right\| .
\end{aligned}
$$

Since $\left\{x_{n}\right\}$ and $\left\{\widehat{z}_{n}\right\}$ are bounded and $\left\|\widehat{z}_{n}-x_{n}\right\| \rightarrow 0$ as $n \rightarrow$ $\infty$, we deduce from condition (ii) that

$$
\lim _{n \rightarrow \infty} g_{3}\left(\left\|J_{r_{n}} x_{n}-S_{n} x_{n}\right\|\right)=0 .
$$

From the properties of $g_{3}$, we have

$$
\lim _{n \rightarrow \infty}\left\|J_{r_{n}} x_{n}-S_{n} x_{n}\right\|=0 .
$$


On the other hand, $y_{n}$ can also be rewritten as follows:

$$
\begin{aligned}
y_{n}= & \alpha_{n} f\left(x_{n}\right)+\beta_{n} x_{n} \\
& +\gamma_{n} J_{r_{n}} x_{n}+\delta_{n} S_{n} x_{n} \\
= & \beta_{n} x_{n}+\gamma_{n} J_{r_{n}} x_{n} \\
& +\left(\alpha_{n}+\delta_{n}\right) \frac{\alpha_{n} f\left(x_{n}\right)+\delta_{n} S_{n} x_{n}}{\alpha_{n}+\delta_{n}} \\
= & \beta_{n} x_{n}+\gamma_{n} J_{r_{n}} x_{n}+d_{n} \widetilde{z}_{n},
\end{aligned}
$$

where $d_{n}=\alpha_{n}+\delta_{n}$ and $\widetilde{z}_{n}=\left(\alpha_{n} f\left(x_{n}\right)+\delta_{n} S_{n} x_{n}\right) /\left(\alpha_{n}+\delta_{n}\right)$. Utilizing Lemma 13 and the convexity of $\|\cdot\|^{2}$, we have

$$
\begin{aligned}
& \left\|y_{n}-p\right\|^{2}=\| \beta_{n}\left(x_{n}-p\right) \\
& +\gamma_{n}\left(J_{r_{n}} x_{n}-p\right)+d_{n}\left(\widetilde{z}_{n}-p\right) \|^{2} \\
& \leq \beta_{n}\left\|x_{n}-p\right\|^{2}+\gamma_{n}\left\|J_{r_{n}} x_{n}-p\right\|^{2} \\
& +d_{n}\left\|\tilde{z}_{n}-p\right\|^{2}-\beta_{n} \gamma_{n} g_{4}\left(\left\|x_{n}-J_{r_{n}} x_{n}\right\|\right) \\
& =\beta_{n}\left\|x_{n}-p\right\|^{2}+\gamma_{n}\left\|J_{r_{n}} x_{n}-p\right\|^{2} \\
& +d_{n}\left\|\frac{\alpha_{n} f\left(x_{n}\right)+\delta_{n} S_{n} x_{n}}{\alpha_{n}+\delta_{n}}-p\right\|^{2} \\
& -\beta_{n} \gamma_{n} g_{4}\left(\left\|x_{n}-J_{r_{n}} x_{n}\right\|\right) \\
& =\beta_{n}\left\|x_{n}-p\right\|^{2}+\gamma_{n}\left\|J_{r_{n}} x_{n}-p\right\|^{2} \\
& +d_{n} \| \frac{\alpha_{n}}{\alpha_{n}+\delta_{n}}\left(f\left(x_{n}\right)-p\right) \\
& +\frac{\delta_{n}}{\alpha_{n}+\delta_{n}}\left(S_{n} x_{n}-p\right) \|^{2} \\
& -\beta_{n} \gamma_{n} g_{4}\left(\left\|x_{n}-J_{r_{n}} x_{n}\right\|\right) \\
& \leq \beta_{n}\left\|x_{n}-p\right\|^{2}+\gamma_{n}\left\|x_{n}-p\right\|^{2} \\
& +d_{n}\left[\frac{\alpha_{n}}{\alpha_{n}+\delta_{n}}\left\|f\left(x_{n}\right)-p\right\|^{2}\right. \\
& \left.+\frac{\delta_{n}}{\alpha_{n}+\delta_{n}}\left\|S_{n} x_{n}-p\right\|^{2}\right] \\
& -\beta_{n} \gamma_{n} g_{4}\left(\left\|x_{n}-J_{r_{n}} x_{n}\right\|\right) \\
& \leq \alpha_{n}\left\|f\left(x_{n}\right)-p\right\|^{2} \\
& +\left(\beta_{n}+\gamma_{n}+\delta_{n}\right)\left\|x_{n}-p\right\|^{2} \\
& -\beta_{n} \gamma_{n} g_{4}\left(\left\|x_{n}-J_{r_{n}} x_{n}\right\|\right) \\
& \leq \alpha_{n}\left\|f\left(x_{n}\right)-p\right\|^{2}+\left\|x_{n}-p\right\|^{2} \\
& -\beta_{n} \gamma_{n} g_{4}\left(\left\|x_{n}-J_{r_{n}} x_{n}\right\|\right) \text {, }
\end{aligned}
$$

which hence implies that

$$
\begin{aligned}
\beta_{n} \gamma_{n} g_{4}\left(\left\|x_{n}-J_{r_{n}} x_{n}\right\|\right) \leq & \alpha_{n}\left\|f\left(x_{n}\right)-p\right\|^{2} \\
& +\left\|x_{n}-p\right\|^{2}-\left\|y_{n}-p\right\|^{2} \\
\leq & \alpha_{n}\left\|f\left(x_{n}\right)-p\right\|^{2} \\
& +\left(\left\|x_{n}-p\right\|+\left\|y_{n}-p\right\|\right)\left\|x_{n}-y_{n}\right\| .
\end{aligned}
$$

From (152), conditions (i), (ii), and (v), and the boundedness of $\left\{x_{n}\right\},\left\{y_{n}\right\}$, and $\left\{f\left(x_{n}\right)\right\}$, we have

$$
\lim _{n \rightarrow \infty} g_{4}\left(\left\|x_{n}-J_{r_{n}} x_{n}\right\|\right)=0 \text {. }
$$

Utilizing the properties of $g_{4}$, we have

$$
\lim _{n \rightarrow \infty}\left\|x_{n}-J_{r_{n}} x_{n}\right\|=0 .
$$

Note that

$$
\left\|x_{n}-S_{n} x_{n}\right\| \leq\left\|x_{n}-J_{r_{n}} x_{n}\right\|+\left\|J_{r_{n}} x_{n}-S_{n} x_{n}\right\| .
$$

From (162) and (167), we get

$$
\lim _{n \rightarrow \infty}\left\|x_{n}-S_{n} x_{n}\right\|=0
$$

In terms of (169) and Lemma 14, we have

$$
\begin{aligned}
\left\|x_{n}-S x_{n}\right\| \leq & \left\|x_{n}-S_{n} x_{n}\right\| \\
& +\left\|S_{n} x_{n}-S x_{n}\right\| \longrightarrow \text { as } n \longrightarrow \infty .
\end{aligned}
$$

That is,

$$
\lim _{n \rightarrow \infty}\left\|x_{n}-S x_{n}\right\|=0 \text {. }
$$

Furthermore, repeating the same arguments as those of (104) in the proof of Theorem 23, we can conclude that

$$
\lim _{n \rightarrow \infty}\left\|x_{n}-J_{r} x_{n}\right\|=0
$$

for a fixed number $r$ such that $\varepsilon>r>0$.

Define a mapping $W x=\left(1-\theta_{1}-\theta_{2}\right) J_{r} x+\theta_{1} S x+\theta_{2} G x$, where $\theta_{1}, \theta_{2} \in(0,1)$ are two constants with $\theta_{1}+\theta_{2}<1$. Then by Lemma 16, we have that $\operatorname{Fix}(W)=\operatorname{Fix}\left(J_{r}\right) \cap \operatorname{Fix}(S) \cap$ $\operatorname{Fix}(G)=F$. We observe that

$$
\begin{aligned}
\left\|x_{n}-W x_{n}\right\|= & \|\left(1-\theta_{1}-\theta_{2}\right)\left(x_{n}-J_{r} x_{n}\right) \\
& +\theta_{1}\left(x_{n}-S x_{n}\right)+\theta_{2}\left(x_{n}-G x_{n}\right) \| \\
\leq & \left(1-\theta_{1}-\theta_{2}\right)\left\|x_{n}-J_{r} x_{n}\right\| \\
& +\theta_{1}\left\|x_{n}-S x_{n}\right\|+\theta_{2}\left\|x_{n}-G x_{n}\right\| .
\end{aligned}
$$

From (152), (171), and (172), we obtain

$$
\lim _{n \rightarrow \infty}\left\|x_{n}-W x_{n}\right\|=0
$$


Repeating the same arguments as those of (107) in the proof of Theorem 23, we can deduce that

$$
\limsup _{n \rightarrow \infty}\left\langle f(q)-q, J\left(x_{n}-q\right)\right\rangle \leq 0 .
$$

Since $J$ is norm-to-weak ${ }^{*}$ uniformly continuous over bounded subsets of $X$, we obtain from $\left\|x_{n}-y_{n}\right\| \rightarrow 0$ that

$$
\limsup _{n \rightarrow \infty}\left\langle f(q)-q, J\left(y_{n}-q\right)\right\rangle \leq 0 .
$$

Finally, let us show that $x_{n} \rightarrow q$ as $n \rightarrow \infty$. Indeed, observe that

$$
\begin{aligned}
\left\|y_{n}-q\right\|^{2}= & \| \alpha_{n}\left(f\left(x_{n}\right)-f(q)\right) \\
& +\beta_{n}\left(x_{n}-q\right)+\gamma_{n}\left(J_{r_{n}} x_{n}-q\right) \\
& +\delta_{n}\left(S_{n} x_{n}-q\right)+\alpha_{n}(f(q)-q) \|^{2} \\
\leq & \| \alpha_{n}\left(f\left(x_{n}\right)-f(q)\right)+\beta_{n}\left(x_{n}-q\right) \\
& +\gamma_{n}\left(J_{r_{n}} x_{n}-q\right)+\delta_{n}\left(S_{n} x_{n}-q\right) \|^{2} \\
& +2 \alpha_{n}\left\langle f(q)-q, J\left(y_{n}-q\right)\right\rangle \\
\leq & \alpha_{n}\left\|f\left(x_{n}\right)-f(q)\right\|^{2}+\beta_{n}\left\|x_{n}-q\right\|^{2} \\
& +\gamma_{n}\left\|J_{r_{n}} x_{n}-q\right\|^{2}+\delta_{n}\left\|S_{n} x_{n}-q\right\|^{2} \\
& +2 \alpha_{n}\left\langle f(q)-q, J\left(y_{n}-q\right)\right\rangle \\
\leq & \alpha_{n} \rho\left\|x_{n}-q\right\|^{2}+\beta_{n}\left\|x_{n}-q\right\|^{2} \\
& +\gamma_{n}\left\|x_{n}-q\right\|+\delta_{n}\left\|x_{n}-q\right\|^{2} \\
& +2 \alpha_{n}\left\langle f(q)-q, J\left(y_{n}-q\right)\right\rangle \\
= & \left(\alpha_{n} \rho+\beta_{n}+\gamma_{n}+\delta_{n}\right)\left\|x_{n}-q\right\|^{2} \\
& +2 \alpha_{n}\left\langle f(q)-q, J\left(y_{n}-q\right)\right\rangle \\
= & \left(1-\alpha_{n}(1-\rho)\right)\left\|x_{n}-q\right\|^{2} \\
& +2 \alpha_{n}\left\langle f(q)-q, J\left(y_{n}-q\right)\right\rangle, \\
&
\end{aligned}
$$

and hence

$$
\begin{aligned}
\left\|x_{n+1}-q\right\|^{2} \leq & \sigma_{n}\left\|y_{n}-q\right\|^{2} \\
& +\left(1-\sigma_{n}\right)\left\|G y_{n}-q\right\|^{2} \\
\leq & \left\|y_{n}-q\right\|^{2} \\
\leq & \left(1-\alpha_{n}(1-\rho)\right)\left\|x_{n}-q\right\|^{2} \\
& +2 \alpha_{n}\left\langle f(q)-q, J\left(y_{n}-q\right)\right\rangle .
\end{aligned}
$$

Applying Lemma 9 to (178), we conclude from condition (i) and (178) that $x_{n} \rightarrow q$ as $n \rightarrow \infty$. This completes the proof.
The following results can be obtained from Theorem 28 . We, therefore, omit the proof.

Corollary 29. Under the same conditions as those in Corollary 24 , let $\left\{x_{n}\right\}$ be the sequence generated from any given $x_{0} \in C$ by

$$
\begin{array}{rl}
y_{n}=\alpha_{n} & f\left(x_{n}\right)+\beta_{n} x_{n}+\gamma_{n} J_{r_{n}} x_{n}+\delta_{n} S x_{n}, \\
x_{n+1}= & \sigma_{n} y_{n}+\left(1-\sigma_{n}\right) \Pi_{C}\left(I-\mu_{1} B_{1}\right) \\
& \times \Pi_{C}\left(I-\mu_{2} B_{2}\right) y_{n}, \quad \forall n \geq 0 .
\end{array}
$$

Then $\left\{x_{n}\right\}$ converges strongly to $q \in F=\operatorname{Fix}(S) \cap \Omega \cap A^{-1} 0$, which solves the following VIP:

$$
\langle q-f(q), J(q-p)\rangle \leq 0, \quad \forall p \in F .
$$

Corollary 30. Under the same conditions as those in Corollary 25 , let $\left\{x_{n}\right\}$ be the sequence generated from any given $x_{0} \in C$ by

$$
\begin{gathered}
y_{n}=\alpha_{n} f\left(x_{n}\right)+\beta_{n} x_{n}+\gamma_{n} J_{r_{n}} x_{n}+\delta_{n} S_{n} x_{n}, \\
x_{n+1}=\sigma_{n} y_{n}+\left(1-\sigma_{n}\right)((1-\alpha) I+\alpha V) y_{n}, \quad \forall n \geq 0 .
\end{gathered}
$$

Then $\left\{x_{n}\right\}$ converges strongly to $q \in F=\bigcap_{i=0}^{\infty} \operatorname{Fix}\left(S_{i}\right) \cap \operatorname{Fix}(V) \cap$ $A^{-1} 0$, which solves the following VIP:

$$
\langle q-f(q), J(q-p)\rangle \leq 0, \quad \forall p \in F .
$$

Corollary 31. Under the same conditions as those in Corollary 26 , let $\left\{x_{n}\right\}$ be the sequence generated from any given $x_{0} \in C$ by

$$
\begin{gathered}
y_{n}=\alpha_{n} f\left(x_{n}\right)+\beta_{n} x_{n}+\gamma_{n} J_{r_{n}} x_{n}+\delta_{n} S x_{n}, \\
x_{n+1}=\sigma_{n} y_{n}+\left(1-\sigma_{n}\right)((1-\alpha) I+\alpha V) y_{n}, \quad \forall n \geq 0 .
\end{gathered}
$$

Then $\left\{x_{n}\right\}$ converges strongly to $q \in F=\operatorname{Fix}(S) \cap \operatorname{Fix}(V) \cap$ $A^{-1} 0$, which solves the following VIP:

$$
\langle q-f(q), J(q-p)\rangle \leq 0, \quad \forall p \in F .
$$

Remark 32. Our Theorem 28 improves, extends, supplements, and develops Cai and $\mathrm{Bu}$ [10, Theorem 3.1 and Corollary 3.2] and Jung [5, Theorems 3.1] in the following aspects.

(i) The problem of finding a point $q \in \bigcap_{n} \operatorname{Fix}\left(S_{n}\right) \cap \Omega \cap$ $A^{-1} 0$ in our Theorem 28 is more general and more subtle than every one of both the problem of finding a point $q \epsilon$ $\bigcap_{n} \operatorname{Fix}\left(S_{n}\right) \cap \Omega$ in Cai and $\mathrm{Bu}[10$, Theorem 3.1] and the problem of finding a point $q \in A^{-1} 0$ in Jung [5, Theorem 3.1].

(ii) Our Theorem 28 drops the assumption of the asymptotical regularity of $\left\{x_{n}\right\}$ in [5, Theorems 3.1] (i.e., $\lim _{n \rightarrow \infty}\left\|x_{n}-x_{n+1}\right\|=0$ ).

(iii) Cai and Bu's proof in [10, Theorem 3.1] depends on the argument techniques in [13], inequality (21) in 2-uniformly smooth Banach spaces, and inequality (27) in smooth and uniform convex Banach spaces. Jung's proof in 
[5, Theorem 3.1] depends on the resolvent identity in Proposition 1. It is worth emphasizing that the proof of our Theorem 28 does not depend on the argument techniques in [13], inequality (21) in 2-uniformly smooth Banach spaces, and inequality (27) in smooth and uniform convex Banach spaces. However, it depends on the resolvent identity in Proposition 1 and the inequalities in uniform convex Banach spaces; see Lemmas 13 and 17 in Section 2 of this paper.

(iv) The assumption of the uniformly convex and 2uniformly smooth Banach space $X$ in [10, Theorem 3.1] is weakened to the one of the uniformly convex Banach space $X$ having a uniformly Gateaux differentiable norm in our Theorem 28.

(v) The iterative scheme in our Theorem 28 is very different from every one in both [10, Theorem 3.1] and [5, Theorem 3.1] because the first iteration step in the iterative scheme of [10, Theorem 3.1] is given by $y_{n}=\alpha_{n} f\left(x_{n}\right)+$ $\left(1-\alpha_{n}\right) \Pi_{C}\left(I-\mu_{1} B_{1}\right) \Pi_{C}\left(I-\mu_{2} B_{2}\right) x_{n}$ and the first iteration step in the iterative scheme of [5, Theorem 3.1] is given by $y_{n}=\alpha_{n} f\left(x_{n}\right)+\left(1-\alpha_{n}\right) J_{r_{n}} x_{n}$. In the meantime, it is clear that the second iteration steps for $x_{n+1}$ in three iterative schemes are completely different.

(vi) The problem of finding a point $q \in \operatorname{Fix}(S) \cap \Omega$ in $[10$, Corollary 3.2] is extended to develop the problem of finding a point $q \in \operatorname{Fix}(S) \cap \Omega \cap A^{-1} 0$ in our Corollary 29 .

\section{Acknowledgments}

This research was partially supported by the National Science Foundation of China (11071169), Innovation Program of Shanghai Municipal Education Commission (09ZZ133), and Ph.D. Program Foundation of Ministry of Education of China (20123127110002). This research was partially supported by a Grant from NSC 102-2115-M-037-001.

\section{References}

[1] L.-C. Ceng, Q. H. Ansari, and J.-C. Yao, "Mann-type steepestdescent and modified hybrid steepest-descent methods for variational inequalities in Banach spaces," Numerical Functional Analysis and Optimization, vol. 29, no. 9-10, pp. 987-1033, 2008.

[2] V. Barbu, Nonlinear Semigroups and Differential Equations in Banach Spaces, Noordhoff, Leiden, The Netherlands, 1976.

[3] K. Aoyama, Y. Kimura, W. Takahashi, and M. Toyoda, "Approximation of common fixed points of a countable family of nonexpansive mappings in a Banach space," Nonlinear Analysis. Theory, Methods \& Applications A, vol. 67, no. 8, pp. 2350-2360, 2007.

[4] L.-C. Ceng, A. R. Khan, Q. H. Ansari, and J.-C. Yao, "Strong convergence of composite iterative schemes for zeros of $m$-accretive operators in Banach spaces," Nonlinear Analysis. Theory, Methods \& Applications A, vol. 70, no. 5, pp. 1830-1840, 2009.

[5] J. S. Jung, "Convergence of composite iterative methods for finding zeros of accretive operators," Nonlinear Analysis. Theory, Methods \& Applications A, vol. 71, no. 5-6, pp. 1736-1746, 2009.

[6] L. C. Zeng, G. M. Lee, and N. C. Wong, "Ishikawa iteration with errors for approximating fixed points of strictly pseudocontractive mappings of Browder-Petryshyn type," Taiwanese Journal of Mathematics, vol. 10, no. 1, pp. 87-99, 2006.
[7] H. Iiduka and W. Takahashi, "Strong convergence theorems for nonexpansive mappings and inverse-strongly monotone mappings," Nonlinear Analysis. Theory, Methods \& Applications A, vol. 61, no. 3, pp. 341-350, 2005.

[8] W. Takahashi and M. Toyoda, "Weak convergence theorems for nonexpansive mappings and monotone mappings," Journal of Optimization Theory and Applications, vol. 118, no. 2, pp. 417428, 2003.

[9] H. Iiduka, W. Takahashi, and M. Toyoda, "Approximation of solutions of variational inequalities for monotone mappings," Panamerican Mathematical Journal, vol. 14, no. 2, pp. 49-61, 2004.

[10] G. Cai and S. Bu, "Convergence analysis for variational inequality problems and fixed point problems in 2-uniformly smooth and uniformly convex Banach spaces," Mathematical and Computer Modelling, vol. 55, no. 3-4, pp. 538-546, 2012.

[11] R. U. Verma, "Projection methods, algorithms, and a new system of nonlinear variational inequalities," Computers \& Mathematics with Applications, vol. 41, no. 7-8, pp. 1025-1031, 2001.

[12] J.-L. Lions and G. Stampacchia, "Variational inequalities," Communications on Pure and Applied Mathematics, vol. 20, pp. 493519, 1967.

[13] L.-C. Ceng, C.-Y. Wang, and J.-C. Yao, "Strong convergence theorems by a relaxed extragradient method for a general system of variational inequalities," Mathematical Methods of Operations Research, vol. 67, no. 3, pp. 375-390, 2008.

[14] G. M. Korpelevič, "An extragradient method for finding saddle points and for other problems," Ékonomika i Matematicheskie Metody, vol. 12, no. 4, pp. 747-756, 1976.

[15] J. S. Jung, "Iterative approaches to common fixed points of nonexpansive mappings in Banach spaces," Journal of Mathematical Analysis and Applications, vol. 302, no. 2, pp. 509-520, 2005.

[16] Y. Yao, Y.-C. Liou, S. M. Kang, and Y. Yu, "Algorithms with strong convergence for a system of nonlinear variational inequalities in Banach spaces," Nonlinear Analysis. Theory, Methods \& Applications A, vol. 74, no. 17, pp. 6024-6034, 2011.

[17] Y. Censor, A. Gibali, and S. Reich, "The subgradient extragradient method for solving variational inequalities in Hilbert space," Journal of Optimization Theory and Applications, vol. 148, no. 2, pp. 318-335, 2011.

[18] L.-C. Ceng, Q. H. Ansari, and J.-C. Yao, "Relaxed extragradient methods for finding minimum-norm solutions of the split feasibility problem," Nonlinear Analysis. Theory, Methods \& Applications A, vol. 75, no. 4, pp. 2116-2125, 2012.

[19] L.-C. Ceng, Q. H. Ansari, and J.-C. Yao, "Relaxed extragradient iterative methods for variational inequalities," Applied Mathematics and Computation, vol. 218, no. 3, pp. 1112-1123, 2011.

[20] L.-C. Ceng, Q. H. Ansari, and J.-C. Yao, "An extragradient method for solving split feasibility and fixed point problems," Computers \& Mathematics with Applications, vol. 64, no. 4, pp. 633-642, 2012.

[21] L.-C. Ceng, Q. H. Ansari, N.-C. Wong, and J.-C. Yao, "An extragradient-like approximation method for variational inequalities and fixed point problems," Fixed Point Theory and Applications, vol. 2011, article 22, 22 pages, 2011.

[22] L.-C. Ceng, N. Hadjisavvas, and N.-C. Wong, "Strong convergence theorem by a hybrid extragradient-like approximation method for variational inequalities and fixed point problems," Journal of Global Optimization, vol. 46, no. 4, pp. 635-646, 2010.

[23] L.-C. Ceng, S.-M. Guu, and J.-C. Yao, "Finding common solutions of a variational inequality, a general system of variational 
inequalities, and a fixed-point problem via a hybrid extragradient method," Fixed Point Theory and Applications, vol. 2011, Article ID 10.1155/2011/626159, 22 pages, 2011.

[24] K. Aoyama, H. Iiduka, and W. Takahashi, "Weak convergence of an iterative sequence for accretive operators in Banach spaces," Fixed Point Theory and Applications, vol. 2006, Article ID 35390, 13 pages, 2006.

[25] L. C. Ceng and A. Petruşel, "Krasnoselski-Mann iterations for hierarchical fixed point problems for a finite family of nonself mappings in Banach spaces," Journal of Optimization Theory and Applications, vol. 146, no. 3, pp. 617-639, 2010.

[26] S. Kamimura and W. Takahashi, "Weak and strong convergence of solutions to accretive operator inclusions and applications," Set-Valued Analysis, vol. 8, no. 4, pp. 361-374, 2000.

[27] H. K. Xu, "Inequalities in Banach spaces with applications," Nonlinear Analysis. Theory, Methods \& Applications A, vol. 16, no. 12, pp. 1127-1138, 1991.

[28] S. Kamimura and W. Takahashi, "Strong convergence of a proximal-type algorithm in a Banach space," SIAM Journal on Optimization, vol. 13, no. 3, pp. 938-945, 2002.

[29] Y. Takahashi, K. Hashimoto, and M. Kato, "On sharp uniform convexity, smoothness, and strong type, cotype inequalities," Journal of Nonlinear and Convex Analysis, vol. 3, no. 2, pp. 267281, 2002.

[30] H.-K. Xu, "Iterative algorithms for nonlinear operators," Journal of the London Mathematical Society, vol. 66, no. 1, pp. 240-256, 2002.

[31] S. Reich, "Weak convergence theorems for nonexpansive mappings in Banach spaces," Journal of Mathematical Analysis and Applications, vol. 67, no. 2, pp. 274-276, 1979.

[32] Y. J. Cho, H. Zhou, and G. Guo, "Weak and strong convergence theorems for three-step iterations with errors for asymptotically nonexpansive mappings," Computers \& Mathematics with Applications, vol. 47, no. 4-5, pp. 707-717, 2004.

[33] K. Aoyama, Y. Kimura, W. Takahashi, and M. Toyoda, "Approximation of common fixed points of a countable family of nonexpansive mappings in a Banach space," Nonlinear Analysis. Theory, Methods \& Applications A, vol. 67, no. 8, pp. 2350-2360, 2007.

[34] R. E. Bruck Jr., "Properties of fixed-point sets of nonexpansive mappings in Banach spaces," Transactions of the American Mathematical Society, vol. 179, pp. 251-262, 1973. 


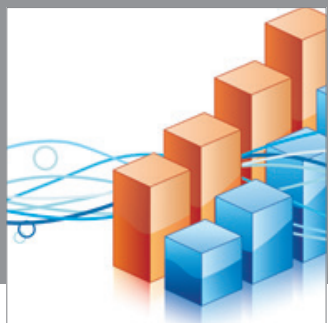

Advances in

Operations Research

mansans

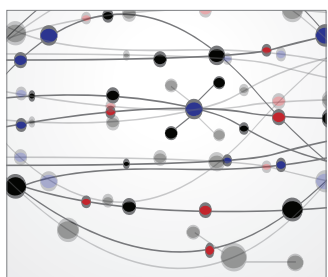

The Scientific World Journal
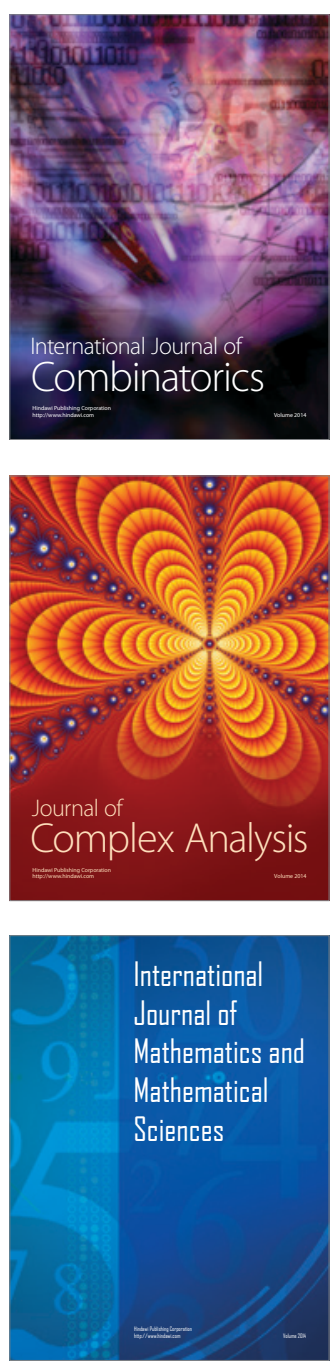
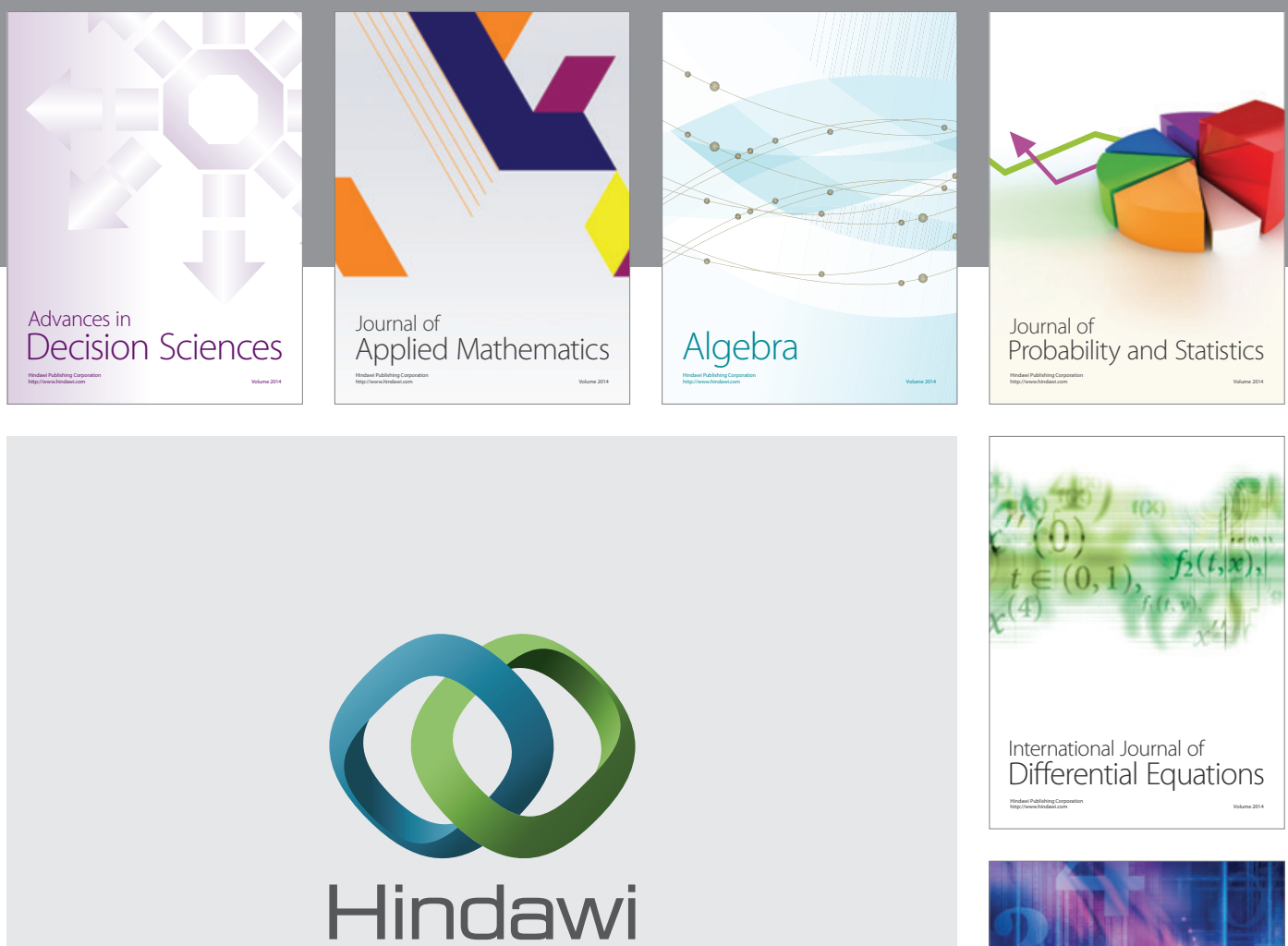

Submit your manuscripts at http://www.hindawi.com
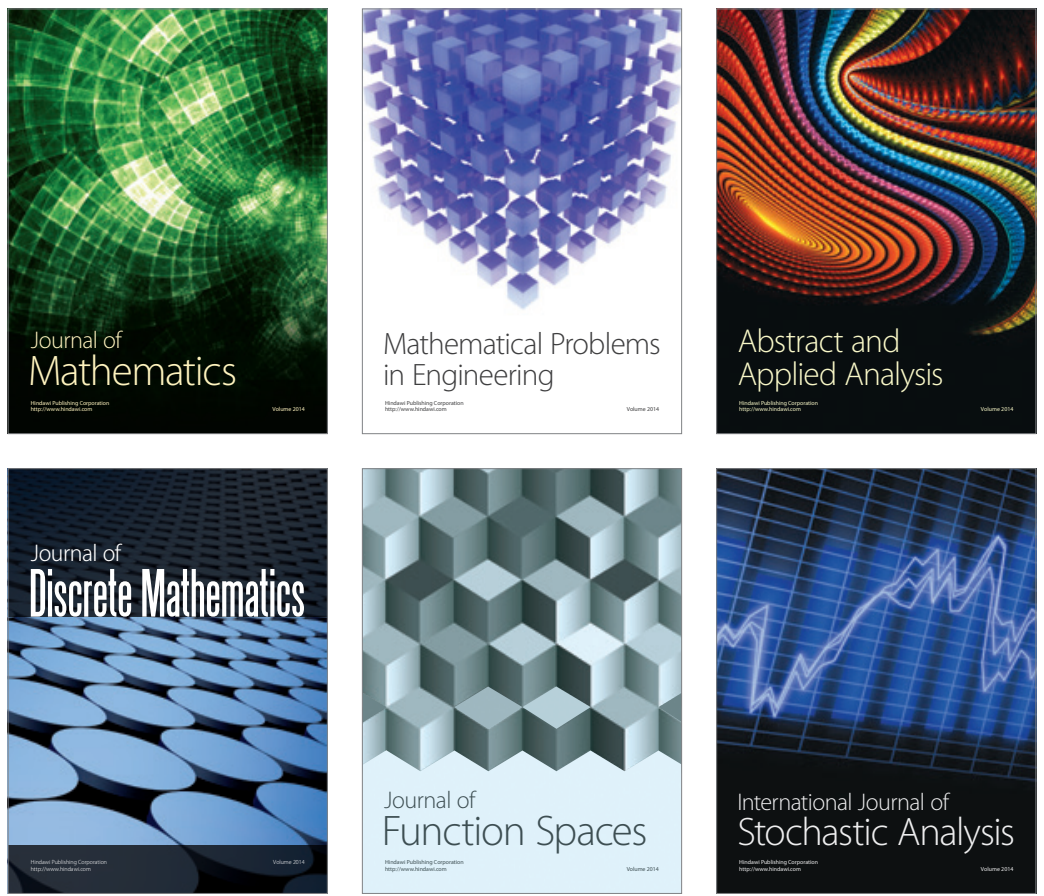

Journal of

Function Spaces

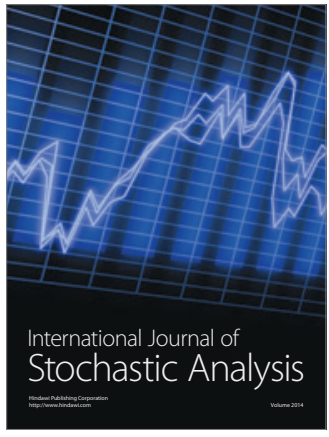

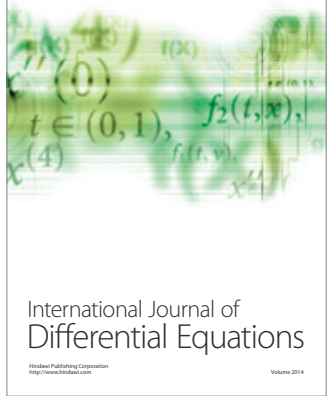
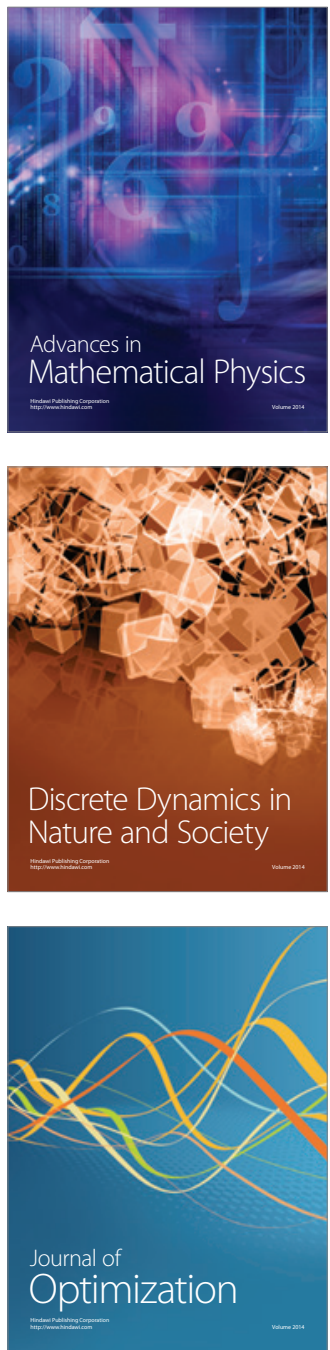